November 24,1993

Ms. Lynn A. Kszos, Aquatic Toxicologist

Environmental Sciences Division

Oak Ridge National Laboratory

P. O. Box 2008, MS -6351

Oak Ridge, Tennessee 37831-6351

Dear Lynn:

Enclosed is a copy of the report presenting results of TVA toxicity testing using fathead minnows only (per correspondence, letter $\mathrm{J}$. Moses to $\mathrm{T}$. $\mathrm{L}$. Phipps, October 14, 1993) during the Clinch River - Environmental Restoration Program (CR-ERP) Study, October 21-28, 1993. Attachments to the report inclyde Chain of Custody Forms - Originals (I), Toxicity Test Bench Sheets and Statstical Analyses (II), and Reference Toxicant Test Information (III). Exposure of fathead minnows to water column samples collected from Poplar Creek Mile 2.9, Mile 4.3, and Mile 5.1 on October 20,22 , and 25 resulted in no toxicity to test organisms.

Please call me (205-729-3340) if you have questions or comments regarding the report or conductance of the study.

Sincerely,

Jennifer Moses, Unit Supervisor

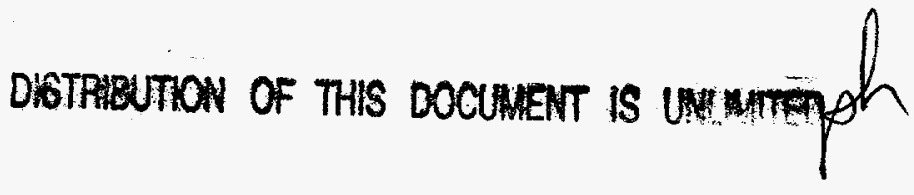

JY:DTH

Enclosures

cc: H. C. Jones, HB 2C-C

J. D. Hilligan, HB 2C-C (w/enclosures)

D. C. Wade, OSA 1B-M (w/enclosures) Files, WM, HB 2C-C (w/enclosures)

Prepared by Jennifer Moses

PLARC501-1950

APPROVED FOR RELEASE OR

PUBLICATION. INTEL. PROP. GP, OFC. OF CHIEF COUNSEL, DOE/ORO

By E\& \& low Date $11 / 21197$ 


\section{DISCLAIMER}

This report was prepared as an account of work sponsored by an agency of the United States Government. Neither the United States Government nor any agency thereof, nor any of their employees, make any warranty, express or implied, or assumes any legal liability or responsibility for the accuracy, completeness, or usefulness of any information, apparatus, product, or process disclosed, or represents that its use would not infringe privately owned rights. Reference herein to any specific commercial product, process, or service by trade name, trademark, manufacturer, or otherwise does not necessarily constitute or imply its endorsement, recommendation, or favoring by the United States Government or any agency thereof. The views and opinions of authors expressed herein do not necessarily state or reflect those of the United States Government or any agency thereof. 


\section{DISCLAIMER}

Portions of this document may be illegible electronic image products. Images are produced from the best available original document. 
STANDARD REPORT FORM

\section{STATIC RENEWAL TESTS USING \\ PIMEPHALES PROMELAS (FATHEAD MINNOWS)}

Test Title: Clinch River - Environmental Restoration Program (CR-ERP)

Study, Ambient Water Toxicity

Principal Investigator: Damien J. Simbeck

Starting Date: October 21, 1993

Ending Date: October 28, 1993

\section{$1.0 \quad$ EXECUTIVE SUMMARY}

Clinch River - Environmental Restoration Program (CR-ERP) personnel and Tennessee Valley Authority (TVA) personnel conducted a study during the week of october 21-28, 1993, as described in the statement of Work (SOW) document. [1] The organisms specified for testing were larval fathead minnows, Pimephales promelas, and the daphnid, Ceriodaphnia dubia. Due to serious reproduction/embryo abortion problems with the TVA daphnid cultures, TVA conducted tests during this study period using only fathead minnows. A split sample test using daphnids only will be scheduled during 1994 as a substitute for this study period (see letter, J. Moses to T. L. Phipps, October 14, 1993). [2]

Surface water samples were collected by TVA Field Engineering personnel from Poplar Creek Mile 2.9, Mile 4.3, and Mile 5.1 on October 20, 22, and 25. Samples were split and provided to the CR-ERP and TVA toxicology laboratories for testing. Exposure of test organisms to these samples resulted in no toxicity (survival or growth) in testing conducted by TVA.

\subsection{SAMPLE COLLECTION/TREATMENTS}

2.1 Test Sample Identification (Chemical/Effluent/Elutriate, etc.): Samples were collected for biomonitoring at the following three sites: Poplar Creek Mile 2.9, Mile 4.3 and Mile 5.1.

2.2 Control and/or Dilution Water: Laboratory culture medium consisting of moderately hard reconstituted water was used as control and dilution water for toxicity tests.

2.3 Sample Dates and Times: Test samples were collected on october 20, 22, and 25, 1993. Collection times were between 0940 and 1200 EDT. 
2.4 Sampling Hethod: All samples were collected using an Isco. peristaltic pump with the inlet tubing lowered to approximately 1.0-1.5 meters below the surface. Pumping rate was approximately four liters per minute. A 20 L composite was collected at each station and partitioned (split) according to the workplan for shipping to the TVA Toxicity Testing Laboratory (TTL) at Browns Ferry Nuclear Plant site or direct transfer to ORNL (DOE) personnel in the field.

2.5 Pertinent Site Conditions: Weather conditions were clear and sunny during sampling on October 20,22 , and 25 .

2.6 Sample Storage/Handling: All samples were placed on ice in ice chests after partitioning. Zero headspace was established in each container by filling full and expressing any air bubbles before sealing. Samples remained on ice until shipped or transferred directly to ORNL (DOE) personnel. Excess composite sample was discarded. Samples were stored in refrigerators at $4^{\circ} \mathrm{C} \pm 1^{\circ} \mathrm{C}$ after arrival at the TVA Toxicity Testing Laboratory (TTL).

2.7 Sample Transport: Samples collected on October 20,22 , and 25 were transported to the Browns Ferry Nuclear Plant site by overnight TVA mail courier. Personnel from ITL picked up samples from the mailroom and transported them to TTL. All chain of custody security seals were intact upon receipt of shipments.

2.8 Sample Pretreatment: Sample temperature was raised to $25^{\circ} \mathrm{C}$ in a warm water bath and samples were aerated as necessary to bring Do levels down to near 100 percent saturation. Adequate water for use in test initiation or daily renewal was filtered through a $64 \mu \mathrm{m}$ nylon mesh filter into $2000 \mathrm{~mL}$ beakers, and appropriate dilutions were prepared where applicable.

2.9 Test Treatments: Samples from Poplar Creek Hile 2.9, Mile 4.3, and Hile 22.0 were tested at 100 percent (undiluted) and diluted to 50 percent using moderately hard reconstituted water.

\subsection{TEST ORGANISMS/CULTURING CONDITIONS}

3.1 Species: Pimephales promelas, Fathead minnow

3.1.1 Source: TTL inhouse culture

3.1.2 Culture Water: Culture medium consisted of 50 percent dechlorinated tap water and 50 percent moderately hard reconstituted water. Dechlorination was achieved by activated carbon filtration and verified by DPD titration. Reagents for reconstituted water were added to reverse osmosis product water.

Both waters were passed through a pack column degasser to bring dissolved gases to saturation. Culture medium was continuously aerated to help ensure aseptic conditions. Total hardness was approximately $95 \mathrm{mg} / \mathrm{L}$ as $\mathrm{CaCO}_{3}$. 
$3.1 .3 \quad$ Temperature of Culture: $25^{\circ} \mathrm{C} \pm 1^{\circ} \mathrm{C}$

3.1.4 General Maintenance: Adult fathead minnows are maintained in glass aquaria in a flow through recirculating system. Flow rate to aquaria used for spawning is one aquarium volume per hour.

Approximately 20 percent of the water volume is replaced twice weekly. Adults are fed three times daily. Sexually mature fish are placed in 21-L glass aquaria (one male, four females) and reproduction is checked and recorded daily. Spawns are removed from aquaria and incubated in $1-L$ glass beakers under aeration to the proper stage of development for the target test.

Fish health is monitored regularly and corrective action is taken if necessary. Spawning frequency from individual aquaria is tracked and sexually spent individuals are replaced as necessary. Every 4-6 months a group of the same age fish from at least three spawns is reared to adults for replacement spawners.

3.1.5 Spawn Date: October 16, 1993

3.1.6 Hatch Date/Time: October 20, 1993/1000 CDT to October 21, 1993/0825 CDT

3.1.7 Culture/Acclimation Water: Moderately hard reconstituted water

3.1.8 Acclimation Temperature: $25^{\circ} \mathrm{C} \pm 1^{\circ} \mathrm{C}$

3.1 .9 Mean Dry Weight at Start of Test: $0.08 \mathrm{mg}$

3.1.10 Diseases and Treatment: None.

3.1.11 Food and Feeding: Larvae were fed brine shrimp (Artemia) nauplii $<24-h$ old twice daily beginning after hatching to ensure food availability if larvae began feeding prior to test initiation.

\subsection{TEST METHODS}

4.1 Fathead Minnow (Pimephales promelas) Larval Survival and Growth Test, EPA Test Method 1000.0. [3]

4.1.1 Modifications to/Deviations from Method 1000.0:

4.1.1.1 The Poplar Creek Mile 4.3 sample storage temperature on Day 6 was $2.7^{\circ} \mathrm{C}$ which was below the prescribed range of $4^{\circ} \mathrm{C} \pm 1^{\circ} \mathrm{C}$.

Temperatures of samples received on ice ranged from $0.4^{\circ} \mathrm{C}$ to $1.5^{\circ} \mathrm{C}$.

4.1.1.2 Temperatures in 10 beakers on Day 1 and one beaker on Day 7 were below the required $25^{\circ} \mathrm{C} \pm 1^{\circ} \mathrm{C}$. These deviate temperatures ranged from $23.5^{\circ} \mathrm{C}$ to $23.9^{\circ} \mathrm{C}$. No adverse affect to test results was likely since the low temperatures were random among treatments, and the overall means for the test $\left(24.9^{\circ} \mathrm{C}\right)$ and all individual treatments were within the EPA required limits.

$$
-3-
$$


4.1.2 Date/Time Test Initiated: October 21, 1993/0930 CDT

4.1.3 Date/Time Test Terminated: October 28, 1993/0945 CDT

4.1.4 Test Chamber: 600-mL borosilicate glass beakers

Volume per chamber: $350 \mathrm{~mL}$

4.1.5 Number of Test Organisms per Chamber: 10

4.1.6 Number of Replicates per Treatment: 4

4.1.7 Dilution Water/Control Water: Moderately hard reconstituted water

4.1.8 Renewal Period: 24-h

4.1.9 Test Temperature: $25.0^{\circ} \mathrm{C} \pm 1^{\circ} \mathrm{C}$

4.1.10 Feeding Regime During Test: Fathead minnow larvae were fed brine shrimp (Artemia) nauplii $<24-\mathrm{h}$ old 2 or 3 times daily ad libitum.

4.1.11 Physical and Chemical Parameters Measured: Parameters measured daily (initially) on test solutions and control waters were temperature (temperature was adjusted to equal "final" temperature before renewal), $\mathrm{DO}, \mathrm{pH}$ and conductivity. In addition, alkalinity, hardness, and total residual chorine were measured on each new sample at the time of first use $(10 / 21,10 / 23$, and $10 / 26)$.

Final measurements taken daily before renewal were temperature and $\mathrm{DO}$ in every test beaker and $\mathrm{pH}$ and conductivity in one replicate per treatment. Mean values and ranges are reported in section 6.3 .

\subsubsection{Statistics:}

Revised statistical procedures contained in the second edition of EPA's short-term chronic toxicity methods require a decision process for testing statistical assumptions before selecting a specific test method to determine toxicity endpoints. [3] Decision processes followed for testing survival and sublethal (growth) effects are shown in Sections 6.1.2.2 and 6.1.3.1. Based on tests for normal distribution and homogenous variance of data, the statistical test.used for endpoint determination of survival data was Steel's Many-one Rank Test and for growth data was Dunnett's Test.

\subsection{QUALITY ASSURANCE}

5.1 Toxicity Test Methods: All phases of the study including, but not limited to, sample collection, handling and storage; glassware preparation; test organism culturing/acquisition and acclimation; test organism handling during test; and maintaining appropriate test conditions were conducted according to the protocol as described in this report and EPA/600/4-89/001. [3] Any known deviations were noted during the study and are reported herein. 
5.2 Physical and Chemical Methods

5.2.1 Reagents, Titrants, Buffers, etc.: All chemicals were certified products used before expiration dates (where applicable). All TTL chemicals are recorded in a bound Laboratory Chemical Logbook and specific chemicals used were documented on a chemical record sheet contained in the study notebook.

5.2.2 Instruments: All identification, service, calibration, and standardization information pertaining to TTL laboratory instruments is contained in bound Laboratory Instrument Logbooks and specific instruments used were documented on an instrument record sheet contained in the study notebook.

5.2.3 Temperature was measured using glass mercury thermometers. The instrument was standardized and inspected with readings made according to TVA procedures ES-43.8 and ES-42.11. [4]

5.2.4 Dissolved oxygen was measured using a YSI Model 57 oxygen meter. The instrument was standardized (using the Winkler method) and readings were taken according to TVA procedures ES-43.6 and ES-42.4, respectively. [4]

5.2.5 The $\mathrm{pH}$ was measured using an Orion Hodel SA250 meter equipped with an Orion Ross combination electrode. The instrument was standardized and readings were made according to TVA procedures ES-43.7 and DS-42.8, respectively. [4]

5.2.6 Conductance was measured using a YSI Hodel 32 SCT meter. The instrument was standardized and readings were taken according to TVA procedures ES-43.3 and ES-42.3, respectively. [4]

5.2.7 Alkalinity was measured by titration of $100 \mathrm{~mL}$ samples with 0.02 $\mathrm{H}_{2} \mathrm{SO}_{4}$ to an end point of 4.5 according to TVA procedure ES-42.1. [4]

5.2.8 Hardness was determined by titration of $50 \mathrm{~mL}$ samples with EDTA to a colorimetric endpoint using an indicator (Instructions provided by Reagent Manufacturer [Calgon]), Schwarzenbach Method.

5.2.9 Total residual chlorine (TRC) was determined using the DPD Titrimetric Method according to TVA procedure ES-42.9, Rev. 0. [4]

5.3 Reference Toxicant Tests

5.3.1 Test Type: Fish - 7-day chronic (MOEC)

5.3.2 Standard Toxicants Used:

Copper Sulfate Reference Toxicant Solution Source/Brand: EPA

5.3.3 Dilution Water Used: Moderately hard reconstituted water 
5.3.4 Statistics: Chronic Test, FH Survival/Growth, woEC -

Dunnett's Test or Steel's Many-one Rank Test, EPA Bootstrap Procedure - $\mathrm{IC}_{25}$

$6.0 \quad$ RESULTS

6.1 Fathead Minnow Larval Survival and Growth Test

6.1.1 Sumary of Results: No survival or growth effects were demonstrated in fathead minnows exposed to Poplar Creek Mile 2.9, Mile 4.3, or Mile 5.1 samples during the CR-ERP Ambient water Toxicity study conducted from October 21-28, 1993.

6.1.2 Results, Survival Data:

6.1.2.1 Daily Percent Survival Summary for Fathead Minnow Larval Survival Test, CR-ERP Study, October 21-28, 1993.

\begin{tabular}{lrrrrrrrr}
\hline Treatment & \multicolumn{7}{c}{ Total Daily \% Survival } \\
\cline { 2 - 8 } & 1 & 2 & 3 & 4 & 5 & 6 & 7 \\
\hline Medium & 100 & 100 & 100 & 100 & 100 & 100 & 100 \\
PCH 2.9-50\% & 100 & 100 & 100 & 98 & 98 & 98 & 98 \\
PCH 2.9-100\% & 100 & 100 & 100 & 98 & 98 & 98 & 98 \\
PCH 4.3-50\% & 100 & 100 & 100 & 98 & 97 & 97 & 97 \\
PCH 4.3-100\% & 100 & 100 & 100 & 98 & 98 & 98 & 98 \\
PCH 5.1-50\% & 100 & 100 & 100 & 93 & 93 & 93 & 93 \\
PCH 5.1-100\% & 100 & 100 & 100 & 100 & 100 & 100 & 100
\end{tabular}

* Replicate 4 counted as 9 organisms initially for Days 5,6 , and 7 due to accidental injury/death of $1 \mathrm{fish}$.

6.1.2.2 Statistical Decision Process for Determining Toxicity Endpoints for 7-Day Survival of Fathead Minnows, CR-ERP Study, October 21-28, . 1993.

SURVIVAL EFFECTS

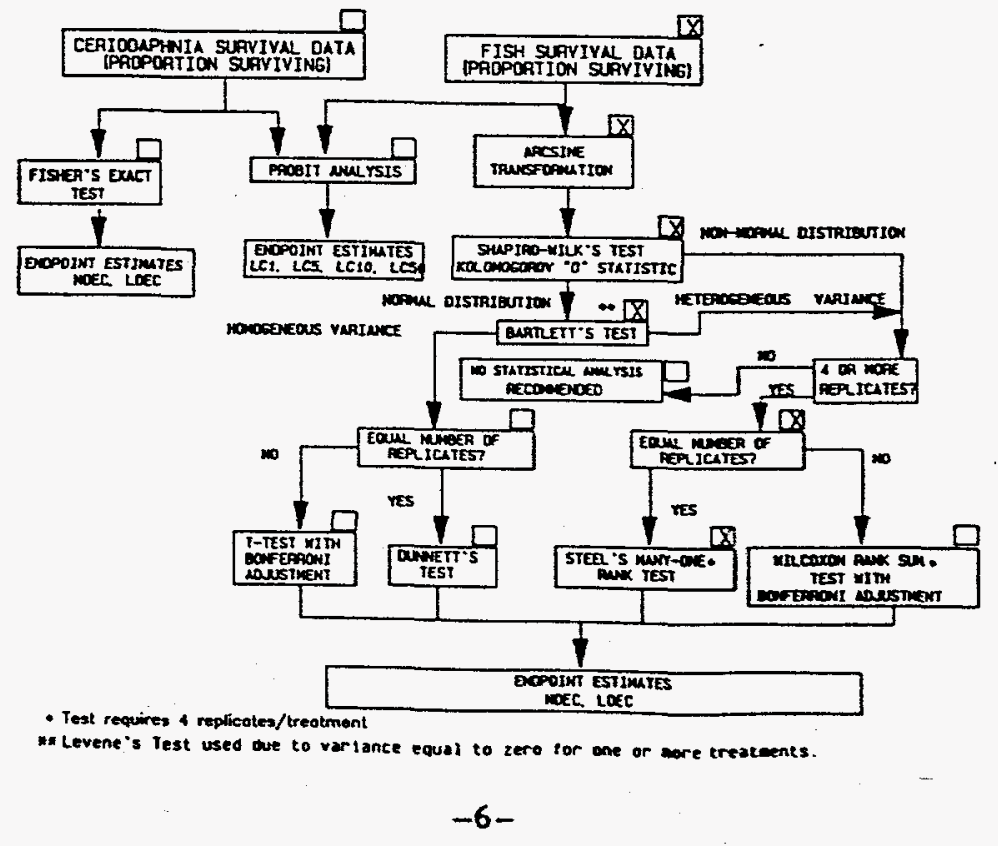


6.1.2.3 Analysis of Survival Data Using Steel's Hany-one Rank Test for Fathead Minnow 7-Day Larval Survival Test, CR-ERP Study, October 21-28, 1993.

\begin{tabular}{|c|c|c|c|c|c|c|c|c|c|c|c|c|}
\hline & & & & & & & & & & & & \\
\hline & & & & Surv & al & \%) & ata & & & & & \\
\hline & & & & & $\mathrm{pli}$ & ate & & & & & & \\
\hline Group & 1 & 2 & 3 & 4 & 5 & 6 & 7 & 8 & 9 & 10 & Hean & $s^{*}$ \\
\hline Hedium & 100 & 100 & 100 & 100 & & & & & & $\ldots$ & 100 & \\
\hline PCY 2.9-50\% & 100 & 100 & 100 & 90 & & & & & & & 98 & \\
\hline PCU 2.9-100\% & 90 & 100 & 100 & 100 & & & & & & & 98 & \\
\hline PCH 4.3-50\% & 100 & 100 & 100 & 89 & & & & & & & 97 & \\
\hline PCH 4.3-100\% & 100 & 100 & 90 & 100 & & & & & & & 98 & \\
\hline PGI 5.1-50\% & 100 & 100 & 100 & 70 & & & & & & & 93 & \\
\hline PCI 5.1-100\% & 100 & 100 & 100 & 100 & & & & & & & 100 & \\
\hline
\end{tabular}

Steel's Many-one Rank Test

\begin{tabular}{lccc}
\hline \multicolumn{5}{c}{ Treatment } & Replicates & Critical Rank Sum & Rank Sum \\
\hline & 4 & 10 & \\
PCH 2.9-50\% & 4 & 10 & 16.0 \\
PCY 2.9-100\% & 4 & 10 & 16.0 \\
PCH 4.3-50\% & 4 & 10 & 16.0 \\
PCH 4.3-100\% & 4 & 10 & 16.0 \\
PCI 5.1-50\% & 4 & 10 & 16.0 \\
PCI 5.1-100\% & 4 & & 18.0
\end{tabular}

*Values less than or equal to the Critical Rank Sum are significantly less than the control (Medium).

6.1.3 Results, Growth Data:

6.1.3.1 Statistical Decision Process for Determining Toxicity Endpoints for 7-Day Growth of Fathead Hinnows, CR-ERP Study, October 21-28, 1993.

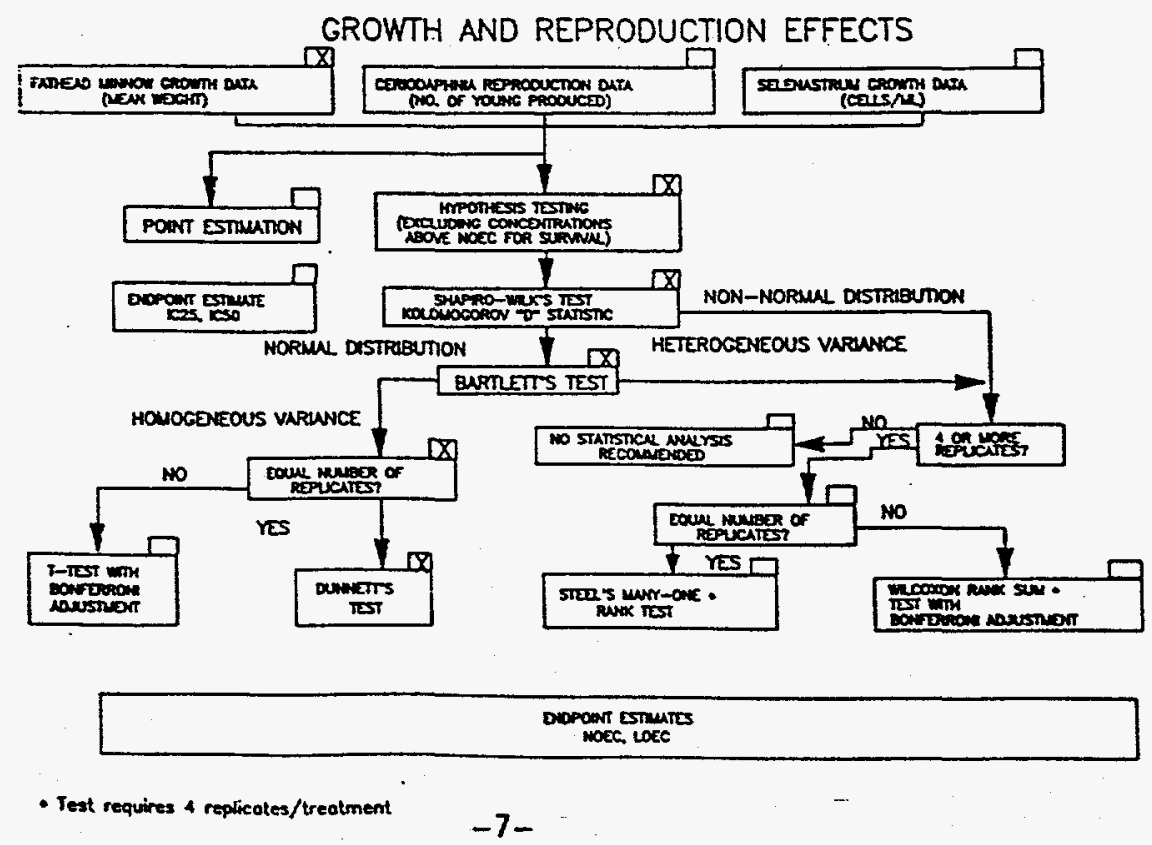


6.1.3.2 Analysis of Dry Weight (mg) Data Using Dunnett's Test for Fathead Minnow 7-Day Larval Growth Test, CR-ERP Study, October 21-28, 1993.

Wo transformation applied before data analysis. For this set of data, the minimm significant difference is 0.08 .

This represents an $15.51 \%$ decrease in dry weight, mg.

$$
T=2.45 \quad \text { Alpha }=0.05 \quad \text { (one-tailed test) }
$$

\begin{tabular}{|c|c|c|c|c|c|c|c|c|c|c|c|c|}
\hline \multirow{3}{*}{ Treatment } & \multicolumn{10}{|c|}{ Growth Data (mg) } & \multirow{3}{*}{ Mean } & \multirow{3}{*}{$s \star$} \\
\hline & \multicolumn{10}{|c|}{ Replicate } & & \\
\hline & 1 & 2 & 3 & 4 & 5 & 6 & 7 & 8 & 9 & 10 & & \\
\hline $\begin{array}{l}\text { Hedium } \\
\text { PCK 2.9-50\% }\end{array}$ & $\begin{array}{l}0.51 \\
0.51\end{array}$ & $\begin{array}{l}0.47 \\
0.49\end{array}$ & $\begin{array}{l}0.54 \\
0.53\end{array}$ & $\begin{array}{l}.0 .46 \\
0.50\end{array}$ & & & & & & & $\begin{array}{l}0.50 \\
0.51\end{array}$ & \\
\hline $\begin{array}{l}\text { PCE } 2.9-100 \% \\
\text { PCE } 4.3-50 \%\end{array}$ & $\begin{array}{l}0.50 \\
0.58\end{array}$ & $\begin{array}{l}0.48 \\
0.58\end{array}$ & $\begin{array}{l}0.50 \\
0.53\end{array}$ & $\begin{array}{l}0.62 \\
0.61\end{array}$ & & & & & & & $\begin{array}{l}0.53 \\
0.57\end{array}$ & \\
\hline $\begin{array}{l}\text { PCH 4.3-100\% } \\
\text { PCH 5.1-50\% }\end{array}$ & $\begin{array}{l}0.54 \\
0.46\end{array}$ & $\begin{array}{l}0.49 \\
0.50\end{array}$ & $\begin{array}{l}0.49 \\
0.44\end{array}$ & $\begin{array}{l}0.39 \\
0.54\end{array}$ & & & & & & & $\begin{array}{l}0.48 \\
0.48\end{array}$ & \\
\hline PCL 5.1-100\% & 0.53 & 0.46 & 0.52 & 0.50 & & & & & & & 0.50 & \\
\hline
\end{tabular}

*Values significantly less than the control (Medium). EMS $=0.002$.

6.2 Physical/Chemical Parameters

6.2.1 Overall Test Temperature: Fathead Minnow $-24.9^{\circ} \mathrm{C}\left(23.5^{\circ}-26.0^{\circ} \mathrm{C}\right)$

6.2.2 Water chemistry summary for CR-ERP Study, October 21-28, 1993.

See: Appendix A Water Chemistry Hean Values and Ranges for Fathead Minnow Test, CR-ERP Study, October 21-28, 1993

6.3 Reference Toxicant Tests

6.3.1 Summary of Results:

Reference toxicant tests conducted prior to CR-ERP study showed chronic results consistent with TTL control chart ranges for fathead minnows.

6.3.2 Fathead Minnows

6.3.2.1 Date/Time of Most Recent Test:

September 30, 1993/0915 CDT to October 7, 1993/0830 CDT

6.3.2.2 LOEC: $0.008 \mathrm{mg} \mathrm{Cu/L}$

NOBC: $0.003 \mathrm{mg} \mathrm{Cu} / \mathrm{L}$

$\mathrm{IC}_{25}: 0.006 \mathrm{mg} \mathrm{Cu} / \mathrm{L}$ 
6.3.2.3 Control Chart Information:

Number of standard tests completed by laboratory: 48

LOEC Range: $0.008-0.02 \mathrm{mg} \mathrm{Cu} / \mathrm{L}$ (one concentration increment)

WOEC Range: $0.003-0.008 \mathrm{mg} \mathrm{Cu} / \mathrm{L}$ (one concentration increment)

$\mathrm{IC}_{25}$ Range: $0.004-0.012 \mathrm{mg} \mathrm{Cu} / \mathrm{L}$

$\underline{7.0 \quad \text { CONCLUSION }}$

Testing conducted using Poplar Creek Mile 2.9, Mile 4.3, and Mile 5.1 samples collected on october 20,22 , and 25 showed no toxicity (survival or growth) to fathead minnows.

\subsection{REFERENCES}

1. Phipps, T. L., and L. A. Kszos, Statement of work, Environmental Sciences Division, Biomonitoring Group, Oak Ridge Wational Laboratory (April 1993, revised June 1993).

2. Letter, J. Moses, TVA, to Terry L. Phipps, ORNL, October 14, 1993, Re: Using Only Fathead Minnows for October 1993; Rescheduling Daphnid Test for 1994.

3. Weber, C. I., W. H. Peltier, T. J. Norberg-King, W. B. Horning, F. A. Kessler, J. R. Menkdick, T. W. Neiheisel, P. A. Lewis, D. J. Klem, Q. H. Pickering, F. L. Robinson, J. H. Lazorchak, L.

J. Wymer, and R. W. Freyberg.. Short-Term Methods for Estimating the Chronic Toxicity of Effluents and Receiving Waters to Freshwater Organisms. EPA/600/4-89/001 (March 1989) and EPA/600/4-89/001a (September 1989).

4. Field Operations Natural Resource Engineering Procedures Manual, Vol. 1, Division of Natural Resource Operations, Tennessee Valley Authority.

PLARC501-1951 
Appendix A

Water Chemistry Mean Values and Ranges for Fathead Minnow Test, CR-ERP Ambient Water Toxicity Study, October 21-28, 1993

\begin{tabular}{|c|c|c|c|c|c|c|c|c|c|c|}
\hline \multirow[b]{2}{*}{ Source } & \multirow{2}{*}{$\begin{array}{c}\frac{\text { Iemperature }}{\text { Final }} \\
\text { Fish }\end{array}$} & \multicolumn{2}{|c|}{ Dissolved Oxygen } & \multicolumn{2}{|c|}{$\mathrm{pH}$} & \multicolumn{2}{|c|}{ Conductivity } & \multirow{2}{*}{ 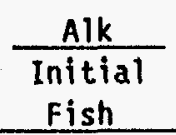 } & \multirow{2}{*}{$\begin{array}{c}\text { Hardness } \\
\text { Initial } \\
\text { Fish }\end{array}$} & \multirow{2}{*}{$\frac{\text { Chlorine }}{\text { Initial }}$} \\
\hline & & $\begin{array}{l}\text { Initial } \\
\text { Fish } \\
\end{array}$ & $\begin{array}{l}\text { Final } \\
\text { Fish }\end{array}$ & $\begin{array}{l}\text { Initial } \\
\text { Fish } \\
\end{array}$ & $\begin{array}{l}\text { Final } \\
\text { Fish } \\
\end{array}$ & $\begin{array}{l}\text { Initial } \\
\text { Fish }\end{array}$ & $\begin{array}{l}\text { Final } \\
\text { Fish }\end{array}$ & & & \\
\hline & $\left({ }^{\circ} \mathrm{C}\right)$ & $(\mathrm{mg} / \mathrm{L})$ & $(\mathrm{mg} / \mathrm{L})$ & (S.U.) & (5.U.) & (umhos) & (umhos) & $\star$ & $\star$ & $(m g / L)$ \\
\hline Fish Medium & $\begin{array}{c}24.9 \\
(23.5-25.9)\end{array}$ & $\begin{array}{c}8.3 \\
(8.1-8.4)\end{array}$ & $\begin{array}{c}6.0 \\
(4.6-7.1)\end{array}$ & $\begin{array}{c}8.2 \\
(8.2-8.3)\end{array}$ & $\begin{array}{c}7.8 \\
(7.6-7.9)\end{array}$ & $\begin{array}{c}348 \\
(338-360)\end{array}$ & $\begin{array}{c}352 \\
(344-363)\end{array}$ & $\begin{array}{c}67 \\
(65-68)\end{array}$ & $\begin{array}{c}94.1 \\
(94.1-94.1)\end{array}$ & - \\
\hline PCM 2.9-50\% & $\begin{array}{c}24.9 \\
(23.9-26.0)\end{array}$ & $\begin{array}{c}8.3 \\
(8.2-8.4)\end{array}$ & $\begin{array}{c}6.0 \\
(4.5-7.0)\end{array}$ & $\begin{array}{c}7.9 \\
(7.1-8.0)\end{array}$ & $\begin{array}{c}7.9 \\
(7.7-8.0)\end{array}$ & $\begin{array}{c}360 \\
(353-366)\end{array}$ & $\begin{array}{c}364 \\
(356-371)\end{array}$ & - & - & - \\
\hline PCM 2.9-100\% & $\begin{array}{c}24.9 \\
(23.9-25.9)\end{array}$ & $\begin{array}{c}8.3 \\
(8.0-8.4)\end{array}$ & $\begin{array}{c}6.0 \\
(4.8-7.1)\end{array}$ & $\begin{array}{c}7.7 \\
(7.5-8.0)\end{array}$ & $\begin{array}{c}8.0 \\
(7.8-8.1)\end{array}$ & $\begin{array}{c}375 \\
(363-382)\end{array}$ & $\begin{array}{c}378 \\
(370-384)\end{array}$ & $\begin{array}{c}116 \\
(113-119)\end{array}$ & $\begin{array}{c}153.9 \\
(153.9-153.9)\end{array}$ & $\begin{array}{c}<0.1 \\
(<0.1-<0.1)\end{array}$ \\
\hline PCM 4.3-50\% & $\begin{array}{c}24.8 \\
(23.8-25.8)\end{array}$ & $\begin{array}{c}8.2 \\
(8.0-8.4)\end{array}$ & $\begin{array}{c}6.0 \\
(4.3-7.0)\end{array}$ & $\begin{array}{c}7.7 \\
(7.6-7.8)\end{array}$ & $\begin{array}{c}7.9 \\
(7.7-8.0)\end{array}$ & $\begin{array}{c}375 \\
(368-382)\end{array}$ & $\begin{array}{c}378 \\
(371-389)\end{array}$ & - & - & - \\
\hline PCM 4.3-100\% & $\begin{array}{c}24.9 \\
(23.7-25.9)\end{array}$ & $\begin{array}{c}7.6 \\
(7.0-8.1)\end{array}$ & $\begin{array}{c}5.9 \\
(4.5-7.1)\end{array}$ & $\begin{array}{c}7.5 \\
(7.3-7.6)\end{array}$ & $\begin{array}{c}7.9 \\
(7.8-8.0)\end{array}$ & $\begin{array}{c}406 \\
(401-416)\end{array}$ & $\begin{array}{c}410 \\
(405-421)\end{array}$ & $\begin{array}{c}122 \\
(120-126)\end{array}$ & $\begin{array}{c}160.2 \\
(159.0-162.5)\end{array}$ & $\begin{array}{c}<0.1 \\
(<0.1-<0.1)\end{array}$ \\
\hline PCH 5.1-50\% & $\begin{array}{c}24.8 \\
(23.7-25.8)\end{array}$ & $\begin{array}{c}8.2 \\
(8.0-8.4)\end{array}$ & $\begin{array}{c}6.1 \\
(4.1-7.2)\end{array}$ & $\begin{array}{c}7.7 \\
(7.6-7.8)\end{array}$ & $\begin{array}{c}7.9 \\
(7.7-8.0)\end{array}$ & $\begin{array}{c}371 \\
(367-375)\end{array}$ & $\begin{array}{c}378 \\
(371-385)\end{array}$ & - & - & - \\
\hline PCM 5.1-100\% & $\begin{array}{c}24.9 \\
(23.5-25.9)\end{array}$ & $\begin{array}{c}7.6 \\
(6.9-8.3)\end{array}$ & $\begin{array}{c}6.0 \\
(4.7-7.2)\end{array}$ & $\begin{array}{c}7.5 \\
(7.3-7.6)\end{array}$ & $\begin{array}{c}7.9 \\
(7.8-8.0)\end{array}$ & $\begin{array}{c}401 \\
(397-405)\end{array}$ & $\begin{array}{c}405 \\
(401-410)\end{array}$ & $\begin{array}{c}122 \\
(120-127)\end{array}$ & $\begin{array}{c}161.3 \\
(159.0-162.5)\end{array}$ & $\begin{array}{c}<0.1 \\
(<0.1-<0.1)\end{array}$ \\
\hline
\end{tabular}


ATTACHMENT I

CR-ERP AMBIENT WATER TOXICITY STUDY Chain-of-Custody Forms - Originals 
FIELD CHAIN OF CUSTODY TUA $\rightarrow$ TVAT

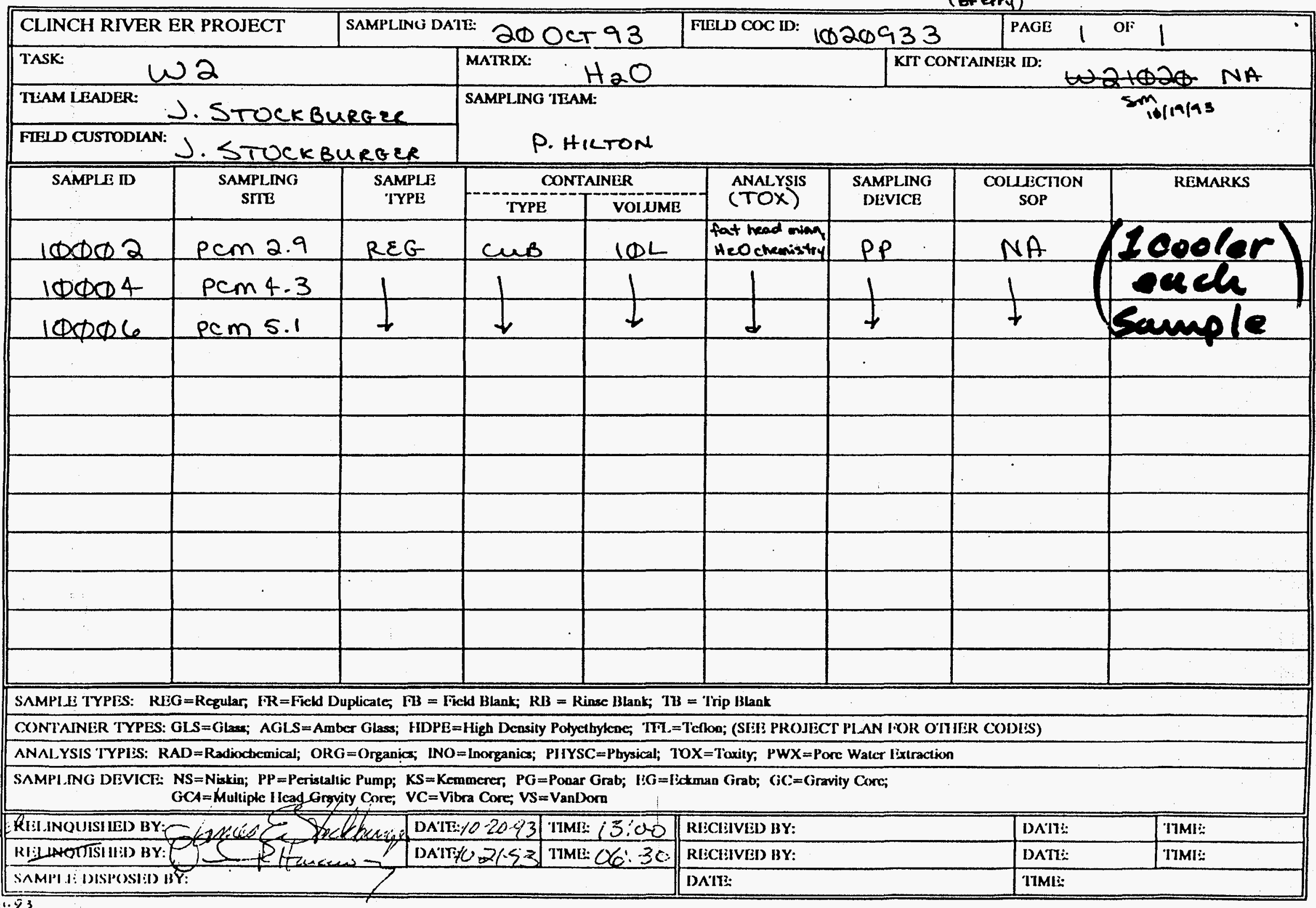




\section{FIELD CHAIN OF CUSTODY IVA $\rightarrow$ BFerry}

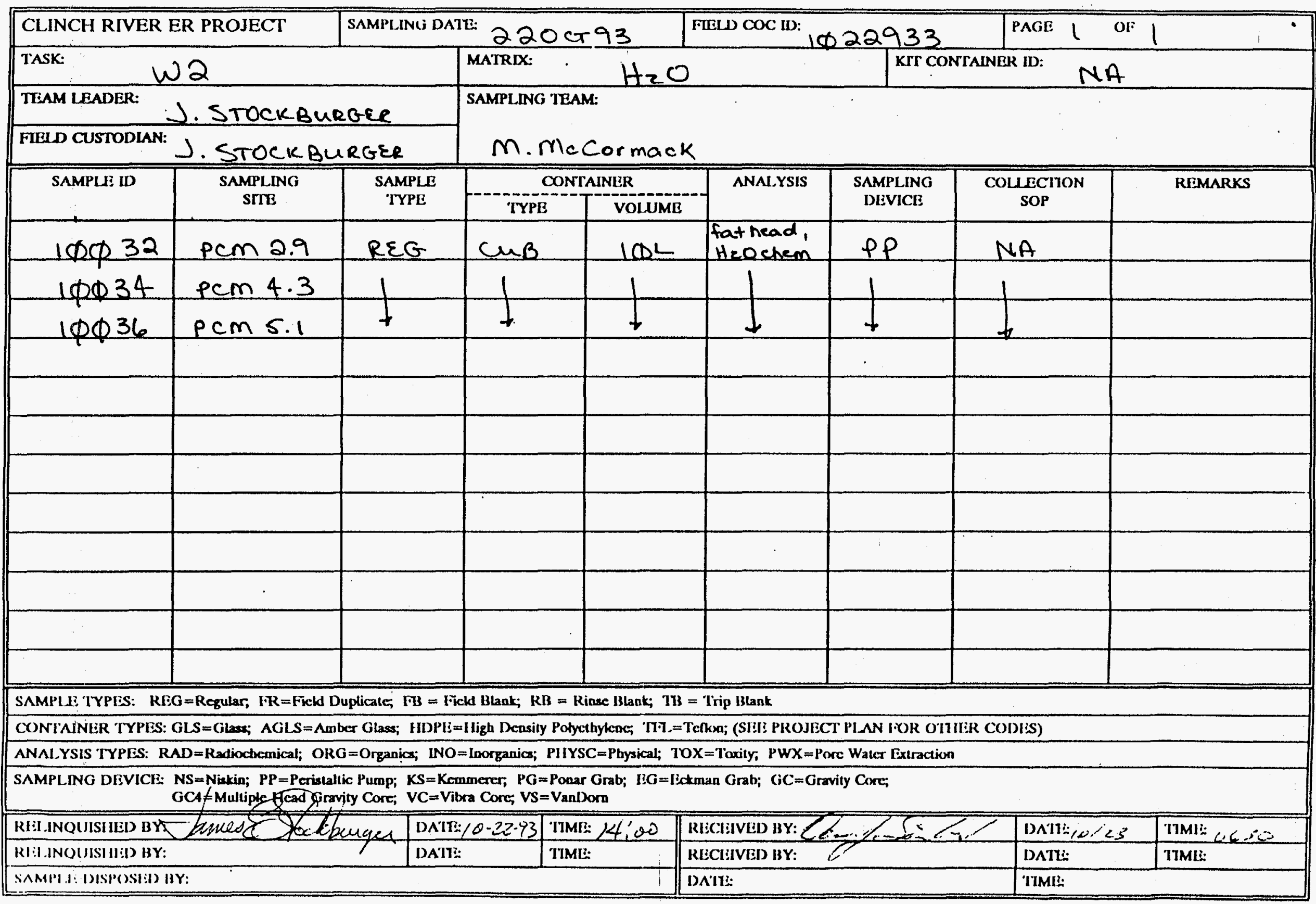


FIELD CHAIN OF CUSTODY TVA $\rightarrow$ B ferry

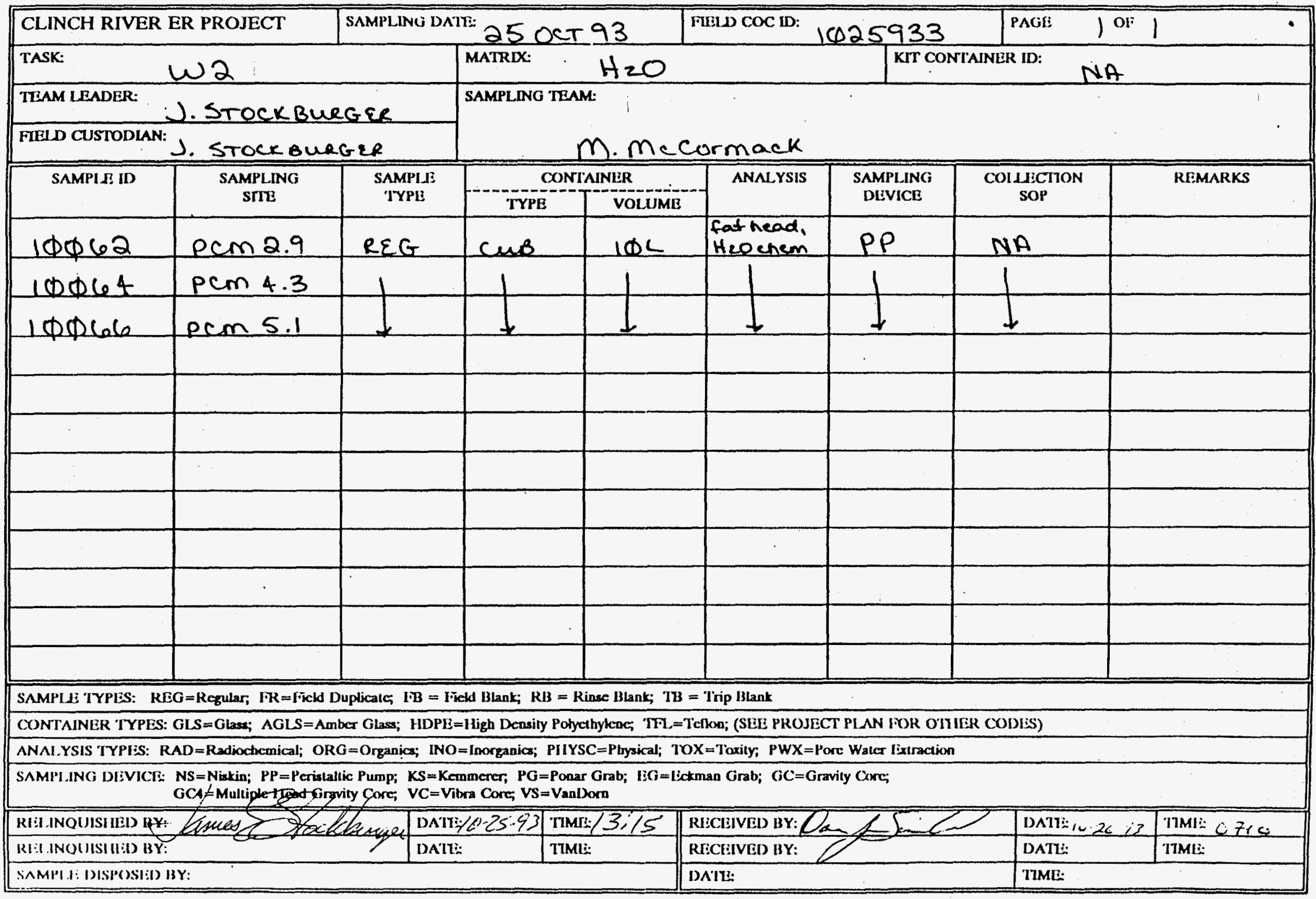

Ifc: ce: Rev. 2 21Slipy

DOCUMIINI ID: 
ATTACHMENT II

CR-ERP AMBIENT WATER TOXICITY STUDY

Toxicity Test Bench Sheets and Statistical Analyses 
ARL CHRONIC TOXICITY TEST MASTER ChECKLIST study: DOE Ambient ToX Date Issued: $10-20$ To: DJS From: Tm

1. Test organism availability: FH Minnows-T.L.* JM/ c.u.+TCS Date 9-29 Daphnids - T.L. N/A T.U. Date $\rightarrow$

2. Sample collection Coordination: Date Accondi to Sow with

3. Glassware availability: T.L. g/5 Tech. Date $10-20$

4. Glassware prep. complete: Date $1 ;-20$ : By y/S

5. Data Sheet prep. complete: Date 10-20 By 015

6. Test organism availability verification: FH-OK? 825 Daphnids-OK? $N / A$

7. FH Minnow acclimation:

Day 12

By NA NA

8. Test Initiation: FH-Date/Time 12-21-83/0930 Daphnid-Date/Time Initial Chem.

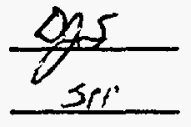
FH FH Daphnid

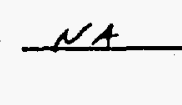

9. Test Renewal: Day \#: I Initial Chem. - T.L. OHS $\frac{\text { OgS }}{\text { TPP }}$ T.u. IPP IPP Log Samples

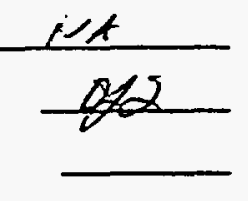
Final Chert.
T.L.
T.M. JPP
F.H.
- T.L. $\frac{\Delta / S}{\partial L}$
T.H. IPP

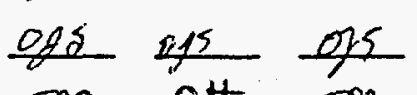
spe ot
or
JPD 12
curfak JPP

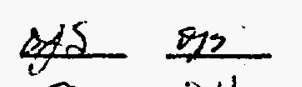
JPP
$\frac{\text { Jp }}{\text { un/ax JPP }}$
Jos
SPP

Daphnid

$$
\text { - T.L. } N A
$$

T.H. $N A$

456

(7)

(8)

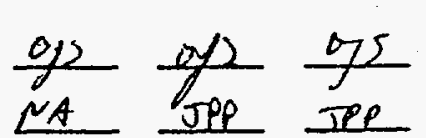

$\operatorname{IeP}_{\text {cuefoK NA }}^{N A}$

$\longrightarrow$
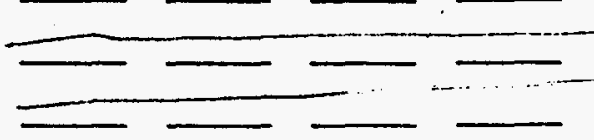

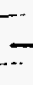

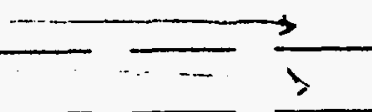

10. Test Termination: FH-Date/Time 10.78.93/n945 Daphnid-Date/Time

Final Chem.

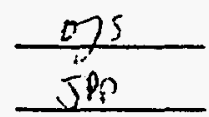

FH $\frac{0,55}{\frac{i}{i n}}$ Daphnid

$N A$ Clean-up 195

$N A$ Net

11. Weigh Fish: Date $\frac{10-29.93}{11.04 .93}:$ By $\frac{\text { oper }}{\mathrm{Con}}$

12. Run Stats: Date $11-03 \cdot i_{3}$ By $\mathrm{Cn}$

13. Report Prep.: Date $11-15-93$ By $\frac{0 \alpha 5}{0}$

14. Report Final: Date $11-24-93$ By Th Approved: $\mathbb{N} \mathbb{H}^{4}$

*Team Leader tculture Unit \$Team Member 
Chronic Study Fathead Record sheet

study CR-ERP QA Test October 1993

Test organism Pimephales promelas

Beginning Date/Time $10.21-93 / 0930$

Ending Date/Time $10.28-93 / 0945$

Personnel Simbeck, Posey, Kinard, hussel

Control/Dilution water MHRY (E-MED)

Test Treatment Identification

1. E-MEQ (control)

2. $\rho C M 2.250 \%$

3. $\operatorname{lem} 2.9100 \%$

4. $\operatorname{pem~} 4.350 \%$

5. PCM $4.3 \quad 100 \%$

6. $\operatorname{RCM~} 5.150 \%$

7. $\operatorname{sen} 5.1 \quad 100 \%$

8.

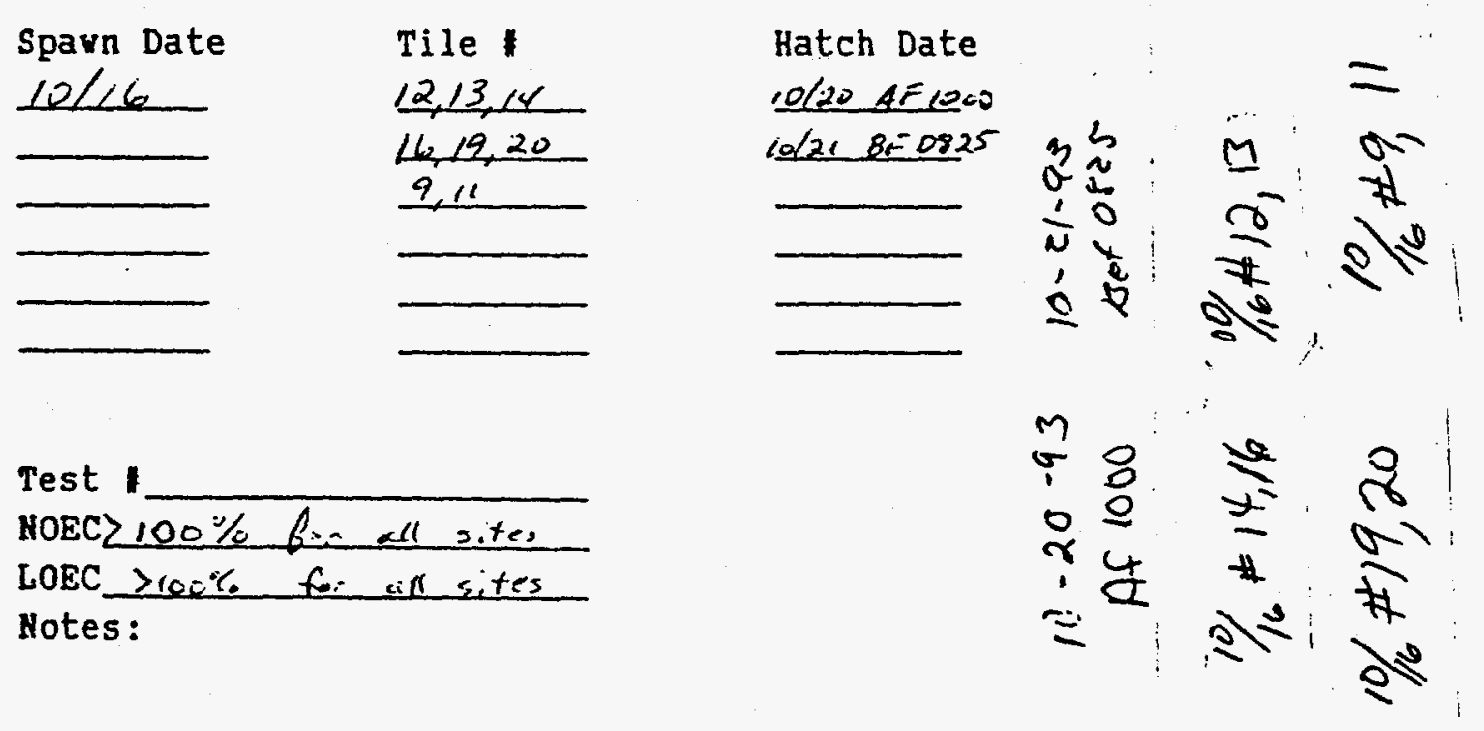




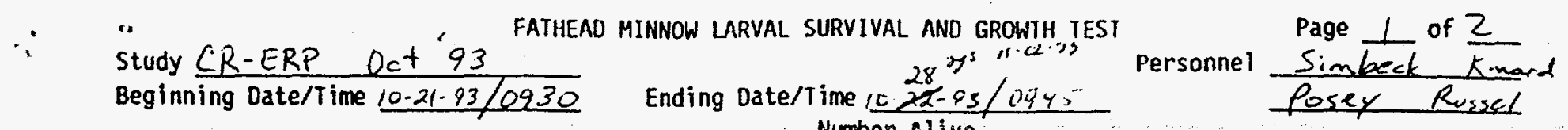

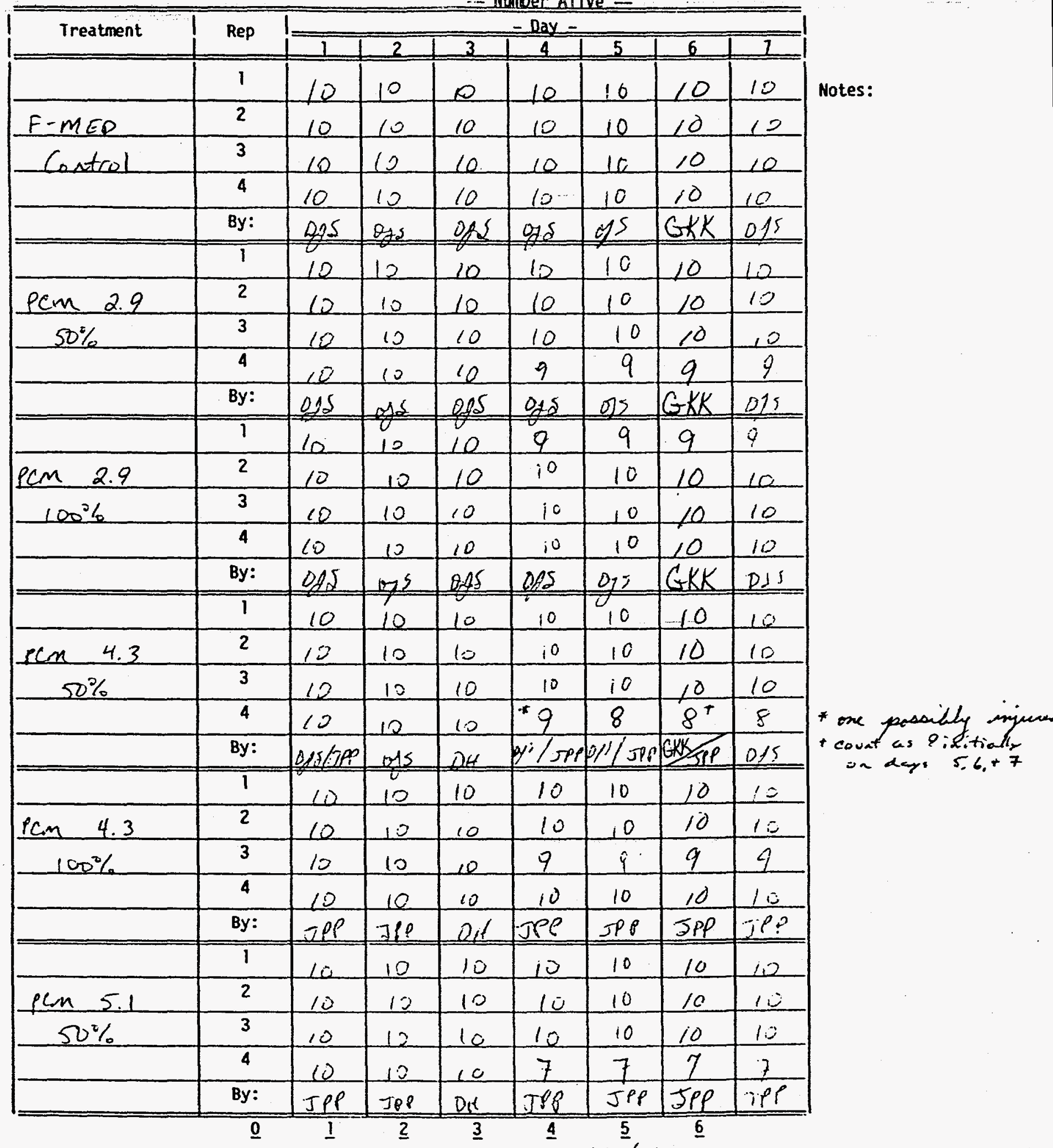

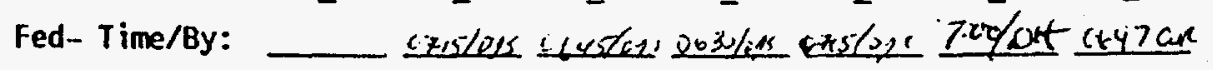

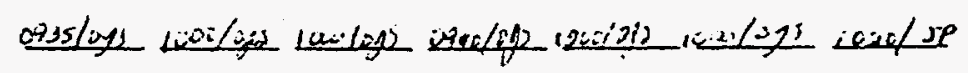

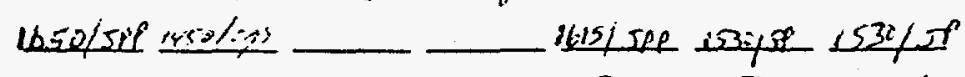

Dilution Water 10: $1503 \quad 1.20 ; 1503 \quad 1503 \quad 1504-15041504$

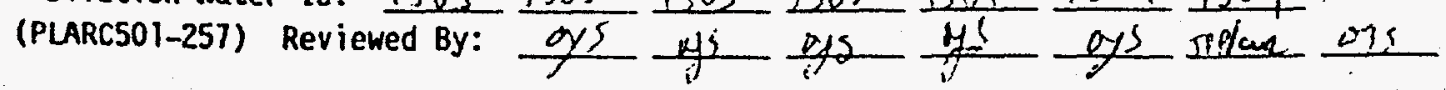


FATHEAD MINNOW LARVAL SURVIVAL AND GROWTH TEST

Study $C R$-ERO $\mathrm{CKT}^{\circ} \mathrm{Q}_{3}$ Beginning Date/Time 10.21-93/0932
Ending DaterTime $10.21 \cdot 93 / 0745$

Personnel - Number Alive -
Page 2 of 2 Simbeck Kabou Posey Rorsed

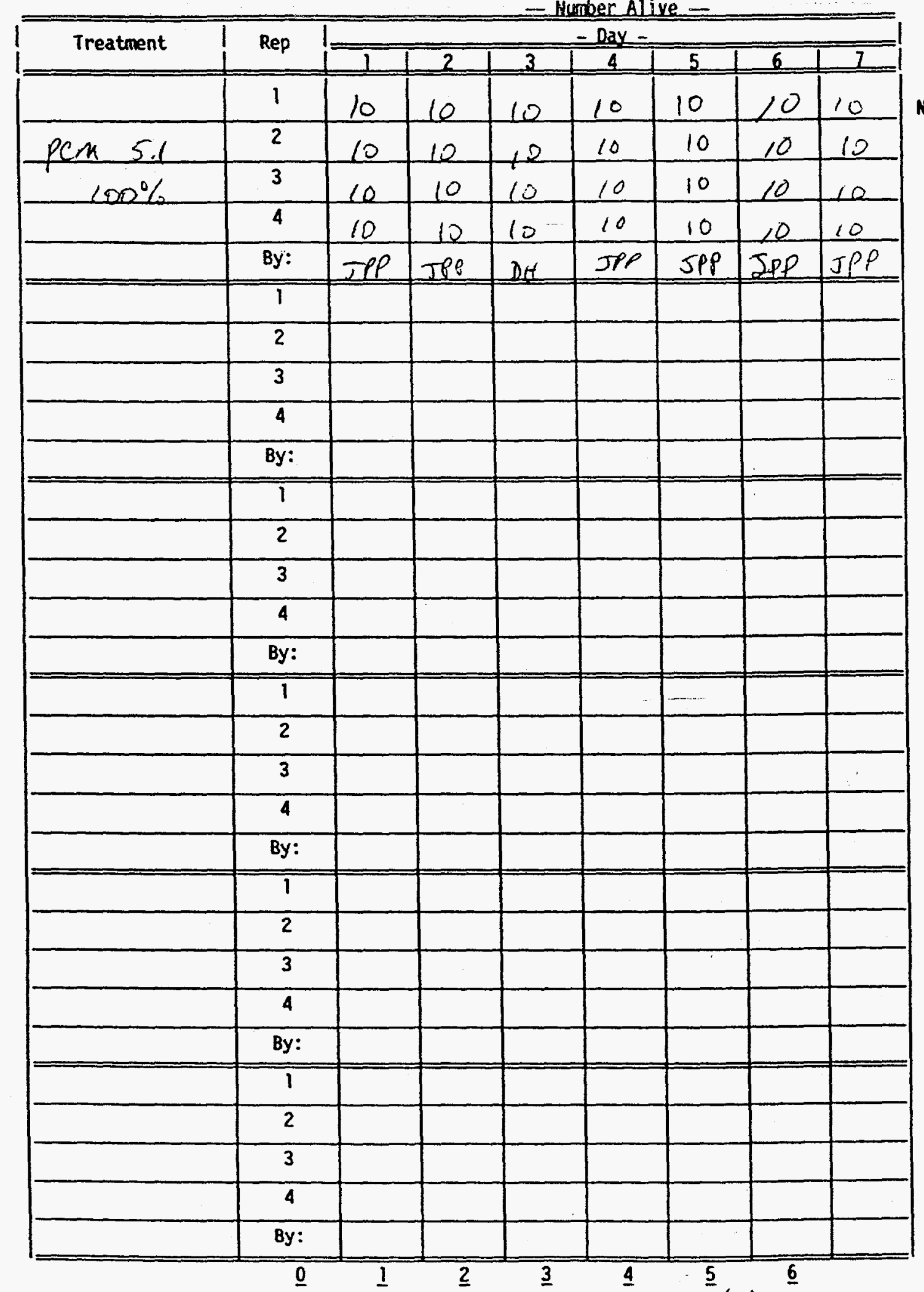

Notes:

Fed-Time/By:

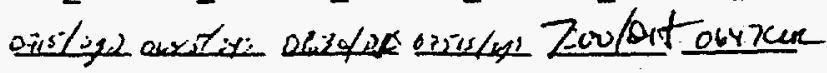

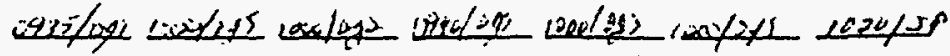

1650/2e 1450/30s 1615/5r 133015m 1530\%

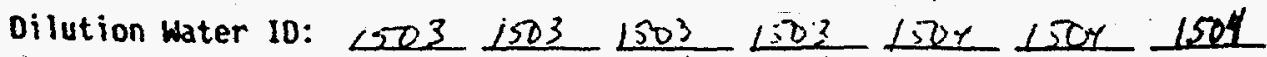

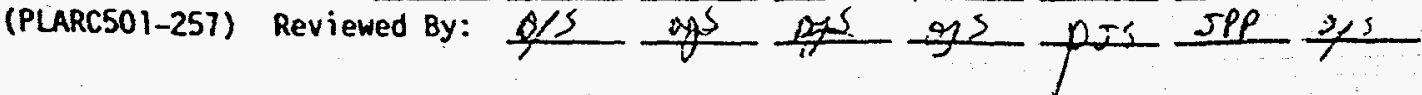


Analysis of Survival

CR-ERP \#3

Starting Date: 10/21/93

Survival (\%) Data

-................-

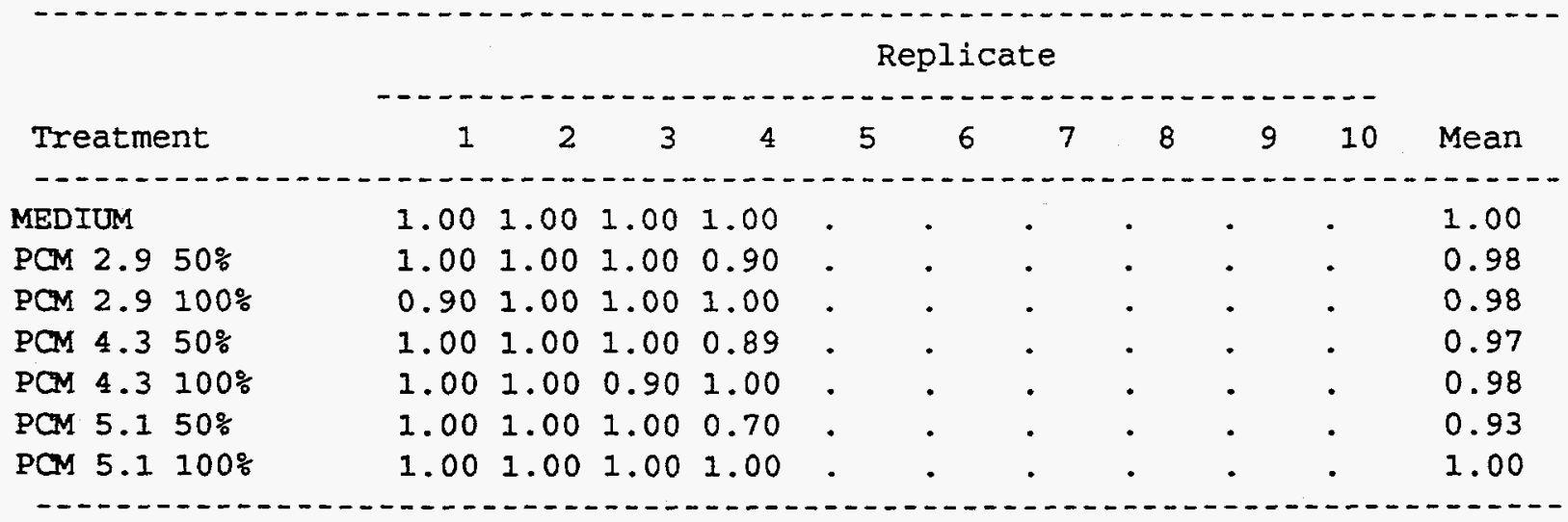

Steel's Many-one Rank Test

\begin{tabular}{|c|c|c|c|}
\hline Treatment & $\begin{array}{l}\text { No. of } \\
\text { Replicates }\end{array}$ & $\begin{array}{l}\text { Critical } \\
\text { Rank Sum }\end{array}$ & Rank Sum* \\
\hline PCM $2.950 \%$ & 4 & 10 & 16 \\
\hline PCM $2.9 \quad 100 \%$ & 4 & 10 & 16 \\
\hline PCM $4.350 \%$ & 4 & 10 & 16 \\
\hline PCM $4.3 \quad 100 \%$ & 4 & 10 & 16 \\
\hline PCM $5.150 \%$ & 4 & 10 & 16 \\
\hline PCM $5.1 \quad 100 \%$ & 4 & 10 & 18 \\
\hline
\end{tabular}

* Values less than Critical Rank Sum significantly less than the control (MEDIUM ). 
20:37 Sunday, October 27, 1991

STUDY=CR-ERP \#3 ST_DATE=102193 PARM=MINS TEST=STEEL $\ldots \ldots \ldots \ldots \ldots$

$\begin{array}{llccccc}\text { OBS } & \text { TREATMENT } & \text { N CONTROL } & \text { N EFFLUENT } & \text { RANK SUM } & \text { CRITIC RANK SU } \\ & & & & & & \\ 1 & \text { PCM 2.9 50\% } & 4 & 4 & 16 & 10 \\ 2 . & \text { PCM 2.9 100\% } & 4 & 4 & 16 & 10 \\ 3 & \text { PCM 4.3 50\% } & 4 & 4 & 16 & 10 \\ 4 & \text { PCM 4.3 100\% } & 4 & 4 & 16 & 10 \\ 5 & \text { PCM 5.1 50\% } & 4 & 4 & 16 & 10 \\ 6 & \text { PCM 5.1 100\% } & 4 & 4 & 18 & 10\end{array}$




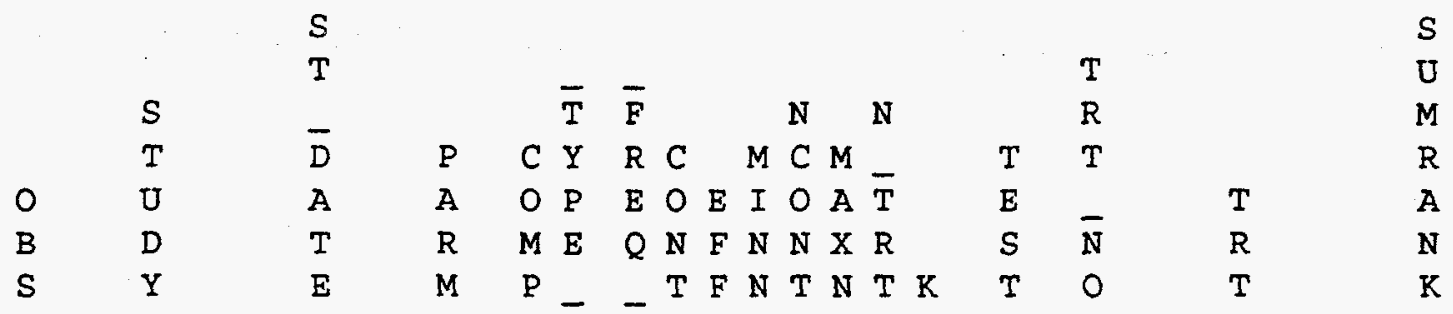

1 CR-ERP \#3 102193 MINS 20010444444466 STEEL 1 PCM $2.950 \% 20$ 2 CR-ERP \#3 102193 MINS $200 \begin{array}{llllllllllllllll}10 & 4 & 4 & 4 & 4 & 4 & 6 & 6 & \text { STEEL } & 2 & \text { PCM } & 2.9 & 50 \% & 16\end{array}$ 3 CR-ERP \#3 102193 MINS 300104444444466 STEEL 1 PCM $2.9100 \% 20$ 4 CR-ERP \#3 102193 MINS 30010444444466 STEEL 3 PCM $2.9100 \% 16$

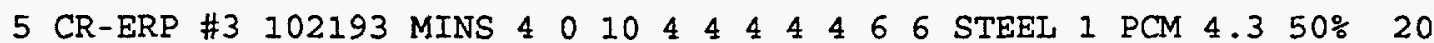
6 CR-ERP \#3 102193 MINS 400104444444466 STEEL 4 PCM 4.3 50\% 16 7 CR-ERP \#3 102193 MINS 50010444444466 STEEL 1 PCM $4.3100 \% 20$ 8 CR-ERP \#3 102193 MINS 500104444444466 STEEL 5 PCM 4.3 100\% 16 9 CR-ERP \#3 102193 MINS 600104444444466 STEEL 1 PCM $5.150 \%$

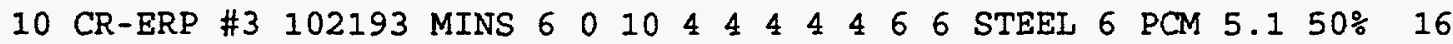
11 CR-ERP \#3 102193 MINS 70010444444466 STEEL 1 PCM 5.1 100\% 18 12 CR-ERP \#3 102193 MINS $70 \begin{array}{llllllllllll}10 & 4 & 4 & 4 & 4 & 4 & 6 & 6 & \text { STEEL } 7 & \text { PCM } 5.1 & 100 \% & 18\end{array}$ 
UNIVARIATE PROCEDURE

Variable $=$ CENTER

Moments

$\begin{array}{lrlr}\text { N } & 28 & \text { Sum Wgts } & 28 \\ \text { Mean } & 0 & \text { Sum } & 0 \\ \text { Std Dev } & 0.089688 & \text { Variance } & 0.008044 \\ \text { Skewness } & -1.93773 & \text { Kurtosis } & 4.74489 \\ \text { USS } & 0.217187 & \text { CSS } & 0.217187 \\ \text { CV } & 0 & \text { Std Mean } & 0.016949 \\ \text { T:Mean=0 } & 0 & \text { Prob }>|\mathrm{T}| & 1.0000 \\ \text { Sgn Rank } & 15 & \text { Prob }>|S| & 0.5880 \\ \text { Num A }=0 & 20 & & \\ \text { W:Normal } & 0.76776 & \text { Prob }<W & 0.0001\end{array}$

Quantiles (Def $=5$ )

$\begin{array}{crrr}100 \% \operatorname{Max} & 0.105215 & 99 \% & 0.105215 \\ 75 \% \text { Q3 } & 0.040743 & 95 \% & 0.105215 \\ 50 \% \text { Med } & 0.040743 & 90 \% & 0.105215 \\ 25 \% \text { Q1 } & 0 & 10 \% & -0.12223 \\ 0 \% \text { Min } & -0.31564 & 5 \% & -0.13579 \\ & & 1 \% & -0.31564\end{array}$

Range $\quad 0.42086$

Q3-Q1 $\quad 0.040743$

Mode 0

Extremes

$\begin{array}{rrrr}\text { Iowest } & \text { Obs } & \text { Highest } & \text { Obs } \\ -0.31564( & 54) & 0.045264( & 32) \\ -0.13579( & 34) & 0.045264( & 33) \\ -0.12223( & 14) & 0.105215( & 51) \\ -0.12223( & 43) & 0.105215( & 52) \\ -0.12223( & 21) & 0.105215( & 53)\end{array}$

Missing Value

Count

42

$\because$ Count/Nobs

60.00 
$\because \quad$-Tests of assumptions for survival data (arcsine transformed)
TEST FOR NORMAL DISTRIBUTION

20:32 Sunday, October 27,1991

\section{STUDY $=C R-E R P \quad \# 3$ ST_DATE $=102193 \quad$ PARM=MINS}

\section{UNIVARIATE PROCEDURE}

Variable $=$ CENTER
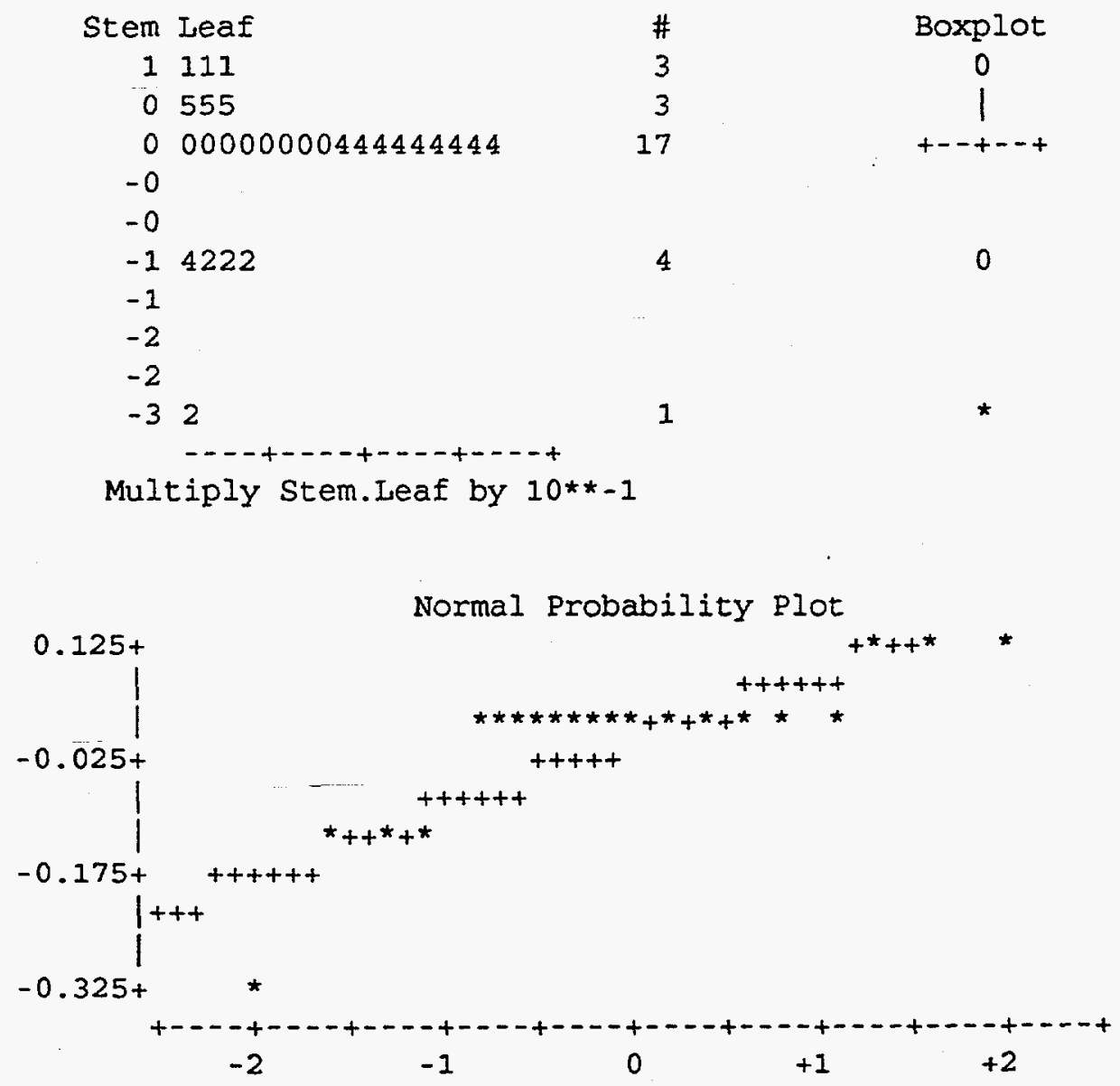
$\because \quad \cdot \quad$ Tests of assumptions for survival data (arcsine transformed)
TEST FOR NORMAL DISTRIBUTION

20:32 Sunday, October 27, 1991

UNIVARIATE PROCEDURE

Schematic Plots

Variable $=$ CENTER

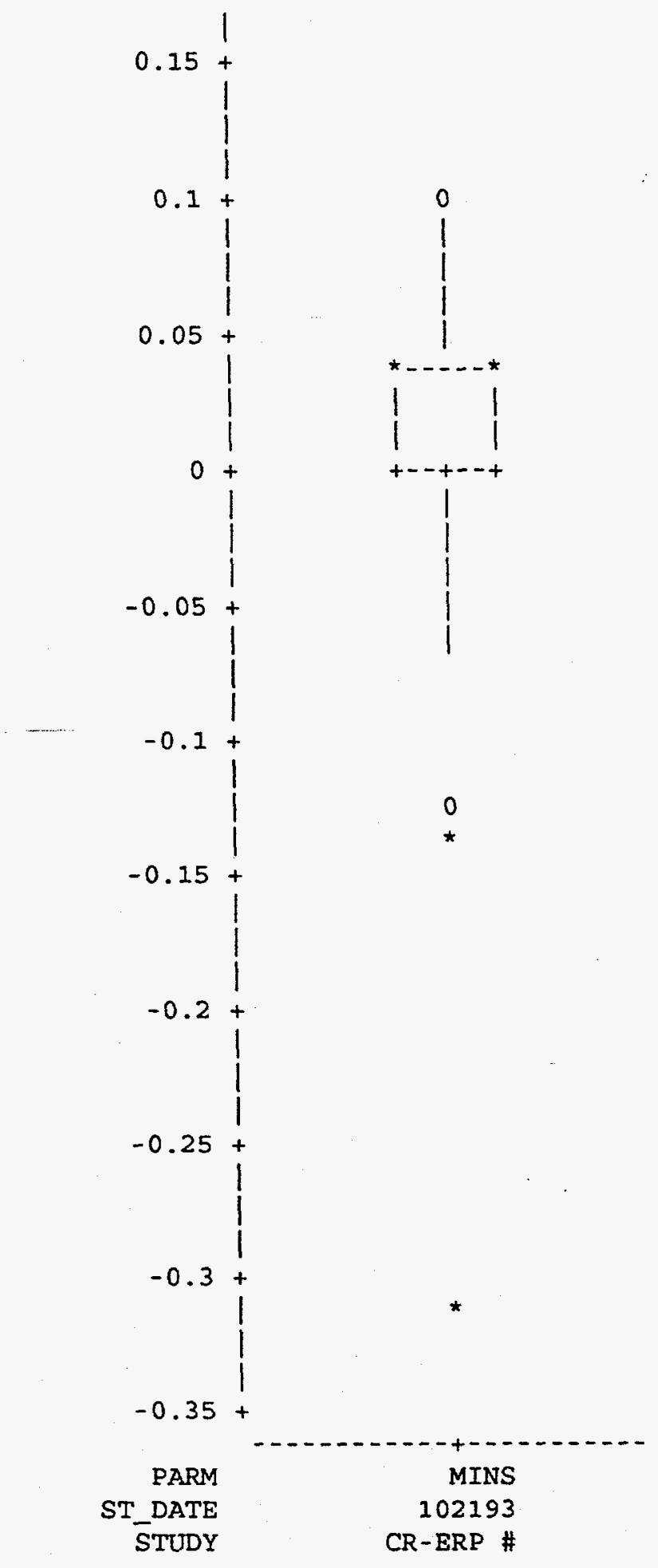


Plot of CENTER*RANKIT. Symbol is value of TRT_NO.

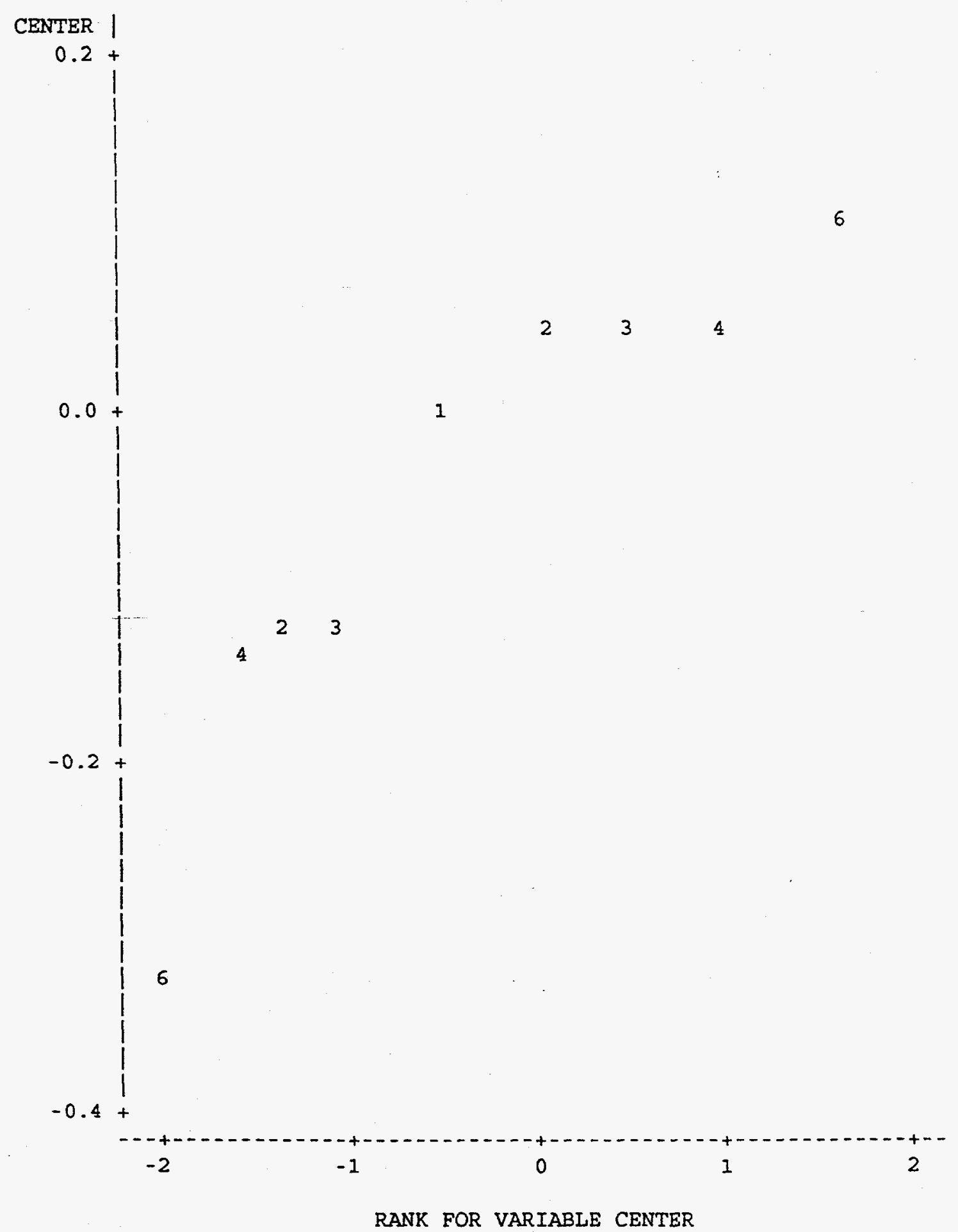

NOTE: 42 obs had missing values. 19 obs hidden. 
$\because$ i Tests of assumptions for survival data (arcsine transformed)

\section{FREQUENCY OF CENTER}

\section{FREQUENCY}

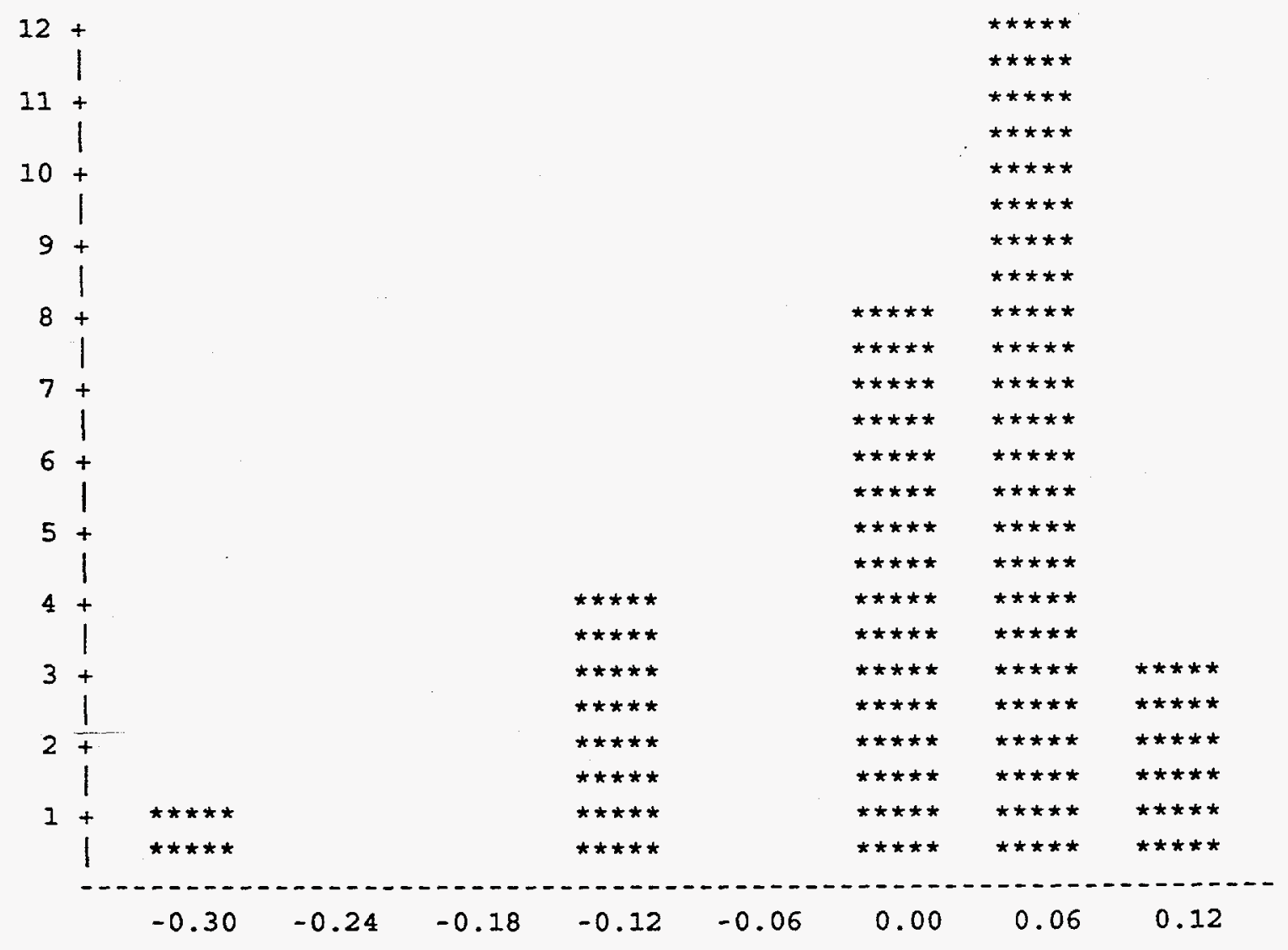

CENTER MIDPOINT 
20:32 Sunday, October 27, 1991

VARIANCE EQUAL TO ZERO FOR ONE OR MORE TREATMENTS

BARTLETT'S TEST CAN NOT BE CALCULATED

LEVENE'S TEST WIIL BE USED 


$$
\begin{aligned}
\because \quad . T e s t s \text { of assumptions for survival data (arcsine transformed) } & 7 \\
\text { LEVENE'S TEST FOR HOMOGENEITY OF VARIANCE } & \\
& 20: 32 \text { Sunday, October 27, } 1991
\end{aligned}
$$

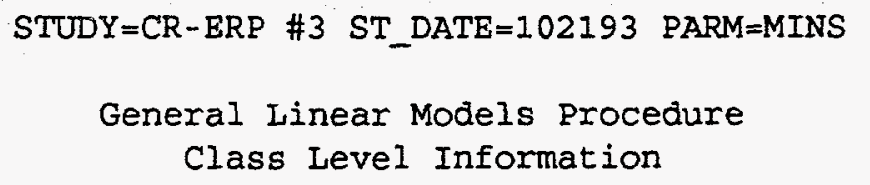

NOTE: Due to missing values, only 28 observations can be used in this analysis. 
$\because \quad$ Tests of assumptions for survival data (arcsine transformed) LEVENE'S TEST FOR HOMOGENEITY OF VARIANCE

$S T U D Y=C R-E R P \quad \# 3 \quad S T \_D A T E=102193 \quad$ PARM=MINS

General Linear Models Procedure

Dependent Variable: ABS_VAL

\begin{tabular}{|c|c|c|c|c|c|c|}
\hline Source & DF & $\begin{array}{l}\text { Sum of } \\
\text { Squares }\end{array}$ & $\begin{array}{r}\text { Mean } \\
\text { Square }\end{array}$ & F & Value & $\operatorname{Pr}>F$ \\
\hline Model & 6 & 0.06727273 & 0.01121212 & & 4.34 & 0.0053 \\
\hline Error & 21 & 0.05429667 & 0.00258556 & & & \\
\hline Corrected Total & 27 & 0.12156941 & & & & \\
\hline & R-Square & C.V. & Root MSE & \multicolumn{3}{|c|}{ ABS_VAL Mean } \\
\hline & 0.553369 & 87.01373 & 0.050848 & \multicolumn{3}{|c|}{0.05843717} \\
\hline Source & DF & Type I SS & Mean Square & $\mathrm{F}$ & Value & $\operatorname{Pr}>F$ \\
\hline TRT & 6 & 0.06727273 & 0.01121212 & & 4.34 & 0.0053 \\
\hline Source & DF & Type III SS & Mean Square & $\mathbf{F}$ & value & $\operatorname{Pr}>F$ \\
\hline - २T & 6 & 0.06727273 & 0.01121212 & & 4.34 & 0.0053 \\
\hline
\end{tabular}


Study/Date CR-ERP Cet 23

FltarLo dRI IBIGH RBCORD SABBT

Page 1 of 2

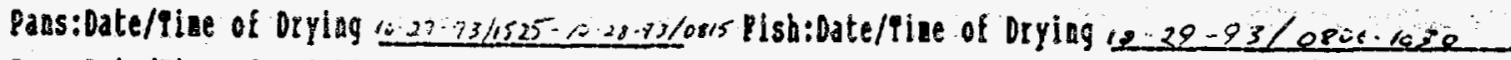

Personnel os

Pans:Date/fine of Ielghing $10.25: 3 / 12$ ou 1312 Pish:Date/fine of Ielghing $0.29 \cdot 23 / 1215 \cdot 1450$

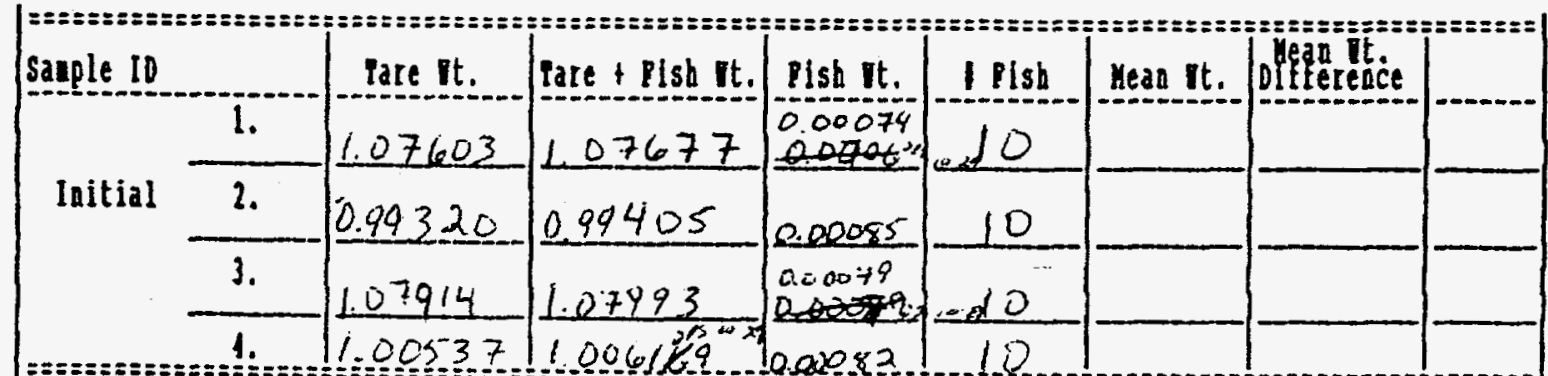

Injial it.

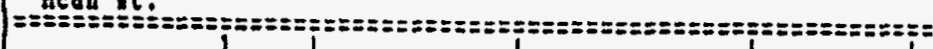

E.MED $\frac{1 .}{2 .}\left|\frac{1.02634}{1.03445}\right| \frac{1.03145}{1.03715}$

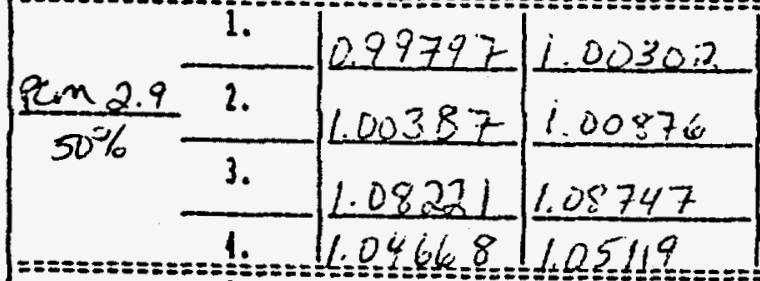

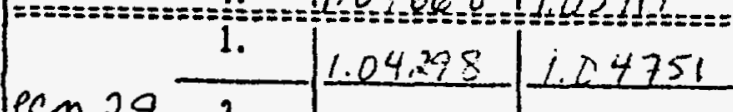

$\frac{\operatorname{lem} 29}{100 \%-\frac{1 .}{3 .}} \frac{1.03796}{104278}$

$\frac{100989}{1} 1.01493$

: $=:=079631.085 x^{\prime 4}$

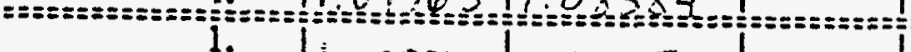

\begin{tabular}{l}
10 \\
\hline 10 \\
\hline 10
\end{tabular}

Pem $4.32 .01 .0327 ! 1.03553$

$50^{2} \mathrm{~L}-3$.

3.

1.02576

1.0 .160

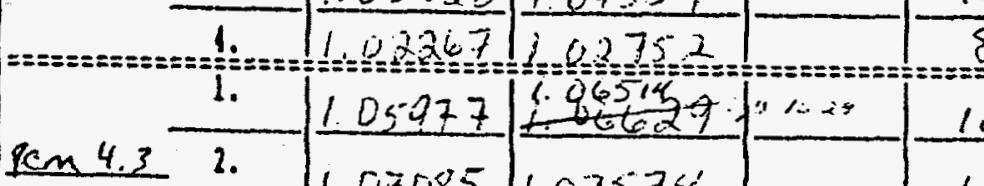

$100 \%$

3.

1. $\frac{.07328}{0.032} \frac{1.03263}{1.0219}$

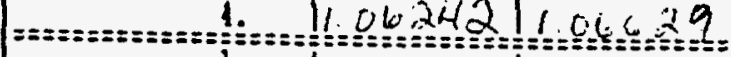

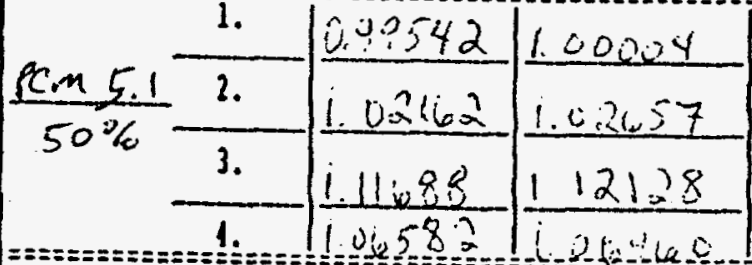

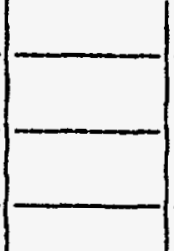

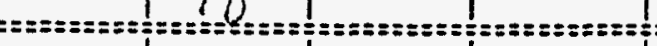

$\prod_{i=2:=2=}^{1}$

Motes:

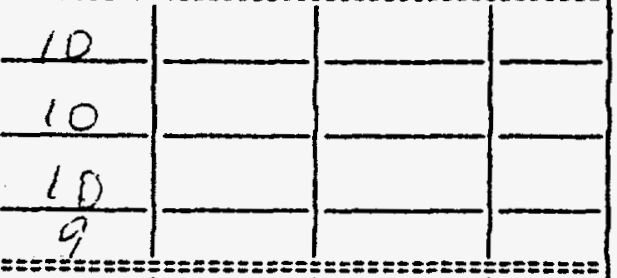

$\longrightarrow$

$\frac{9}{10}-\square$

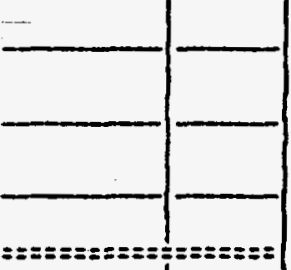

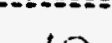

$\frac{10}{10}$

|

Revieved By: _ Cure 
Stady/Date $\quad$ CR-ERP OXI 93

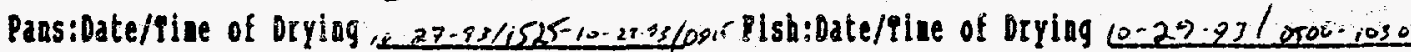

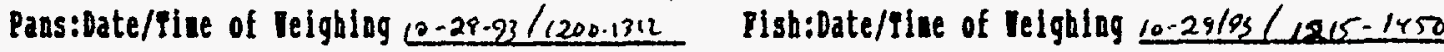

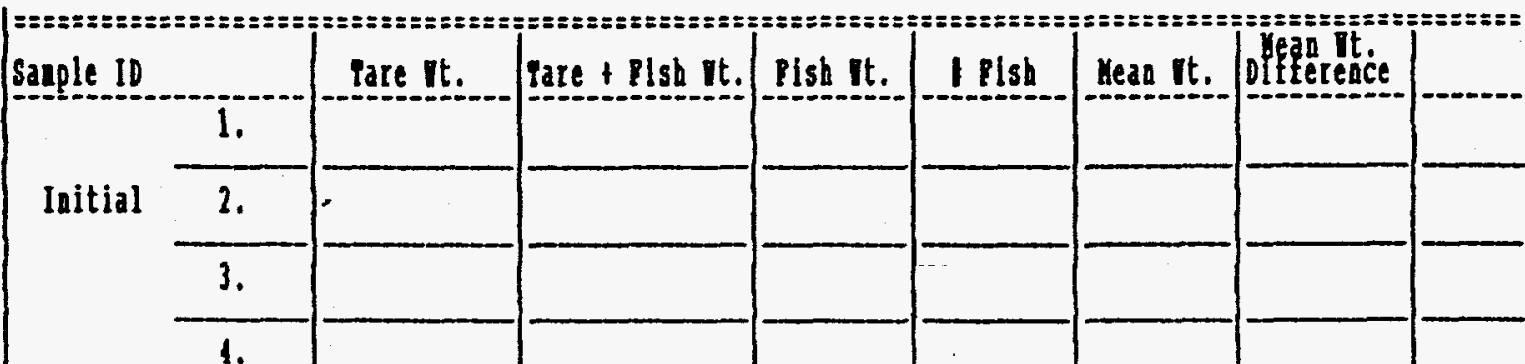

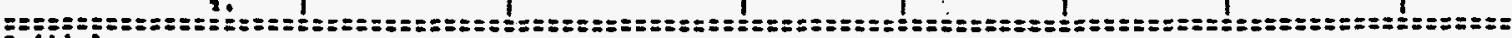
Injtial leañ It.

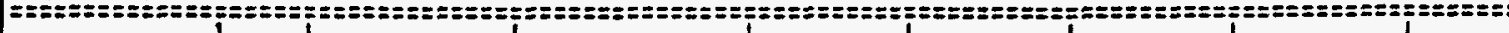

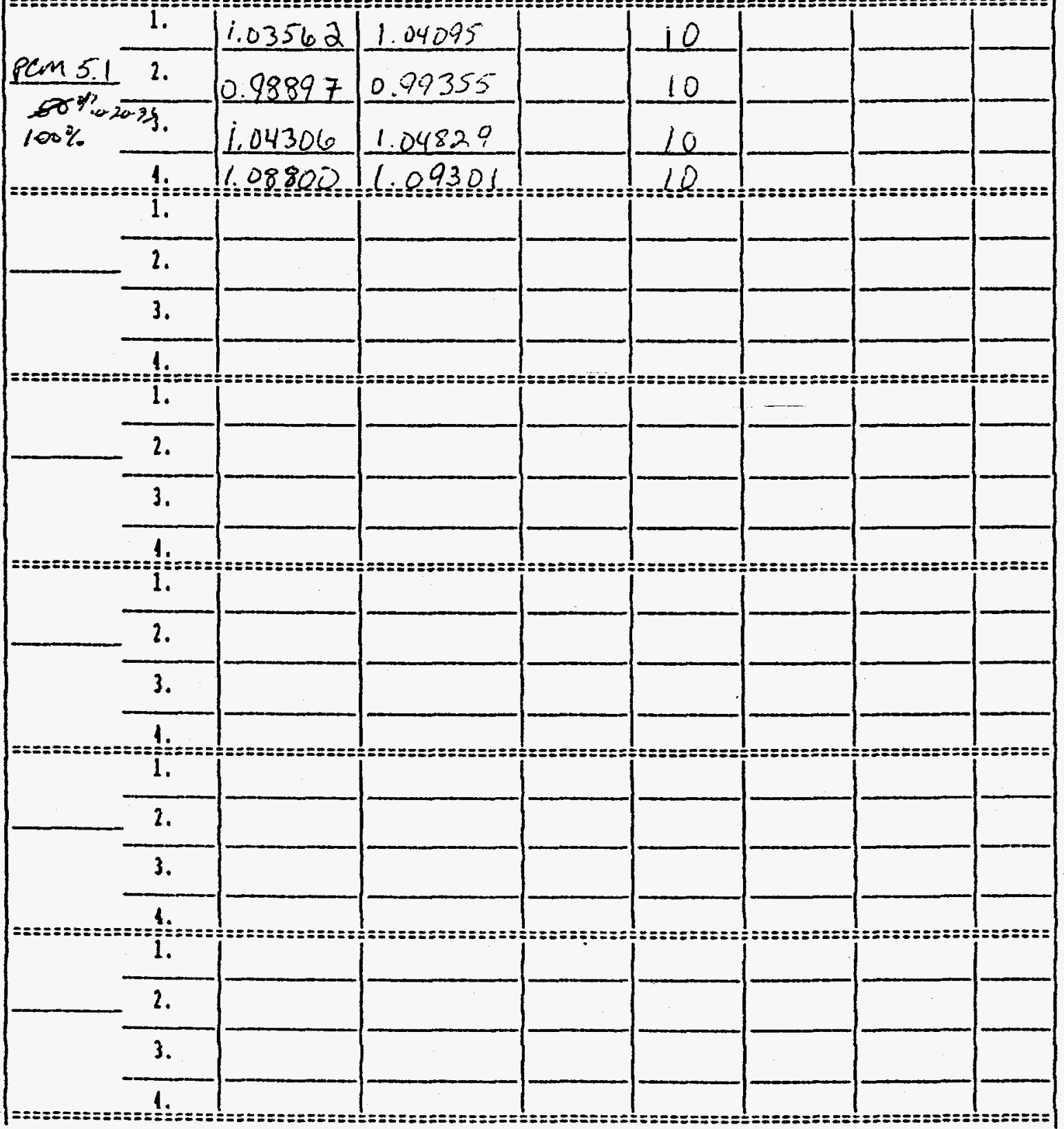


Stody/Date ERede QL Oet 93 Reweorl Pans:Date/tine of Orying i1/4193/0.33-3846

Pish:Date/fine of Drying ofy/43/inas-1200 Pans: Date/tine of leighing a/y/az/108-100

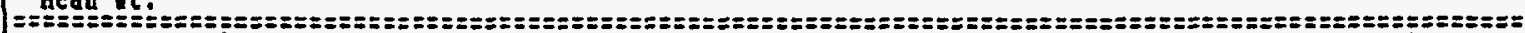

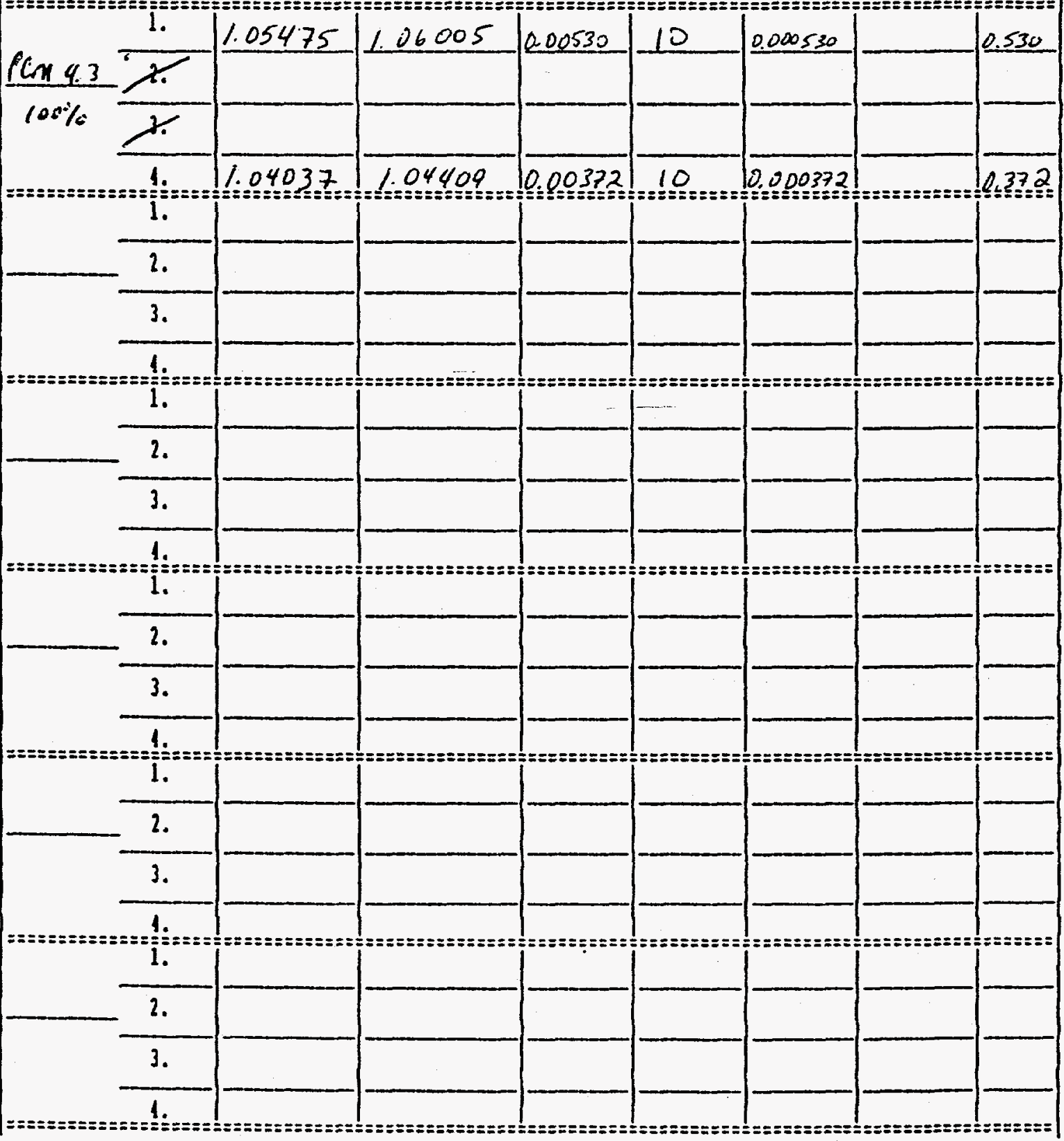

Motes: 


\section{FATHEAD DRY WEIGHT RECORD SHEET}

Page 1 of 2

Study/Date: DOE /3

Perbonnel: DJS

Pans:Date/Time of Drying: 10/27/93-1525 to 10/28/93-0815

Pans:Date/Time of Keighing: $10-28-93 / 1200$

Pish:Dace/Time of Drying: 10-29-93/0800

Pish:Date/Time of Weighing: 10-29-93/1215

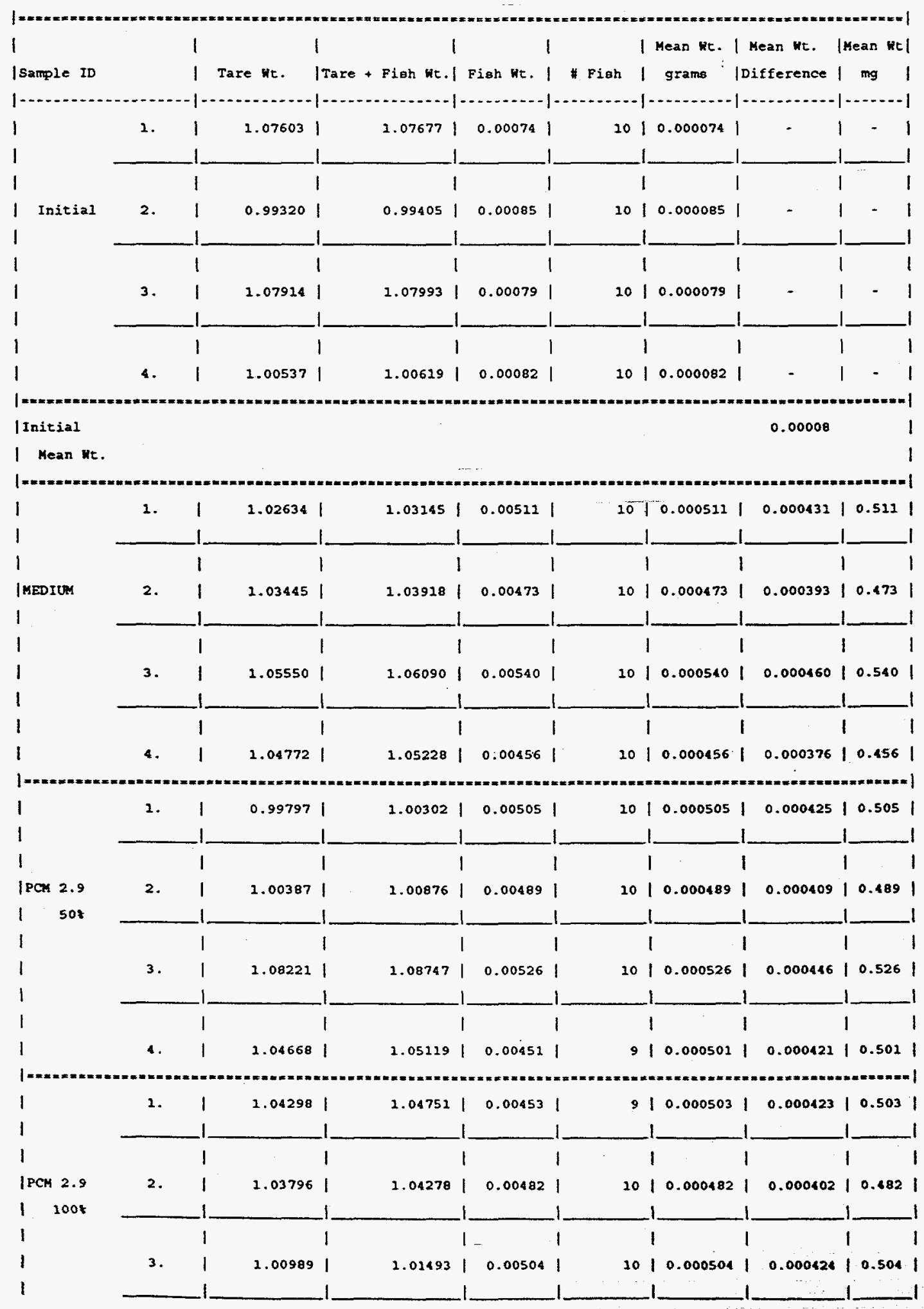

Notee: 

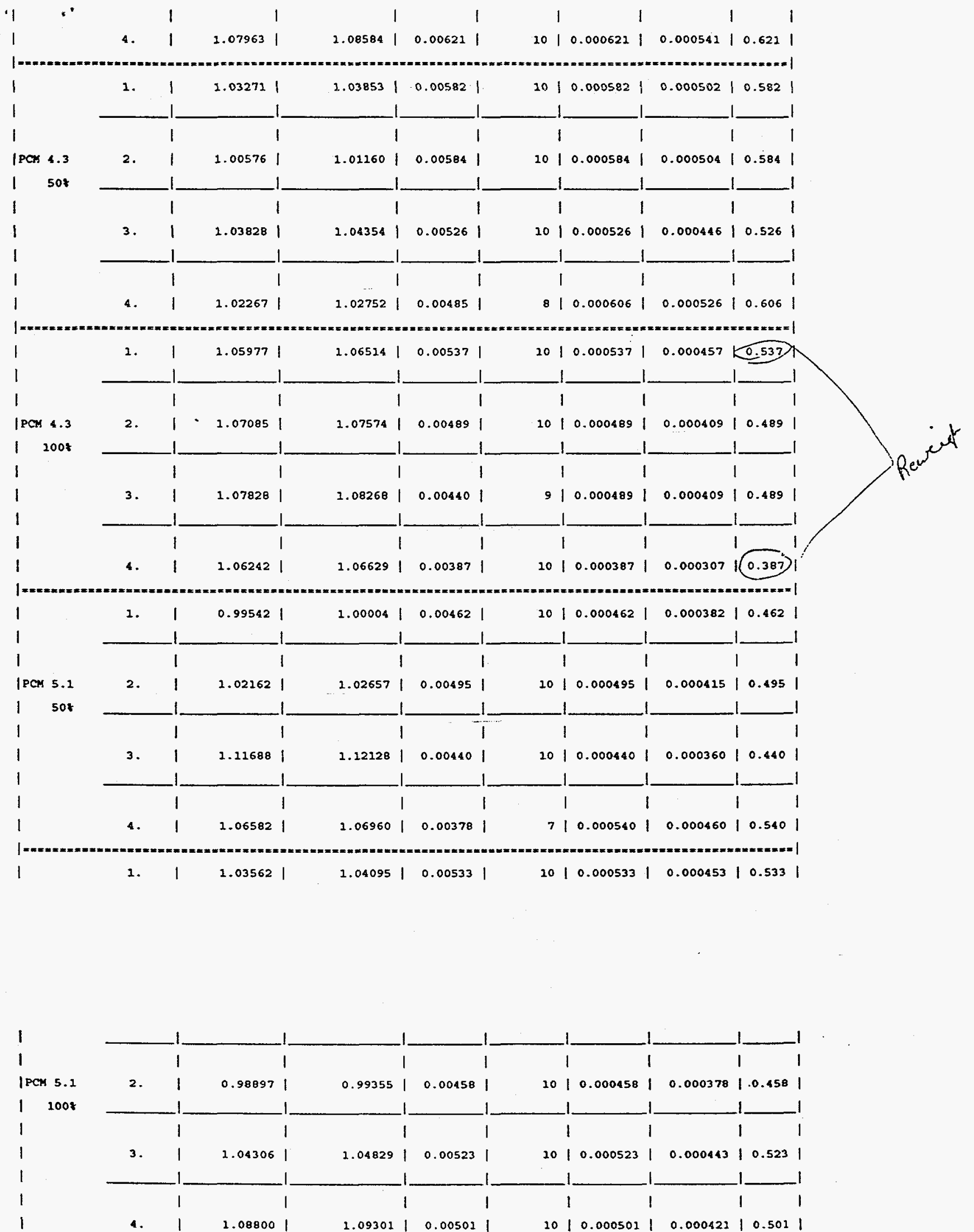

|

Reviewed By: Rusaell

$$
11-0.53
$$


Analysis of 7-day Larval Growth Test

CR-ERP \#3

Starting Date: $10 / 21 / 93$

Analysis of Dry Weight (mg) Data Dunnett's Test

No transformation applied before data analysis.

For this set of data, the minimum significant difference is 0.08

This represents a $15.51 \%$ reduction in Dry Weight (mg).

$$
T=2.45 \text { ALPHA }=0.05
$$

Dry Weight (mg) Data

Replicate

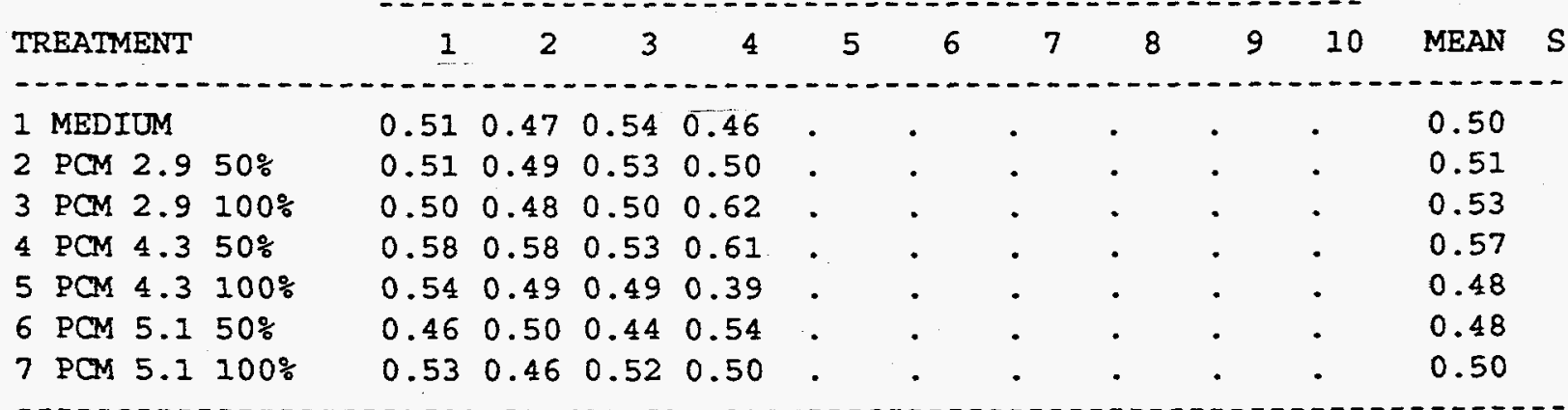

Asterisk $(*)$ indicates values significantly less than control (MEDIUM).

Analysis of variance

Source DF Sum OF Sq. Mean Sq. Calc $F$ F(0.05)

\begin{tabular}{|c|c|c|c|c|}
\hline Among & 6 & 0.026 & 0.004 & 2.180 \\
\hline Within & 21 & 0.041 & 0.002 & \\
\hline
\end{tabular}

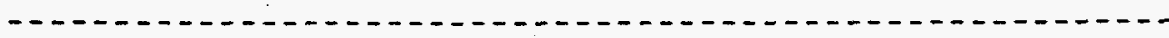

Total $27 \quad 0.000$ 
Analysis of untransformed data 1

20:56 Sunday, October 27, 1991

STUDY $=C R-E R P \quad \# 3$

ST DATE $=102193$

PARM $=$ MING

DUNNETT'S TEST

$T=2.45$ ALPHA $=0.05$

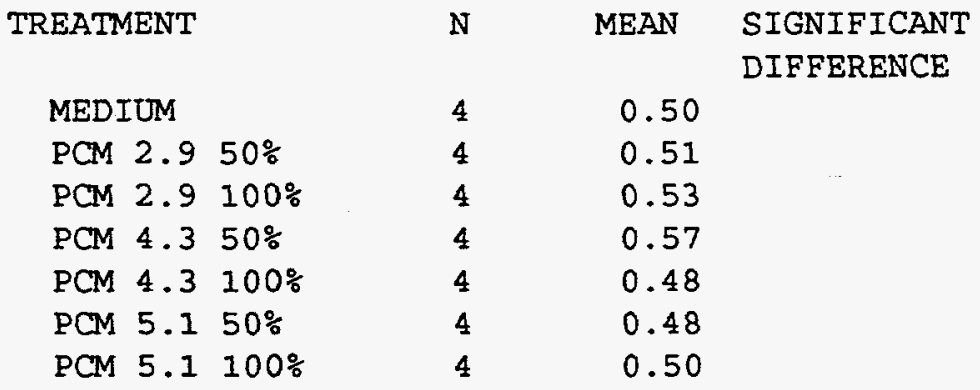

MINIMUM SIGNIFINANT DIFFERENCE $=0.08$

THIS REPRESENTS A 15.51 DECREASE IN SURVIVAL FROM THE CONTROL 
STUDY=CR-ERP \#3

ST_DATE $=102193$

PARM=MING

DUNNETT'S TEST

$T=2.45$ ALPHA $=0.05$

$123 \quad$ REPLICATE

1 MEDIUM

2 PCM $2.950 \%$

3 PCM $2.9100 \%$

4 PCM $4.350 \%$

$5 \mathrm{PCM} 4.3100 \%$

6 PCM $5.150 \%$

7 PCM $5.1100 \%$

$\begin{array}{llll}0.51 & 0.47 & 0.54 & 0.46 \\ 0.51 & 0.49 & 0.53 & 0.50 \\ 0.50 & 0.48 & 0.50 & 0.62 \\ 0.58 & 0.58 & 0.53 & 0.61 \\ 0.54 & 0.49 & 0.49 & 0.39 \\ 0.46 & 0.50 & 0.44 & 0.54 \\ 0.53 & 0.46 & 0.52 & 0.50\end{array}$

6

$\begin{array}{llllll}7 & 8 & 9 & 10 & \text { MEAN } & S\end{array}$

MINIMUM SIGNIFINANT DIFFERENCE $=0.08$ THIS REPRESENTS A $15.51 \%$ DECREASE IN SURVIVAL FROM THE CONTROL 


$$
\begin{array}{r}
\text { Analysis of untransformed data } \\
20: 56 \text { Sunday, October 27, } 1991
\end{array}
$$

General Linear Models Procedure

Class Level Information

Class Levels Values

TRT

7 MEDIUM PCM $2.9100 \%$ PCM $2.950 \%$ PCM $4.3 \quad 100 \%$ PCM $4.350 \%$

PCM $5.1100 \%$ PCM $5.150 \%$

Number of observations in data set $=70$

NOTE: Due to missing values, only 28 observations can be used in this analysis. 
20:56 Sunday, October 27, 1991

General Linear Models Procedure

Dependent Variable: RESP

Source DF

Model

Error

Corrected Total

R-Square

0.383749

Source

TRT

Source

TRT
DF

6

DF

6
Sum of

Squares

0.02567143

0.04122500

0.06689643

C.V.

8.693702

Type I SS

0.02567143

Type III SS

0.02567143
Mean

Square

0.00427857

2.18

0.0863

0.00196310

Root MSE

RESP Mean

0.0443068

0.50964286

Mean Square

0.00427857

2.18

0.0863

Mean Square

F Value

0.00427857

2.18

0.0863 
Test for Normal Distribution

\section{STUDY=CR -ERP \#3 ST_DATE=102193 PARM=MING}

UNIVARIATE PROCEDURE

Variable $=$ CENTER

Moments

$\begin{array}{lrlr}\text { N } & 28 & \text { Sum Wgts } & 28 \\ \text { Mean } & 0 & \text { Sum } & 0 \\ \text { Std Dev } & 0.039075 & \text { Variance } & 0.001527 \\ \text { Skewness } & 0.213347 & \text { Xurtosis } & 0.403796 \\ \text { USS } & 0.041225 & \text { CSS } & 0.041225 \\ \text { CV } & . & \text { Std Mean } & 0.007384 \\ \text { T:Mean=0 } & 0 & \text { Prob }>|T| & 1.0000 \\ \text { Sgn Rank } & -3.5 & \text { Prob }>|S| & 0.9382 \\ \text { Num A }=0 & 28 & & \\ \text { W: Normal } & 0.982811 & \text { Prob<W } & 0.9142\end{array}$

Quantiles (Def=5)

$\begin{array}{lrrr}100 \% \text { Max } & 0.095 & 99 \% & 0.095 \\ 75 \% \mathrm{Q3} & 0.02 & 95 \% & 0.0625 \\ 50 \% \text { Med } & 0.00375 & 90 \% & 0.055 \\ 25 \% \mathrm{Q} 1 & -0.025 & 10 \% & -0.045 \\ 0 \% \text { Min } & -0.0875 & 5 \% & -0.045 \\ & & 1 \% & -0.0875 \\ \text { Range } & 0.1825 & & \\ \text { Q3-Q1 } & 0.045 & & \\ \text { Mode } & -0.025 & & \end{array}$

Extremes

$\begin{array}{lrrr}\text { Lowest } & \text { Obs } & \text { Highest } & \text { Obs } \\ -0.0875( & 44) & 0.035( & 34) \\ -0.045( & 22) & 0.045( & 3) \\ -0.045( & 53) & 0.055( & 54) \\ -0.045( & 33) & 0.0625( & 41) \\ -0.0425( & 62) & 0.095( & 24)\end{array}$

Missing Value

Count 42

$\because$ Count/Nobs $\quad 60.00$ 
Test for Normal Distribution

20:50 Sunday, October 27, 1991

\section{UNIVARIATE PROCEDURE}

Variable $=$ CENTER
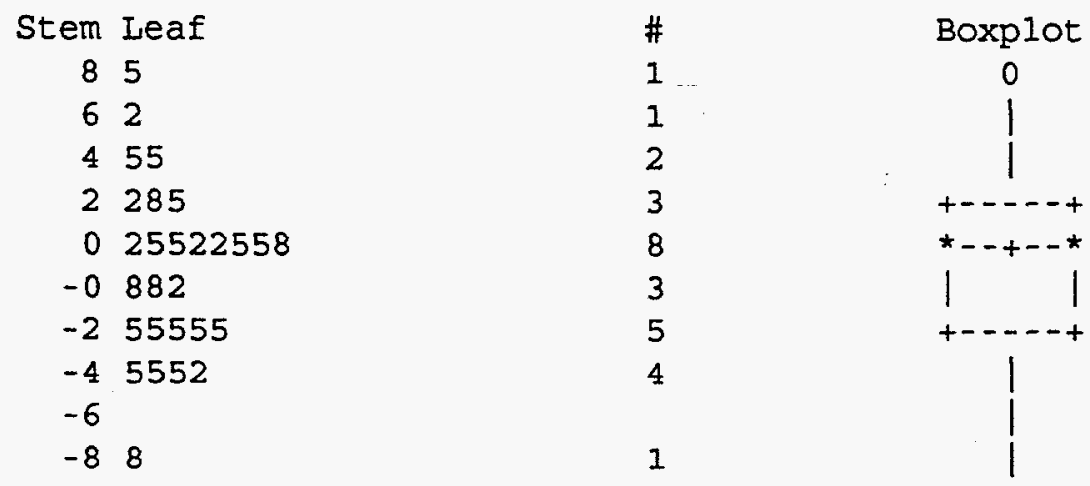

Multiply Stem.Leaf by $10 * \star-2$

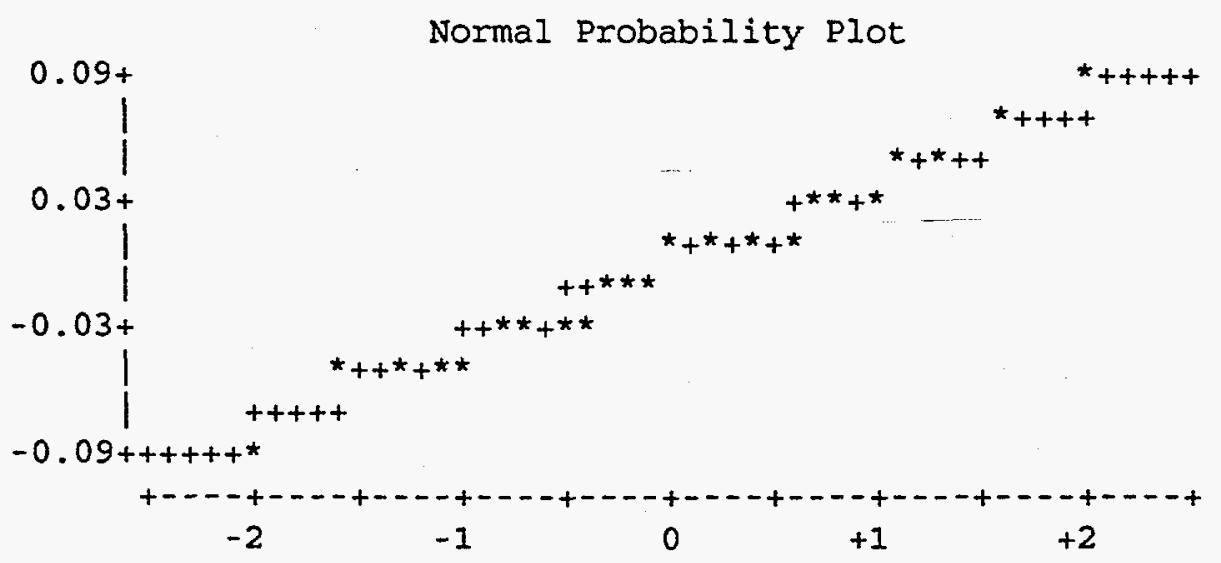


Tests of assumptions for untransformed data Test for Normal Distribution

20:50 Sunday, October 27, 1991

UNIVARIATE PROCEDURE

Schematic Plots

Variable $=$ CENTER

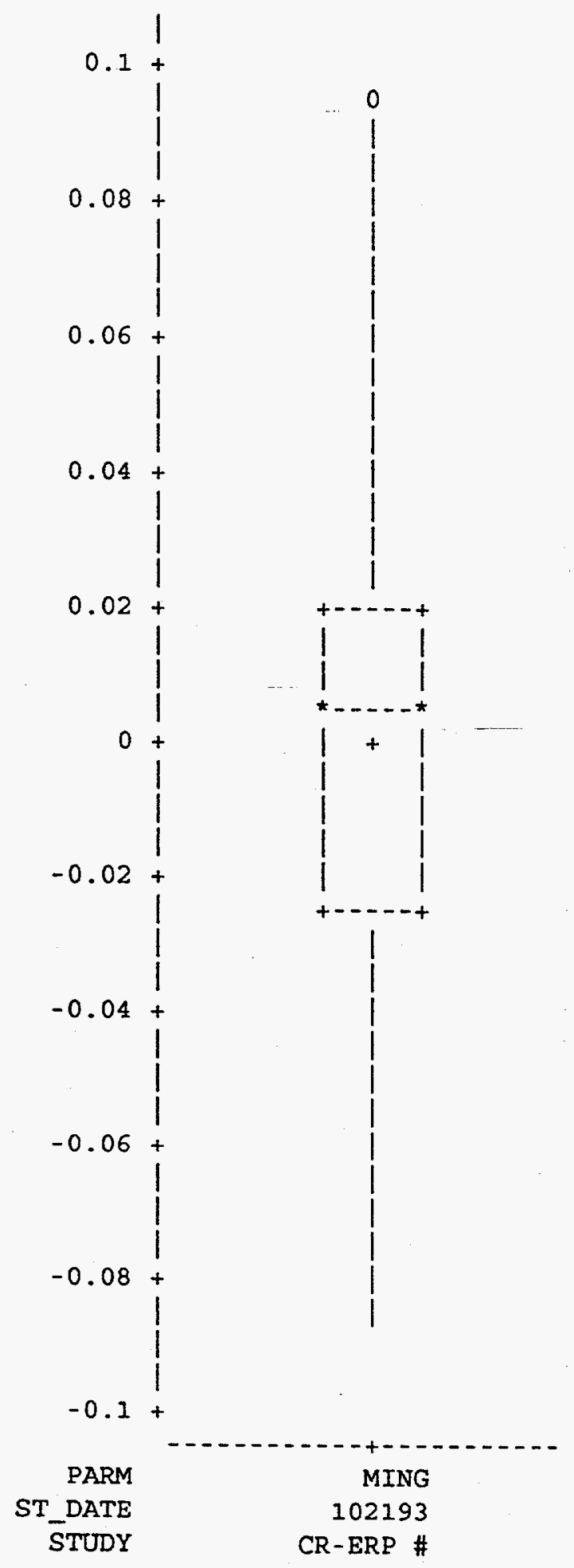


Plot of CENTER*RANKIT. Symbol is value of TRT_NO.

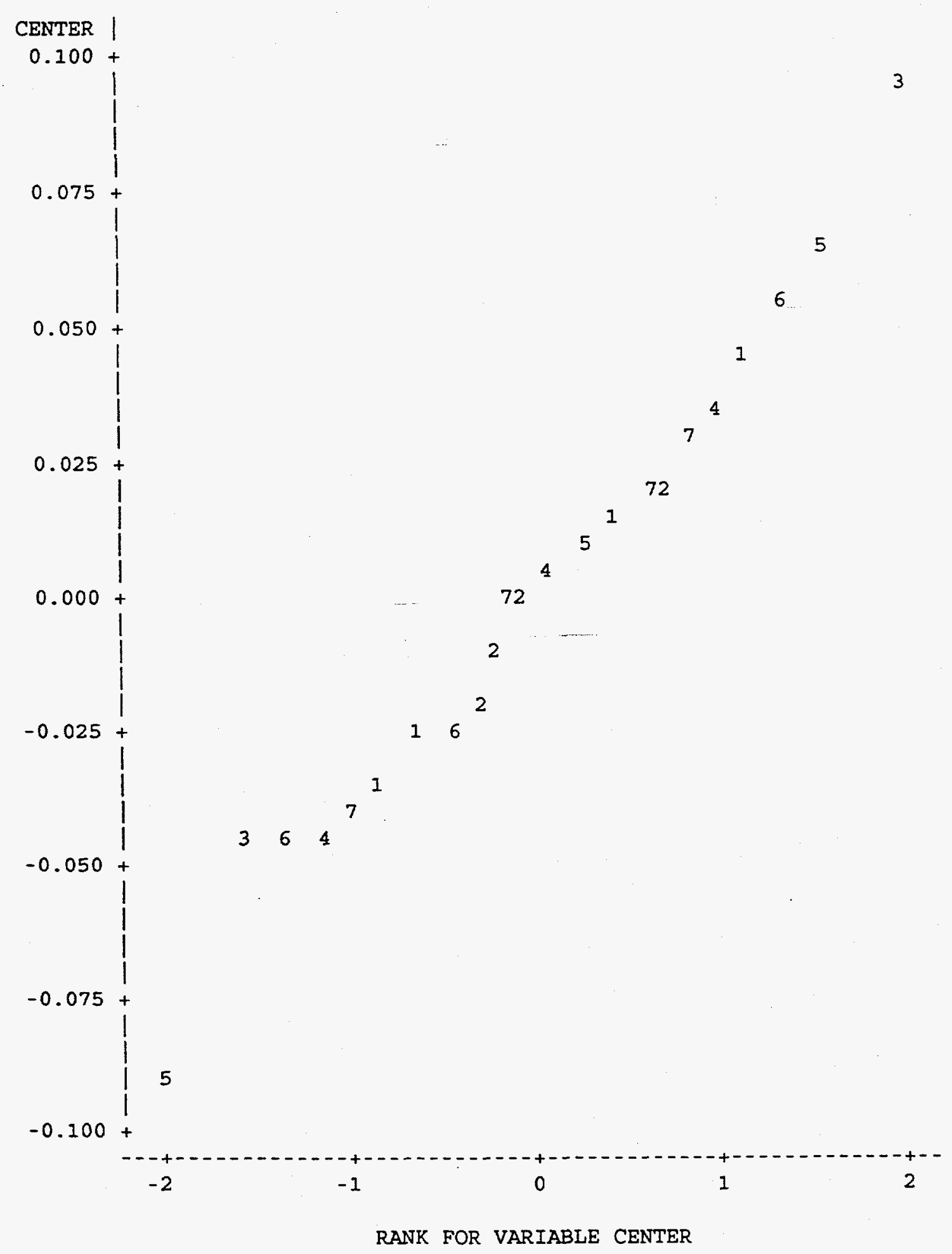

NOTE: 42 obs had missing values. 5 obs hidden. 
$S T U D Y=C R-E R P \# 3$

ST_DATE $=102193$

PARM $=$ MING

$\begin{array}{lllll}\text { TREATMENT } & \text { N } & \text { MEAN } & \text { VARIANCE } \\ \text { MEDIUM } & \ldots & 0.50 & 0.001 \\ \text { PCM } 2.95 & 4 & 0.51 & 0.000 \\ \text { PCM 2.9 } & 4 & 4 & 0.53 & 0.004 \\ \text { PCM } 4.35 & 4 & 0.57 & 0.001 \\ \text { PCM } 4.31 & 4 & 0.48 & 0.004 \\ \text { PCM 5.1 } 5 & 5 & 4 & 0.48 & 0.002 \\ \text { PCM 5.1 } & 4 & 4 & 0.50 & 0.001\end{array}$

BARTLETT'S TEST STATISTIC $=5.658$

$\mathrm{PROB}>\mathrm{B}=0.463$ 
CR-ERP \#

CR-ERP \#3

-ERP \#3

CR-ERP \#3

CR-ERP \#3

CR-ERP \#3

CR-ERP \#3
102193 MINS

INITIAL NUMBER

102193 MINS

INITIAL NUMBER

102193 MINS

INITIAL NUMBER

102193 MINS

INITIAI NUMBER

102193 MINS

INITIAL NUMBER

102193 MINS

INITIAL NUMBER

102193 MINS

INITIAL NUMBER
1 MEDIUM

2 PCM $2.950 \%$

3 PCM $2.9100 \%$

4 PCM $4.350 \%$

5 PCM $4.3 \quad 100 \%$

6 PCM $5.150 \%$

7 PCM $5.1 \quad 100 \%$

$\begin{array}{rrrr}10.00 & 10.00 & 10.00 & 10.00 \\ 10.00 & 10.00 & 10.00 & 10.00 \\ 10.00 & 10.00 & 10.00 & 9.00 \\ 10.00 & 10.00 & 10.00 & 10.00 \\ 9.00 & 10.00 & 10.00 & 10.00 \\ 10.00 & 10.00 & 10.00 & 10.00 \\ 10.00 & 10.00 & 10.00 & 8.00 \\ 10.00 & 10.00 & 10.00 & 9.00 \\ 10.00 & 10.00 & 9.00 & 10.00 \\ 10.00 & 10.00 & 10.00 & 10.00 \\ 10.00 & 10.00 & 10.00 & 7.00 \\ 10.00 & 10.00 & 10.00 & 10.00 \\ 10.00 & 10.00 & 10.00 & 10.00 \\ 10.00 & 10.00 & 10.00 & 10.00\end{array}$


$C R-$ ERP \#3 CR-ERP \#3 CR-ERP \#3 CO-ERP \#3 -ERP \#3 CR-ERP \#3 CR-ERP \#3
102193 MING 1 MEDIUM

102193 MING 2 PCM $2.950 \%$

102193 MING 3 PCM $2.9100 \%$

102193 MING 4 PCM $4.350 \%$

102193 MING 5 PCM $4.3100 \%$

102193 MING 6 PCM $5.150 \%$

102193 MING 7 PCM $5.1100 \%$

$\begin{array}{llll}0.51 & 0.47 & 0.54 & 0.46 \\ 0.51 & 0.49 & 0.53 & 0.50 \\ 0.50 & 0.48 & 0.50 & 0.62 \\ 0.58 & 0.58 & 0.53 & 0.61 \\ 0.54 & 0.49 & 0.49 & 0.39 \\ 0.46 & 0.50 & 0.44 & 0.54 \\ 0.53 & 0.46 & 0.52 & 0.50\end{array}$

0.46

0.61

0.50 
CR - ERP - P C (POplar Creek) -- OCTOBER 21-28, 1993 -- INITIAL AND FINAL CHEMISTRY

\section{INITIAL CHENISTRY}

FISH MEDIUM

\begin{tabular}{llllllll}
\multicolumn{7}{c}{ INITIAL WARMED } \\
OAY & TEMP & TEMP & DO & PH & COND & HARD & ALK CHLORINE \\
0 & 22.9 & 25.0 & 8.3 & 8.2 & 360 & 94.1 & 68 \\
1 & 21.6 & 25.1 & 8.4 & 8.2 & 351 & & \\
2 & 22.2 & 25.2 & 8.4 & 8.2 & 351 & & \\
3 & 22.7 & 25.3 & 8.4 & 8.2 & 353 & & \\
4 & 23.3 & 25.0 & 8.3 & 8.2 & 338 & 94.1 & 65 \\
5 & 23.7 & 25.3 & 8.2 & 8.3 & 339 & & \\
6 & 25.2 & 25.4 & 8.1 & 8.2 & 342 & & \\
MEAN & 23.1 & 25.2 & 8.3 & 8.2 & 348 & 94.1 & 67 \\
& & & & & & & \\
MIN & 21.6 & 25.0 & 8.1 & 8.2 & 338 & 94.1 & 65 \\
MAX & 25.2 & 25.4 & 8.4 & 8.3 & 360 & 94.1 & 68
\end{tabular}

PCM $2.9-50 \%$

\begin{tabular}{|c|c|c|c|c|c|}
\hline \multicolumn{6}{|c|}{ INITIAL WARMEO } \\
\hline DAY & TEMP & IEMP & DO & $\mathrm{pH}$ & COND \\
\hline 0 & & 25.0 & 8.4 & 7.9 & 366 \\
\hline 1 & & 24.7 & 8.4 & 8.0 & 353 \\
\hline 2 & & 25.3 & 8.2 & 8.0 & 364 \\
\hline 3 & & 25.3 & 8.2 & 8.0 & 363 \\
\hline 4 & & 25.0 & 8.4 & 8.0 & 355 \\
\hline 5 & & 25.4 & 8.2 & 8.0 & 358 \\
\hline 6 & & 25.6 & 8.3 & 7.1 & 361 \\
\hline MEAK & & 25.2 & 8.3 & 7.9 & 360 \\
\hline IIN & & 24.7 & 8.2 & 7.1 & 353 \\
\hline KaX & & 25.6 & 8.4 & 8.0 & 66 \\
\hline
\end{tabular}

PCM $2.9-100 \%$

INITIAL WARMED

DAY TEKP TENP DO PH CONO HARD ALK CHLORINE

$\begin{array}{lllllllll}0 & 1.3 & 25.1 & 8.4 & 7.5 & 369 & 153.9 & 113 & 10.1\end{array}$

$\begin{array}{lllllll}1 & 3.3 & 24.7 & 8.4 & 8.0 & 363\end{array}$

$\begin{array}{llllllllll}2 & 0.5 & 25.3 & 8.0 & 7.7 & 378 & 153.9 & 117 & 10.1\end{array}$

$\begin{array}{llllll}3 & 3.2 & 25.1 & 8.3 & 7.7 & 378\end{array}$

$\begin{array}{llllll}4 & 3.5 & 24.9 & 8.4 & 7.9 & 375\end{array}$

$\begin{array}{llllllllll}5 & 0.7 & 25.2 & 8.4 & 7.7 & 381 & 153.9 & 119 & 10.1\end{array}$

$\begin{array}{llllll}6 & 3.0 & 26.0 & 8.4 & 7.6 & 382\end{array}$

$\begin{array}{lllllllll}\text { MEAN } & 2.2 & 25.2 & 8.3 & 7.7 & 375 & 153.9 & 116 & 10.1\end{array}$

MIN $\begin{array}{llllllll}0.5 & 24.7 & 8.0 & 7.5 & 363 & 153.9 & 113 & 10.1\end{array}$

MAX
HARD ALK CHLORINE

PCM $4.3-100 \%$

\section{INITIAL WARMED}

DAY TEMP TEMP DO PH CONO HARO ALK CHLORINE

$\begin{array}{lllllllll}0 & 1.5 & 25.4 & 7.7 & 7.3 & 416 & 162.5 & 120 & 10.1\end{array}$

$\begin{array}{llllll}1 & 3.3 & 24.9 & 8.1 & 7.4 & 413\end{array}$

$\begin{array}{lllllllll}2 & 0.4 & 25.1 & 7.0 & 7.5 & 403 & 159.0 & 120 & 10.1\end{array}$

$\begin{array}{llllll}3 & 3.1 & 25.1 & 7.4 & 7.5 & 404\end{array}$

$\begin{array}{llllll}4 & 3.0 & 24.9 & 7.8 & 7.5 & 404\end{array}$

$\begin{array}{lllllllll}5 & 0.9 & 25.1 & 7.3 & 7.5 & 401 & 159.0 & 126 & 10.1\end{array}$

$\begin{array}{llllll}6 & 2.7 & 25.6 & 7.7 & 7.6 & 403\end{array}$

$\begin{array}{lllllllll}\text { MEAN } & 2.1 & 25.2 & 7.6 & 7.5 & 406 & 160.2 & 122 & 10.1\end{array}$

MIN $\quad \begin{array}{llllllll}0.4 & 24.9 & 7.0 & 7.3 & 401 & 159.0 & 120 & 10.1\end{array}$

$\begin{array}{lllllllll}\text { MAX } & 3.3 & 25.6 & 8.1 & 7.6 & 416 & 162.5 & 126 & 10.1\end{array}$

PCM $5.1-50 \%$

INITIAL WARMED

DAY TEMP TEMP DO PH COND HARO ALK CHLORINE

$\begin{array}{lllll}0 & 24.9 & 8.2 & 7.6 & 375 \\ 1 & 24.6 & 8.4 & 7.8 & 375\end{array}$

$\begin{array}{llllll}1 & 24.6 & 8.4 & 7.8 & 375\end{array}$

$\begin{array}{llllll}2 & 25.3 & 8.1 & 7.7 & 373\end{array}$

$\begin{array}{lllll}3 & 25.3 & 8.1 & 7.7 & 374\end{array}$

$\begin{array}{lllll}4 & 25.0 & 8.0 & 7.7 & 367\end{array}$

$\begin{array}{lllll}5 & 25.3 & 8.4 & 7.6 & 368\end{array}$

$\begin{array}{lllll}6 & 25.4 & 8.4 & 7.6 & 368\end{array}$

$\begin{array}{lllll}\text { MEAN } & 25.1 & 8.2 & 7.7 & 371\end{array}$

MIN $\quad 24.6 \quad 8.0 \quad 7.6 \quad 367$

$\begin{array}{lllll}\operatorname{MaX} & 25.4 & 8.4 & 7.8 & 375\end{array}$

PCM $5.1-100 \%$

INITIAL WARMED

DAY TEMP TEMP DO PH COND HARD ALK CHLORINE

$\begin{array}{lllllllll}0 & 1.5 & 25.1 & 7.6 & 7.3 & 405 & 162.5 & 120 & 10.1\end{array}$

$\begin{array}{llllll}1 & 3.8 & 24.7 & 8.1 & 7.4 & 405\end{array}$

$\begin{array}{lllllllll}2 & 0.5 & 25.2 & 6.9 & 7.6 & 398 & 159.0 & 120 & 10.1\end{array}$

$\begin{array}{llllll}3 & 3.0 & 25.0 & 7.1 & 7.6 & 397\end{array}$

$\begin{array}{llllll}4 & 3.6 & 24.8 & 7.6 & 7.6 & 398\end{array}$

$\begin{array}{llllll}6 & 3.2 & 25.2 & 8.3 & 7.4 & 403\end{array}$

$\begin{array}{lllllllll}\text { MEAN } & 2.3 & 25.0 & 7.6 & 7.5 & 401 & 161.3 & 122 & 10.1\end{array}$

$\begin{array}{lllllllll}\text { MIN } & 0.5 & 24.7 & 6.9 & 7.3 & 397 & 159.0 & 120 & 10.1\end{array}$ $\begin{array}{lllllllll}\operatorname{MAX} & 3.8 & 25.2 & 8.3 & 7.6 & 405 & 162.5 & 127 & 10.1\end{array}$ 
PCY $4.3-50 \%$

INITIAL WARMED

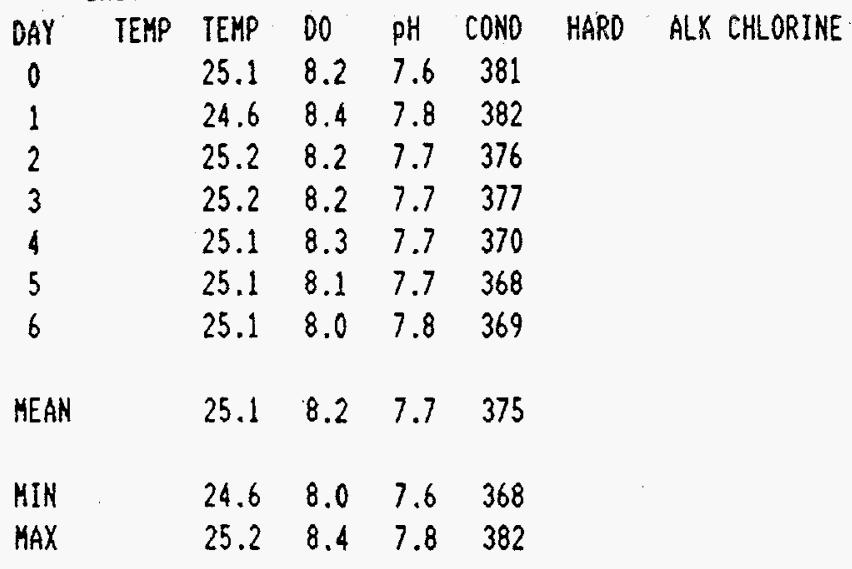

FISH FINAL

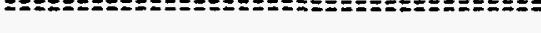

FISH MEDIUH

PCN $2.9-50 \%$

DAY TEMP DO PH COND

$\begin{array}{llll}1 & 24.2 & 7.1 & 7.9\end{array}$

$23.5 \quad 7.1 \quad 363$

$24.0 \quad 6.1$

$23.7 \quad 7.0$

$\begin{array}{lll}2 & 24.7 & 6.8\end{array}$

25.36 .37 .9
-25.26 .9

$\begin{array}{lll}25.2 & 6.9 & 354\end{array}$

25.26 .0

$3 \quad 24.7 \quad 6.8$

$25.1 \quad 6.6$

$\begin{array}{lll}25.0 & 6.7 & 7.9\end{array}$

$\begin{array}{llll}24.9 & 6.7 & 354\end{array}$

$\begin{array}{llll}4 & 25.3 & 6.1 & 356\end{array}$

$\begin{array}{ll}25.2 & 6.2\end{array}$

$25.2 \quad 6.5$

$\begin{array}{lll}25.2 & 6.8 & 7.9\end{array}$

$\begin{array}{llll}5 & 25.5 & 5.5 & 7.7\end{array}$

$25.8 \quad 4.9 \quad 344$

$25.9 \quad 5.0$

$25.8 \quad 4.7$

$6 \quad 25.0 \quad 5.4$

$25.1 \quad 5.0 \quad 7.6$

$25.2 \quad 4.6$

$25.2 \quad 5.4$

$\begin{array}{lll}7 & 24.4 & 5.7\end{array}$

$24.6 \quad 5.6$

$\begin{array}{lll}24.6 & 4.9 \quad 7.6\end{array}$

$24.7 \quad 5.9 \quad 345$

345

56

34

MEAN $24.9 \quad 6.0 \quad 7.8 \quad 352$

$\begin{array}{lllll}\text { MIN } & 23.5 & 4.6 & 7.6 & 344\end{array}$

$\begin{array}{lllll}\text { HAX } & 25.9 & 7.1 & 7.9 & 363\end{array}$
DAY TEMP DO PH COND

$\begin{array}{llll}1 & 24.2 & 6.7 & 8.0\end{array}$

$24.1 \quad 7.0 \quad 371$

$23.9 \quad 6.6$

$24.1 \quad 6.6$

$2 \quad 24.5 \quad 6.5$

$\begin{array}{lll}25.1 & 6.7 & 8.0\end{array}$

$\begin{array}{lll}24.8 & 6.6 \quad 356\end{array}$

$25.0 \quad 6.3$

$3 \quad 25.0 \quad 6.5$

$25.0 \quad 6.2$

$\begin{array}{lll}24.9 & 5.6 & 7.8\end{array}$

$\begin{array}{lll}24.8 & 6.3 & 365\end{array}$

$\begin{array}{llll}425.2 & 6.6 & 366\end{array}$

$25.0 \quad 6.6$

$25.0 \quad 6.5$

$\begin{array}{lll}25.0 & 6.8 & 7.9\end{array}$

$\begin{array}{llll}5 & 25.6 & 4.7 & 7.7\end{array}$

$26.0 \quad 5.5 \quad 360$

$25.8 \quad 5.7$

$25.8 \cdot 5.4$

$6 \quad 25.2 \quad 4.5$

$\begin{array}{lll}25.2 & 4.8 & 7.7\end{array}$

$25.2 \quad 5.3$

$25.1 \quad 5.0$

$7 \quad 24.5 \quad 5.9$

$24.6 \quad 5.7$

$\begin{array}{lll}24.2 & 6.2 & 7.9\end{array}$

$24.8 \quad 5.1 \quad 364$

MEAN $24.9 \quad 6.0 \quad 7.9 \quad 364$

MIN $\quad \begin{array}{lllll}23.9 & 4.5 & 7.7 & 356\end{array}$ $\begin{array}{lllll}\text { MAX } & 26.0 & 7.0 & 8.0 & 371\end{array}$
PCY $2.9-100 \%$

\begin{tabular}{|c|c|c|c|c|}
\hline DAY & TEMP & 00 & $\mathrm{pH}$ & COND \\
\hline \multirow[t]{4}{*}{1} & 23.9 & 6.9 & 8.1 & \\
\hline & 24.0 & 6.5 & & 370 \\
\hline & 24.2 & 6.7 & & \\
\hline & 24.1 & 7.1 & & \\
\hline \multirow[t]{4}{*}{2} & 24.7 & 6.5 & & \\
\hline & 25.2 & 6.6 & 8.0 & \\
\hline & 25.0 & 6.0 & & 370 \\
\hline & 25.1 & 6.9 & & \\
\hline \multirow[t]{4}{*}{3} & 24.6 & 6.7 & & \\
\hline & 25.0 & 7.0 & & \\
\hline & 24.8 & 6.8 & 8.0 & \\
\hline & 24.8 & 6.4 & & 380 \\
\hline \multirow[t]{4}{*}{4} & 25.0 & 6.7 & & 380 \\
\hline & 25.1 & 6.7 & & \\
\hline & 25.1 & 6.7 & & \\
\hline & 25.0 & 6.0 & 7.9 & \\
\hline \multirow[t]{4}{*}{5} & 25.5 & 5.2 & 7.9 & \\
\hline & 25.8 & 4.9 & & 381 \\
\hline & 25.7 & 5.0 & & \\
\hline & 25.9 & 5.0 & & \\
\hline \multirow[t]{4}{*}{6} & 25.2 & 5.1 & & \\
\hline & 25.4 & 5.0 & 7.8 & \\
\hline & 25.0 & 5.0 & & 384 \\
\hline & 25.1 & 4.8 & & \\
\hline \multirow[t]{4}{*}{7} & 24.6 & 5.7 & & \\
\hline & 24.8 & 5.5 & & \\
\hline & 24.8 & 5.8 & 8.0 & \\
\hline & 24.6 & 5.5 & & 383 \\
\hline
\end{tabular}

MEAN $\quad 24.9 \quad 6.0 \quad 8.0 \quad 378$

MIN $\quad 23.9 \quad 4.8 \quad 7.8 \quad 370$

HAX $\quad \begin{array}{lllll}25.9 & 7.1 & 8.1 & 384\end{array}$ 


\begin{tabular}{|c|c|c|c|c|c|c|c|c|c|c|c|c|c|c|}
\hline \multicolumn{5}{|c|}{$P[4.3-508$} & \multicolumn{5}{|c|}{$\operatorname{PCM} 4.3-100 \%$} & \multicolumn{5}{|c|}{ PCM $5.1-50 \%$} \\
\hline DAY & IEKP & DO & $\mathrm{PH}$ & COND & DAY & TEMP & 00 & $\mathrm{PH}$ & CONO & DAY & TEMP & 00 & $\mathrm{pH}$ & COND \\
\hline \multirow[t]{4}{*}{1} & 23.8 & 6.9 & 8.0 & & 1 & 24.3 & 6.0 & 7.8 & & 1 & 24.3 & 7.2 & 8.0 & \\
\hline & 24.1 & 7.0 & & 383 & & 24.3 & 6.9 & & 414 & & 23.7 & 7.2 & & 381 \\
\hline & 24.0 & 6.8 & & & & 24.0 & 7.0 & & & & 23.7 & 6.9 & & \\
\hline & 24.0 & 6.4 & & & & 23.7 & 7.1 & & & & 24.0 & 7.0 & & \\
\hline \multirow[t]{4}{*}{2} & 25.1 & 6.2 & & & 2 & 25.1 & 6.1 & & & 2 & 24.8 & 6.5 & & \\
\hline & 25.4 & 6.4 & 7.9 & & & 25.2 & 6.4 & 7.8 & & & 24.6 & 6.2 & 7.9 & \\
\hline & 24.3 & 6.4 & & 389 & & 25.0 & 5.7 & & 421 & & 24.8 & 6.6 & & 385 \\
\hline & 25.0 & 6.4 & & & & 25.0 & 6.2 & & & & 25.0 & 6.4 & & \\
\hline \multirow[t]{4}{*}{3} & 24.7 & 6.8 & & & 3 & 24.8 & 6.9 & & & 3 & 24.8 & 7.0 & & \\
\hline & 24.7 & 6.3 & & & & 24.9 & 6.9 & & & & 24.8 & 7.1 & & \\
\hline & 24.8 & 6.7 & 8.0 & & & 24.5 & 7.0 & 7.9 & & & 25.0 & 6.7 & 8.0 & \\
\hline & 24.5 & 6.8 & & 379 & & 25.1 & 6.9 & & 405 & & 25.0 & 6.6 & & 375 \\
\hline \multirow[t]{4}{*}{4} & 25.2 & 6.5 & & 379 & 4 & 25.0 & 6.3 & & 406 & 4 & 25.2 & 6.5 & & 377 \\
\hline & 25.3 & 6.3 & & & & 25.1 & 6.5 & & & & 24.5 & 6.6 & & \\
\hline & 24.4 & 6.8 & & & & 24.8 & 6.5 & & & & 24.7 & 6.7 & & \\
\hline & 25.0 & 6.3 & 7.9 & & & 24.9 & 6.5 & 8.0 & & & 24.9 & 6.2 & 7.9 & \\
\hline \multirow[t]{4}{*}{5} & 25.6 & 5.6 & 7.8 & & 5 & 25.9 & 5.8 & 7.9 & & 5 & 25.7 & 5.8 & 7.8 & \\
\hline & 25.4 & 5.8 & & 371 & & 25.9 & 5.0 & & 409 & & 25.7 & 5.7 & & 371 \\
\hline & 25.5 & 5.8 & & & & 25.8 & 5.3 & & & & 25.6 & 5.9 & & \\
\hline & 25.8 & 5.4 & & & & 25.9 & 5.0 & & & & 25.8 & 4.6 & & \\
\hline \multirow[t]{4}{*}{6} & 24.9 & 4.3 & & & 6 & 25.2 & 4.8 & & & 6 & 24.7 & 5.0 & & \\
\hline & 25.0 & 4.8 & 7.7 & & & 25.1 & 4.5 & 7.8 & & & 25.0 & 5.0 & 7.7 & \\
\hline & 24.7 & 5.4 & & 375 & & 25.2 & 4.8 & & 409 & & 25.1 & 5.7 & & 380 \\
\hline & 25.4 & 4.9 & & & & 25.2 & 4.6 & & & & 25.2 & 4.1 & & \\
\hline \multirow[t]{4}{*}{7} & 24.8 & 5.4 & & & 7 & 24.8 & 4.6 & & & 7 & 24.8 & 5.7 & & \\
\hline & 24.8 & 4.6 & & & & 24.3 & 5.2 & & & & 24.6 & 5.3 & & \\
\hline & 24.7 & 4.8 & 7.7 & & & 24.8 & 5.3 & 7.9 & & & 23.7 & 6.2 & 7.9 & \\
\hline & 24.6 & 5.2 & & 371 & & 24.7 & 5.0 & & 405 & & 24.5 & 4.6 & & 377 \\
\hline MEAN & 24.8 & 6.0 & 7.9 & 378 & MEAN & 24.9 & 5.9 & 7.9 & 410 & MEAH & 24.8 & 6.1 & 7.9 & 378 \\
\hline MIN & 23.8 & 4.3 & 7.7 & 371 & MIN & 23.7 & 4.5 & 7.8 & 405 & MIN & 23.7 & 4.1 & 7.7 & 371 \\
\hline MAX & 25.8 & 7.0 & 8.0 & 389 & MAX & 25.9 & 7.1 & 8.0 & 421 & MAX & 25.8 & 7.2 & 8.0 & 385 \\
\hline
\end{tabular}


PCH $5.1-1003$

\begin{tabular}{|c|c|c|c|c|c|}
\hline DAY & TEMP & 00 & $\mathrm{PH}$ & COND & $\because \ldots \quad \cdots$ \\
\hline \multirow[t]{4}{*}{1} & 24.1 & 6.7 & 8.0 & & \\
\hline & 23.8 & 6.8 & & 408 & \\
\hline & 24.0 & 7.2 & & & MEAN TEMPERATURE 24.9 \\
\hline & 23.5 & 7.2 & & & \\
\hline \multirow[t]{4}{*}{2} & 25.0 & 6.6 & & & MINIMUK: 23.5 \\
\hline & 25.0 & 6.4 & 7.9 & & MAXIMUM: 26.0 \\
\hline & 25.2 & 6.2 & & 408 & \\
\hline & 24.5 & 6.2 & & & \\
\hline \multirow[t]{4}{*}{3} & 24.8 & 6.6 & & & \\
\hline & 25.0 & 6.5 & & & \\
\hline & 24.7 & 6.5 & & & \\
\hline & 24.9 & 6.5 & 8.0 & 403 & \\
\hline \multirow[t]{4}{*}{4} & 24.8 & 6.5 & & 401 & \\
\hline & 24.9 & 6.8 & & & \\
\hline & 25.3 & 6.7 & & & \\
\hline & 25.0 & 6.2 & 7.9 & & \\
\hline \multirow[t]{4}{*}{5} & 25.9 & 5.5 & 7.9 & & \\
\hline & 25.7 & 5.4 & & 401 & \\
\hline & 25.6 & 5.6 & & & \\
\hline & 25.6 & 5.3 & & & \\
\hline \multirow[t]{4}{*}{6} & 25.0 & 4.7 & & & \\
\hline & 25.4 & 4.8 & 7.8 & & \\
\hline & 25.2 & 4.9 & & 410 & \\
\hline & 25.2 & 4.8 & & & \\
\hline \multirow[t]{4}{*}{7} & 24.4 & 5.6 & & & \\
\hline & 24.4 & 5.8 & & & \\
\hline & 24.9 & 5.4 & 7.8 & & \\
\hline & 24.8 & 5.7 & & 407 & \\
\hline MEAN & 24.9 & 6.0 & 7.9 & 405 & \\
\hline $\operatorname{MIN}$ & 23.5 & 4.7 & 7.8 & 401 & \\
\hline MAK & 25.9 & 7.2 & 8.0 & 410 & \\
\hline
\end{tabular}


INITIAL CHEMISTRY

study CR-ERP OAt 93

Beginning Date $10-21.93$

Date $10-21$
Page 1 of 7

Personnel Simbeck

Pose y

Ending Date $10-28-93$

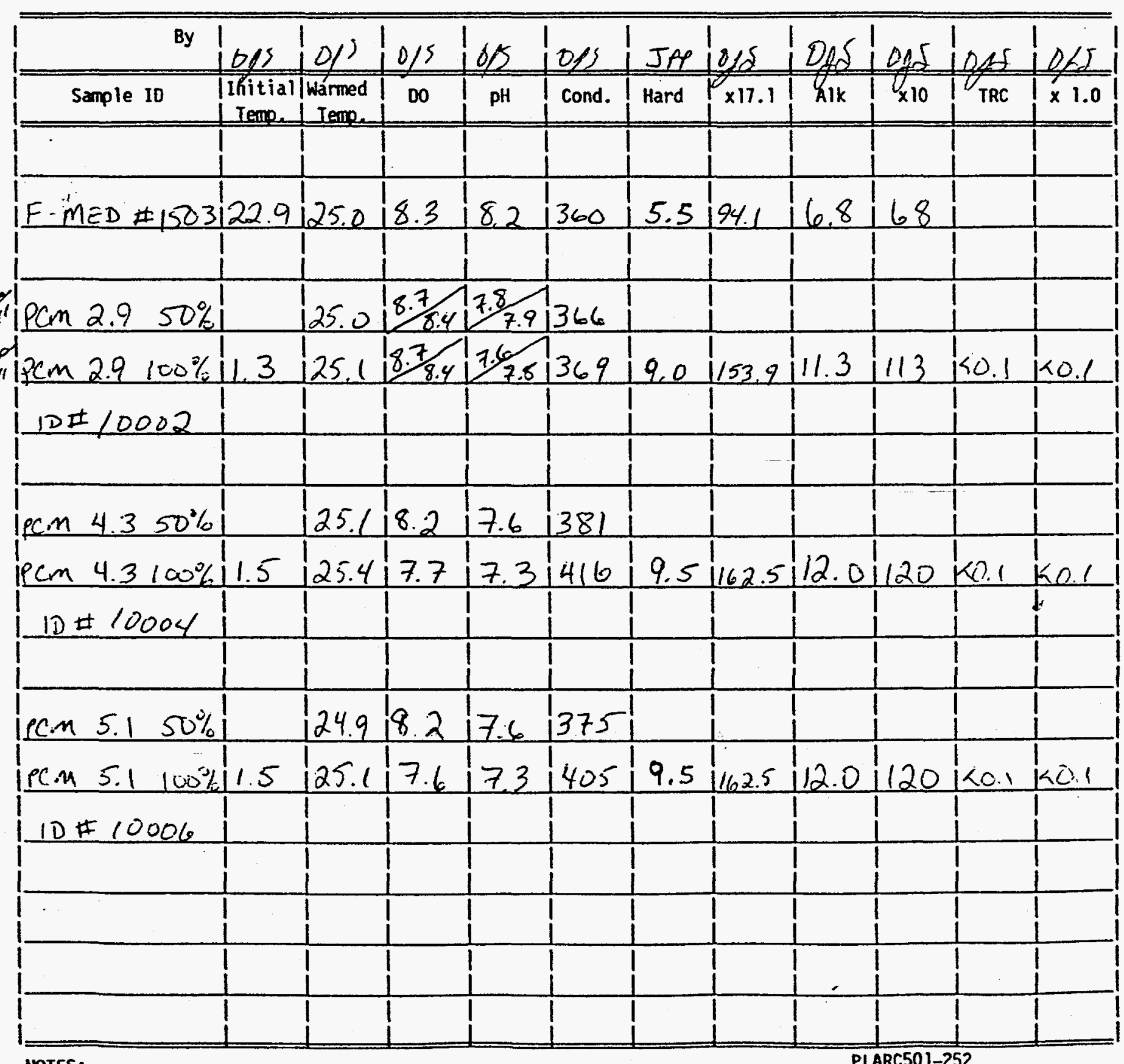

NOTES:

PLARC501-252

Reviewed By: 
INITIAL CHEMISTRY

Page 2 of 7

study CR-ERP Oat 93

Date $10-22$

Personnel Simbeck

Beginning Date $10 \cdot 21.93$

Posey

Ending Date $10-28-93$

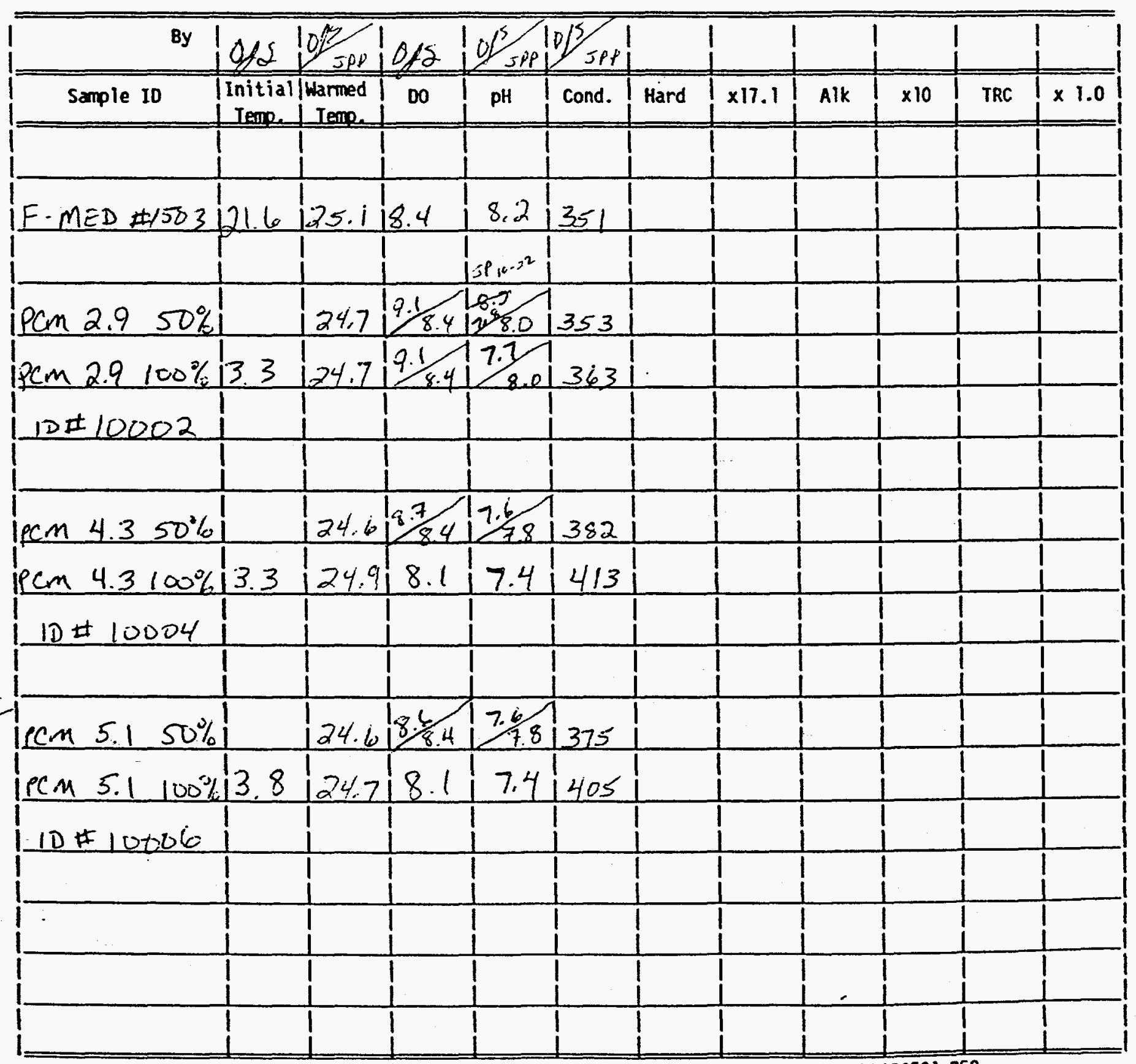

MOTES:

PLARC501-252 
INITIAL CHEMISTRY

Page 3 of 7

study $C R$-ERP Oct 93

Date $10-23-93$

Personnel Simbeck

Beginning Date $10-21.93$

Posey

Ending Date $10-28-93$

\begin{tabular}{|c|c|c|c|c|c|c|c|c|c|c|c|}
\hline By 1 & 1045 & $1005 / 5$ spe & d1/ $/ 2 p$ & 15 & $0+2 / 5 p p$ & JPP & 0,2 & JPP & 0,5 & JPP & $\tan 2$ \\
\hline Sample 10 & $\begin{array}{l}\text { Ifitial } \\
\text { Iemp. }\end{array}$ & $\begin{array}{l}\text { I Warmed } \\
\text { Lemp. }\end{array}$ & 00 & pH & cond. & Hard & $\times 17.1$ & Alk & $\times 10$ & TRC & $\times 1.0$ \\
\hline & & & & & & & & & & & \\
\hline F-MED \#1503 & 122.2 & $|25,2|$ & 8.4 & 8.2 & 351 & & & & & & \\
\hline & & & & & & & & & & & \\
\hline $\operatorname{Pcm} 2.950 \%$ & & 25,3 & $\frac{8.7 / 8}{8.2}$ & $\frac{7.9 / 8}{8.0}$ & 364 & & & & & & \\
\hline $\operatorname{Pcm} 2.9 \quad 100 \% !$ & 10.5 & 25.31 & 8.0 & 7.7 & 378 & 9.0 & 1539 & 11.7 & 1117 & $<0,1$ & $<0.1$ \\
\hline DIt1003Q & & & & & & & & & & & \\
\hline & & & & & & & & & & & \\
\hline ecm $4.350 \%$ & & 25.21 & 8.2 & 7.7 & 376 & & & & & & \\
\hline $\operatorname{lcm} 4.3100 \%$ & 0.4 & 25.11 & 7.0 & 7,5 & 4031 & 9.3 & 11590 & 12.0 & 120 & $<0.1$ & $<0.1$ \\
\hline $1 D+10034$ & & & & & & & & & & & \\
\hline & & & & & & & & & & & \\
\hline $\operatorname{sem} 5.150 \%$ & & 25.31 & 8.1 & 7.7 & 373 & & & & & & \\
\hline$p C M \quad 5.1 \quad 100 \%$ & 0.5 & 25.21 & 6.9 & 7.6 & 398 & 9,3 & 11590 & 12.01 & 1120 & $<0 . i$ & $<0.1$ \\
\hline $10 \# 10036$ & & & & & & & & & & & \\
\hline & & & & & & & & & & & \\
\hline & & & & & & & & & & & \\
\hline & & & & & & & & & & & \\
\hline & & & & & & & & & & & \\
\hline
\end{tabular}

NOTES:

PLARC501-252

Reviewed By: 25 
INITIAL CHEMISTRY

Page 4 of 7

study CR-ERP Oct' 93

Date $10-24$

Personnel Simback

Beginning Date $10-21.93$

Posey

Ending Date $10-28-93$

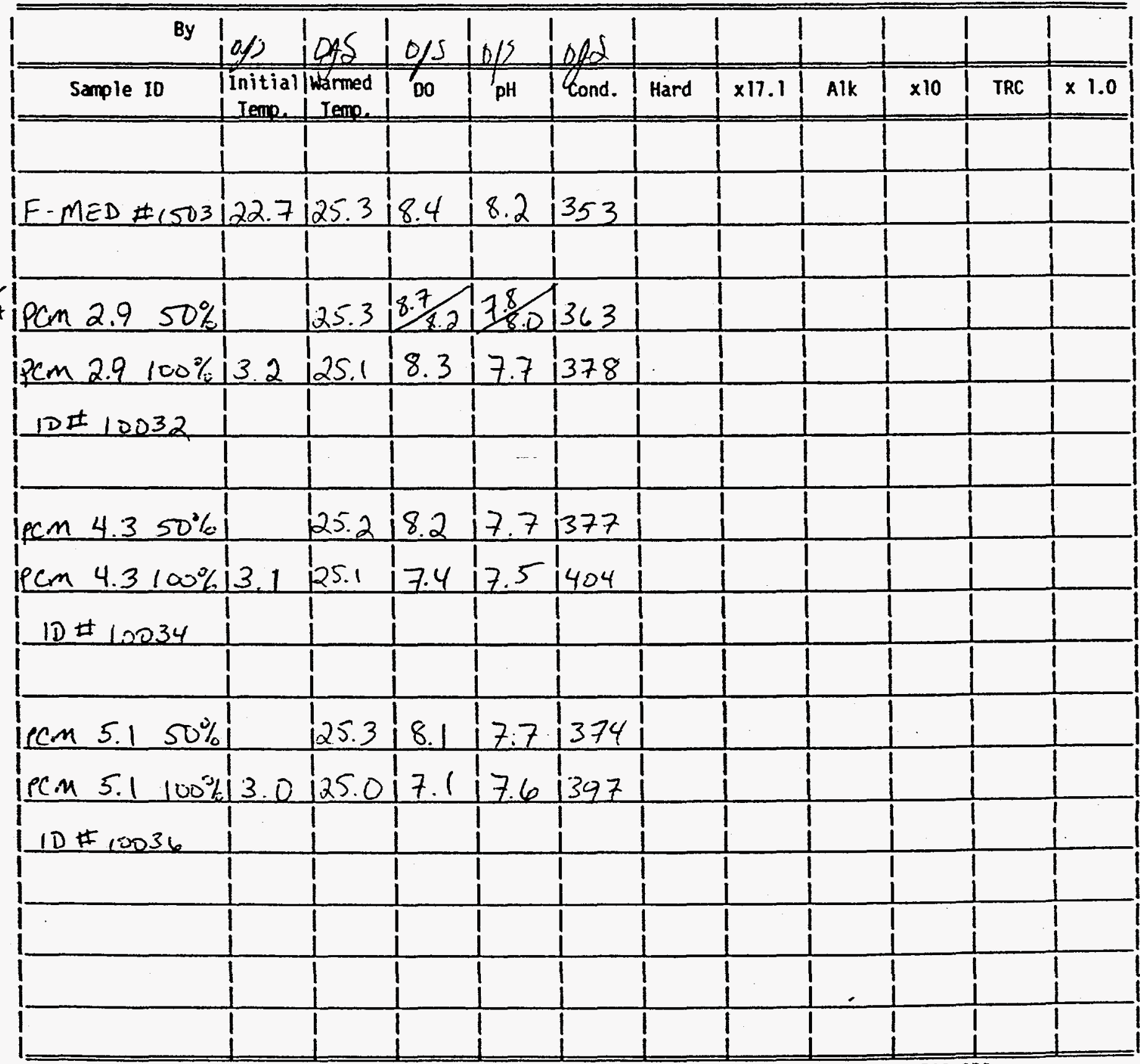

MOTES:

PLARC501-252 
INITIAL CHEMISTRY

Page 5 of 7

Study C'R-ERP Oct 93

Date $10-25$

Personnel Simback

Beginning Date $10-21.93$

Posey

Ending Date $10-28-93$

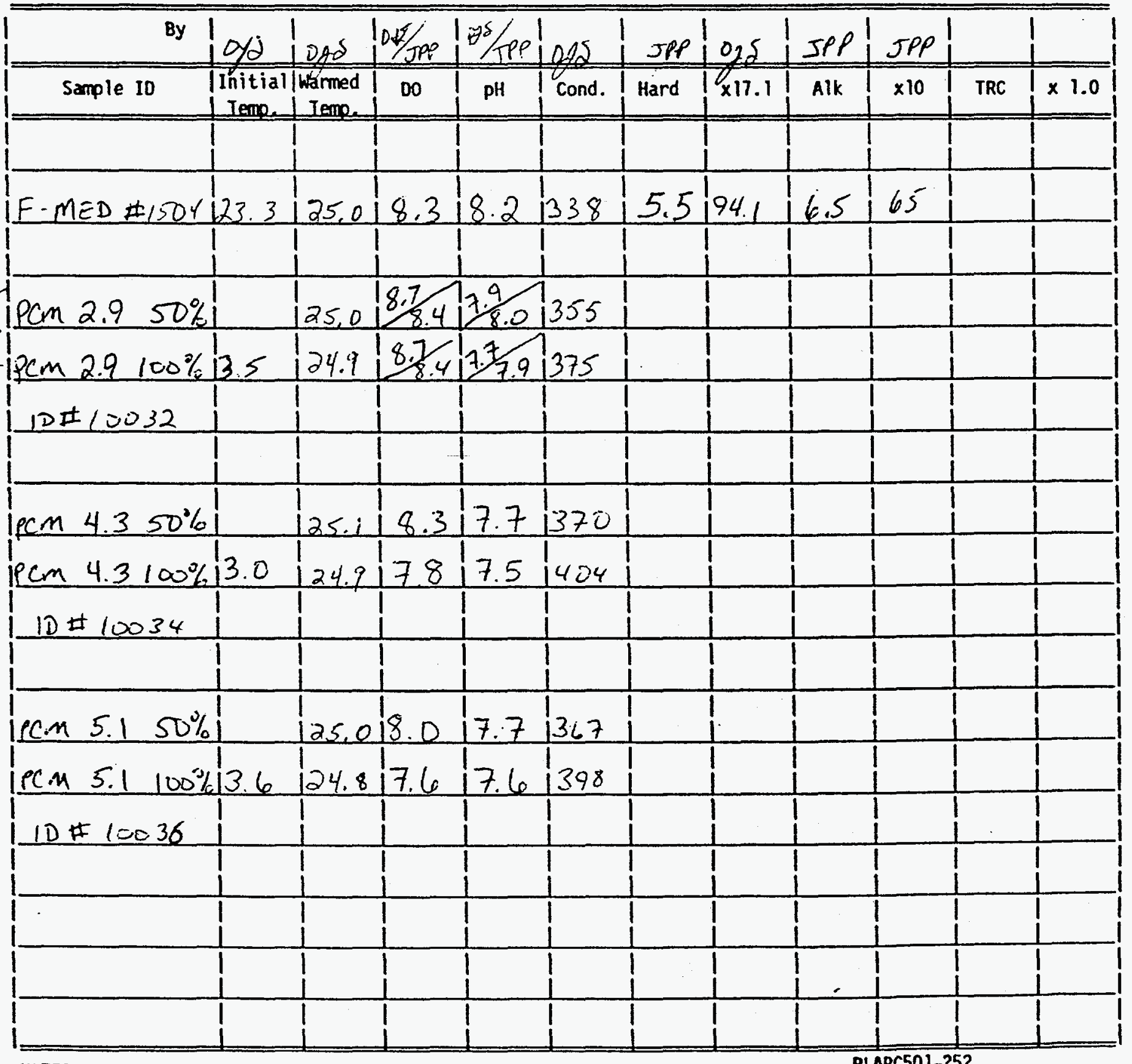

NOTES:

PLARC501-252 
INITIAL CHEMISTRY

study $C R-E R P$ Oct 93

Beginning Date 10.21 .93

Date $10-26$
Page 6 of 7

Personne1 Simback

Posey

Ending Date $10-28-93$

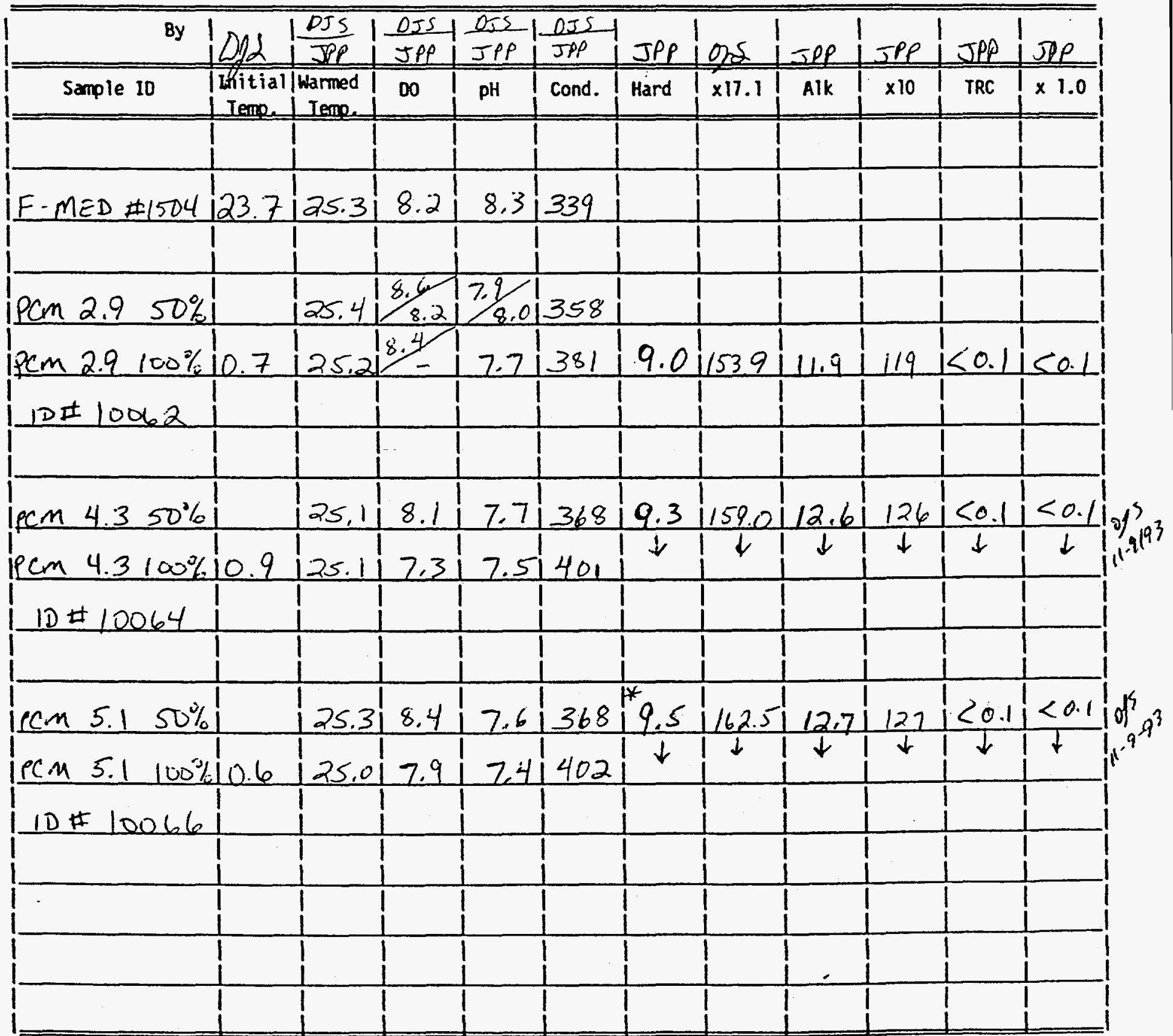

NOTES:

* checked tarie 
INITIAL CHEMISTRY

Page 7 of 7

study CR-ERP Oct'93

Beginning Date $10-21.93$

Date $10-27-93$
Personnel Simback

Posey

Russeel

$\frac{0855}{0.58}$

Ending Date $10-28-93$

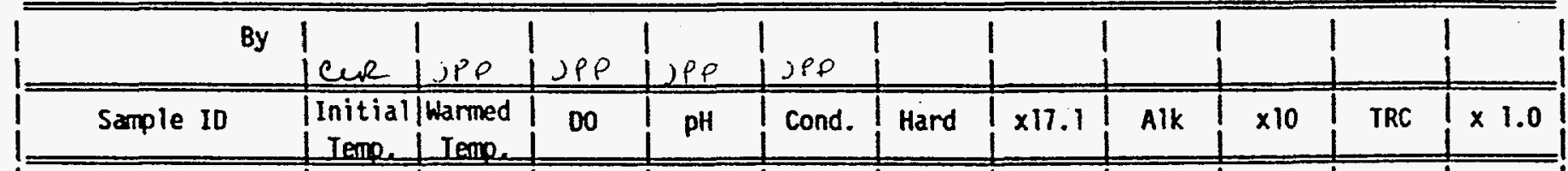

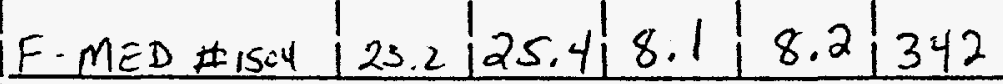

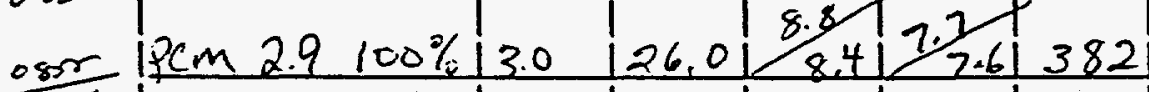

0858

IDI 10062

\begin{tabular}{l|l|l|l|l|}
\hline lecm $4.350 \%$ & 25.1 & 8.0 & 7.8 & 369
\end{tabular}

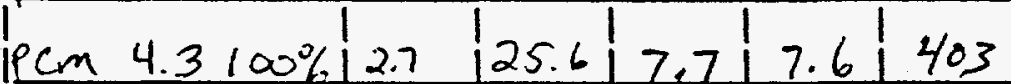

ID $\# 10064$

\begin{tabular}{|ll|l|l|l|l|}
\hline Iec.n $5.150 \%$ & 25.4 & 8.4 & 7.6 & 368 \\
\hline
\end{tabular}

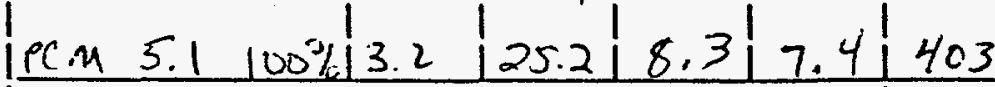

$10 \# 10064$

\begin{tabular}{|l|l|}
\hline & \\
\hline & 1 \\
\hline & 1 \\
\hline & 1 \\
\hline & 1 \\
\hline
\end{tabular}

MOTES:

PLARC501-252 
FINAL CHEMISTRY

Study CR-ERP OCt 193

Beginning Date $10-21-93$

Ending Date $10-28-43$

\begin{tabular}{|c|c|c|c|c|c|}
\hline $\begin{array}{l}\text { Sample } 10 \\
\text { S= }\end{array}$ & $\begin{array}{c}====== \\
\text { By } \\
==== \pm= \\
\text { Rep. } \\
== \pm== \pm=\end{array}$ & 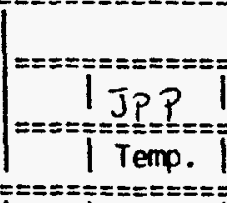 & $\begin{array}{l}\text { Fish } \\
===== \pm= \\
\text { JPP } \\
\text { DP }=== \\
======\end{array}$ & 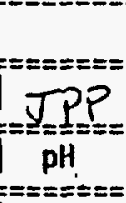 & $\begin{array}{l}======= \\
\mid \text { JPP } \\
\mid===== \\
\text { Cond. }\end{array}$ \\
\hline & 1 & 24.2 & 7.1 & 7.9 & \\
\hline$E-M \in D$ & 2 & 23.5 & 7.1 & & 363 \\
\hline Control & 3 & 24.0 & 6.1 & & \\
\hline $10 \pm 091,00.43$ & 4 & 23.7 & 7.0 & & \\
\hline & 1 & 24.3 & 6.7 & 8.0 & \\
\hline PCM 2.9 & 2 & 24.1 & 7.0 & & $37 \mid$ \\
\hline $50 \%$ & 3 & 239 & 6.6 & & \\
\hline & 4 & 424.1 & 6.6 & & \\
\hline & 1 & 23,9 & 6.9 & 8.1 & \\
\hline PCM 2.9 & 2 & 24.0 & 6.5 & & 370 \\
\hline $100 \%$ & 3 & 24.2 & 6.7 & & \\
\hline & 4 & 24.1 & 7.1 & & \\
\hline & 1 & 23.8 & 6.9 & 8.0 & \\
\hline $\operatorname{pcm} 4.3$ & 2 & 24.1 & 70 & & 383 \\
\hline $30 \%$ & 3 & 24.0 & 10.8 & & \\
\hline & 4 & 240 & 6.4 & & \\
\hline & 1 & 24.3 & 6.0 & 78 & \\
\hline PCn 4.3 & 2 & 24.3 & 6.9 & & 414 \\
\hline $100 \%$ & 3 & 24.0 & 7.0 & & \\
\hline & 4 & 237 & 7.1 & & \\
\hline & 1 & 24.3 & 7.2 & 8.0 & \\
\hline$P C \cdot n 5.1$ & 2 & 237 & 7.2 & & 381 \\
\hline $50 \%$ & 3 & 23.7 & 6.9 & & \\
\hline & 4 & 24.0 & 7.0 & & \\
\hline & 1 & 24.1 & 6.7 & 8.0 & \\
\hline PCM 5.1 & 2 & 23.8 & 6.8 & & 408 \\
\hline $150 \%$ & 3 & 24.0 & 7.2 & & \\
\hline & 4 & 23.5 & 7.2 & & \\
\hline
\end{tabular}

NOTES:
Page 1 of 7

Personnel Simbeck Posey

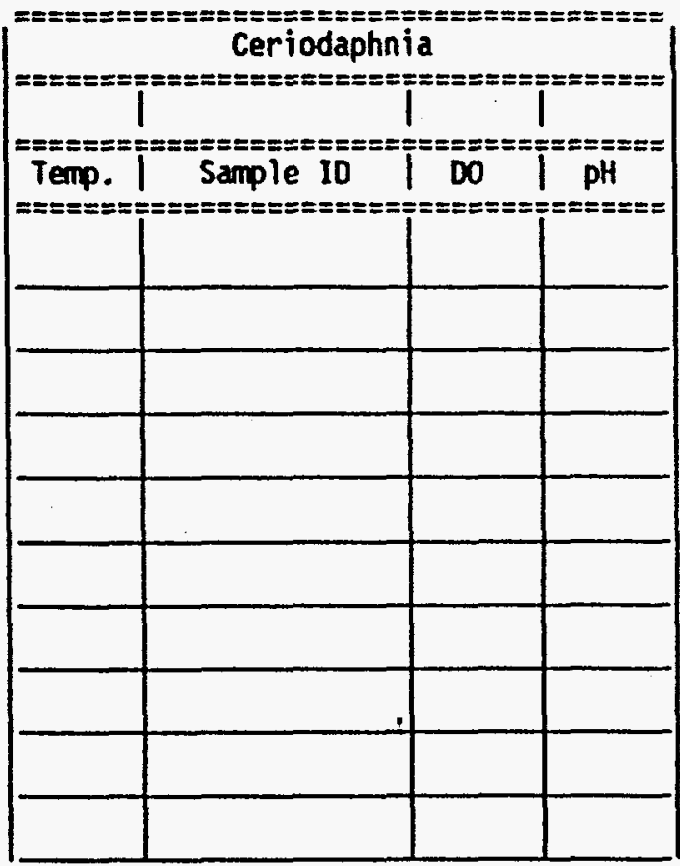

Reviewed By: 25 
FINAL. CHEMISTRY

Study $S R-E R P \quad Q<1 \quad 93$

Beginning Date $10.21-93 \quad$ Date $10-23$

Ending Date $10-25-43$

\begin{tabular}{|c|c|c|c|c|c|}
\hline Sample 10 & $\begin{array}{l}=== \pm== \\
=== \pm== \\
\text { By } \\
==== \pm= \\
\text { Rep. } \\
== \pm== \pm=\end{array}$ & 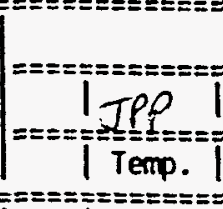 & $\begin{array}{c}\text { Fish } \\
====== \\
J P P_{0}== \\
D 0\end{array}$ & 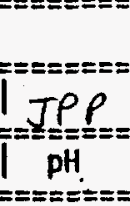 & 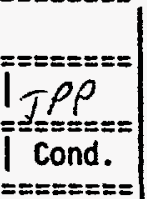 \\
\hline & 1 & 247 & 6.8 & & \\
\hline$E-M \in D$ & 2 & 253 & 6.3 & 7.9 & \\
\hline Contral & 3 & 25.2 & 69 & & 354 \\
\hline 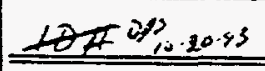 & 4 & 25,2 & $\ln .0$ & & \\
\hline & 1 & 24.5 & $\ln .5$ & & \\
\hline PCM 2.9 & 2 & 25.1 & 6.7 & 8.0 & \\
\hline $50 \%$ & 3 & 24.8 & 6.6 & & 20351 \\
\hline & 4 & 25.0 & 6.3 & & \\
\hline & $T$ & 247 & 6.5 & & \\
\hline $\operatorname{PCn} 2.9$ & 2 & 25.2 & 6.6 & 8.0 & \\
\hline $100 \%$ & 3 & 25.0 & 60 & & 370 \\
\hline & 4 & 25.1 & 6.9 & & \\
\hline & 1 & 25.1 & 6.2 & & \\
\hline $\operatorname{Pcm} 4.3$ & 2 & 25.4 & 10.4 & 7.9 & \\
\hline $50 \%$ & 3 & 24.3 & 16.4 & & 389 \\
\hline & 4 & 25.0 & 6.4 & & \\
\hline & 1 & 25.1 & 6.1 & & \\
\hline $\operatorname{PCn} 4.3$ & 2 & 252 & $\log 4$ & 7.8 & \\
\hline $100 \%$ & 3 & 25.0 & 5.7 & & 421 \\
\hline & 4 & 05.0 & 6.2 & & \\
\hline & 1 & 248 & ia 5 & & \\
\hline PC.M S.1 & 2 & 246 & 10.2 & 7.9 & \\
\hline $20 \%$ & 3 & 24.8 & leda & & 385 \\
\hline & 4 & 25.0 & 6.4 & & \\
\hline & 1 & 25.0 & 1. & & \\
\hline PCM 5.1 & 2 & 25.0 & 164 & 7.9 & $20,5 \cdot 2 \cdot 2$ \\
\hline $1500 \%$ & 3 & 25.2 & 6.2 & & 3408 \\
\hline & 4 & 24.5 & 6.2 & & \\
\hline
\end{tabular}

NOTES:
Page 2 of 7

Personnel Sinbeck Posey

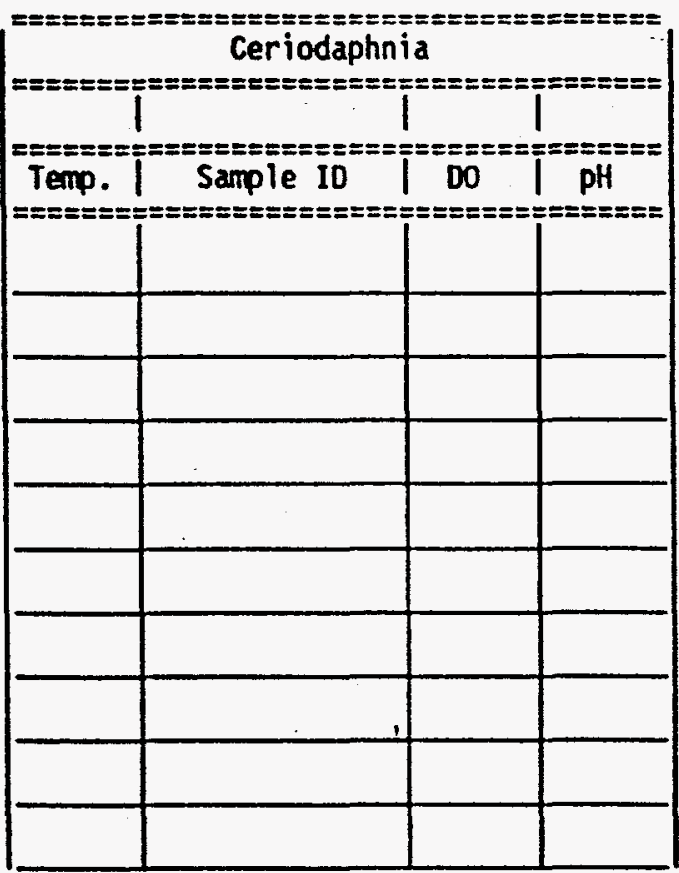

Reviewed By: 25 
FINAL CHEMISTRY

Study SR-ERP OCt 193

Beginning Date $10-21-93 \quad$ Date $10-24$

Ending Date $10-28-43$

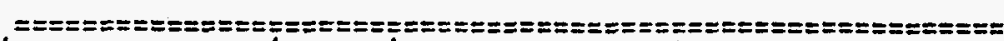

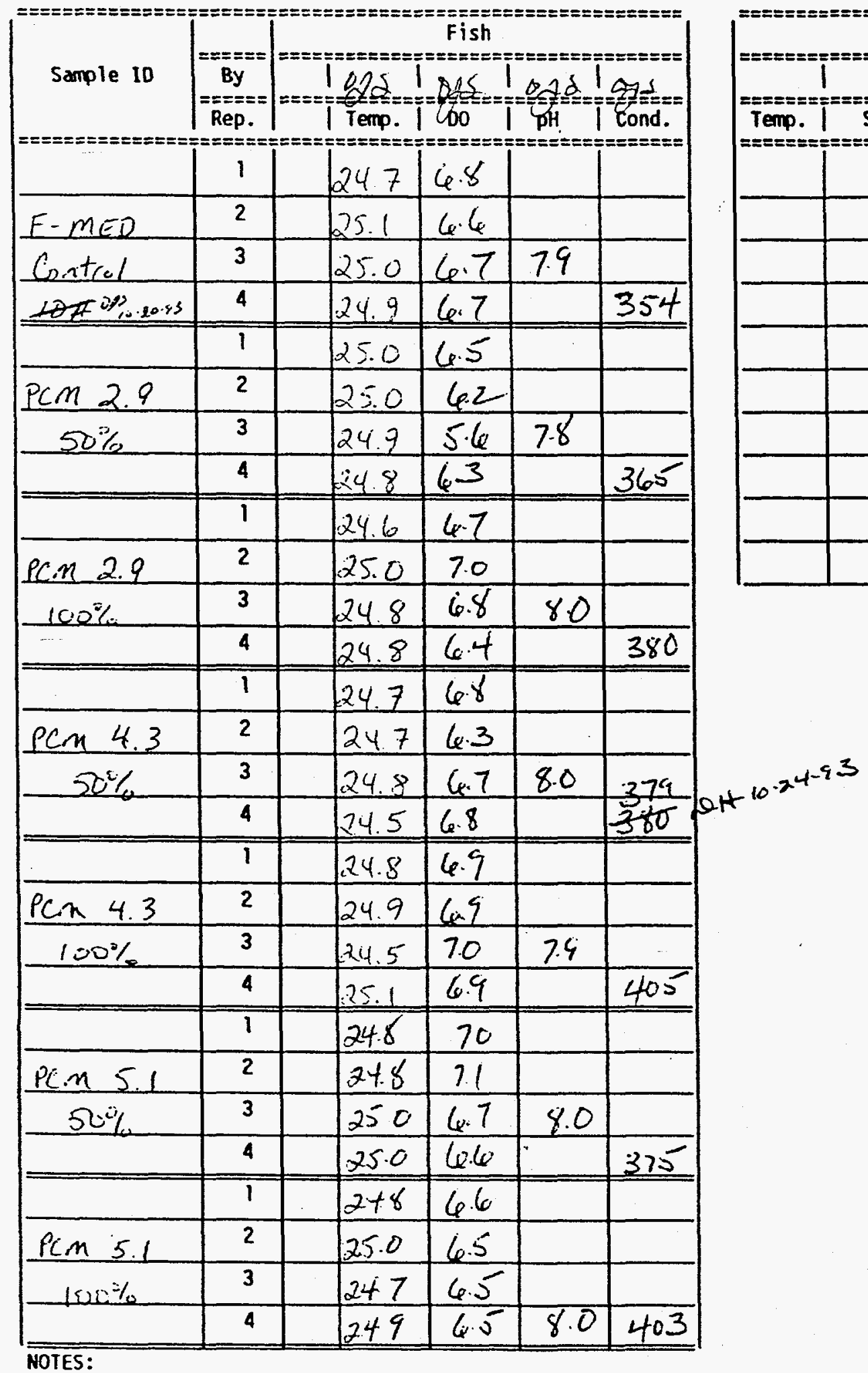

Reviewed By:
Page 3 of 7

Personnel Simbeck

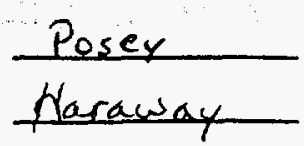

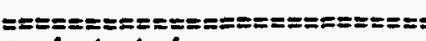
Ceriodaphnia

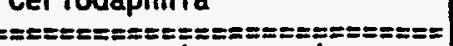

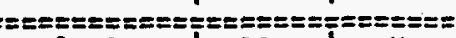

Sample Io I DO I PH

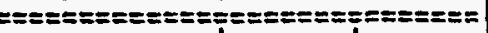

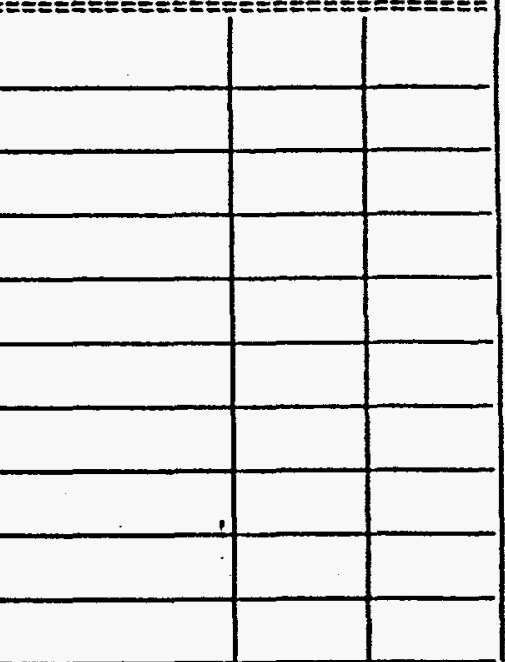


FINAL CHEMISTRY

Study $C R-E R P$ OCt 93

Beginning Date 10.21 .93

Date $10-25$
Page 4 of 7

Personnel Simbeck Posey

Ending Date $10-25.43$

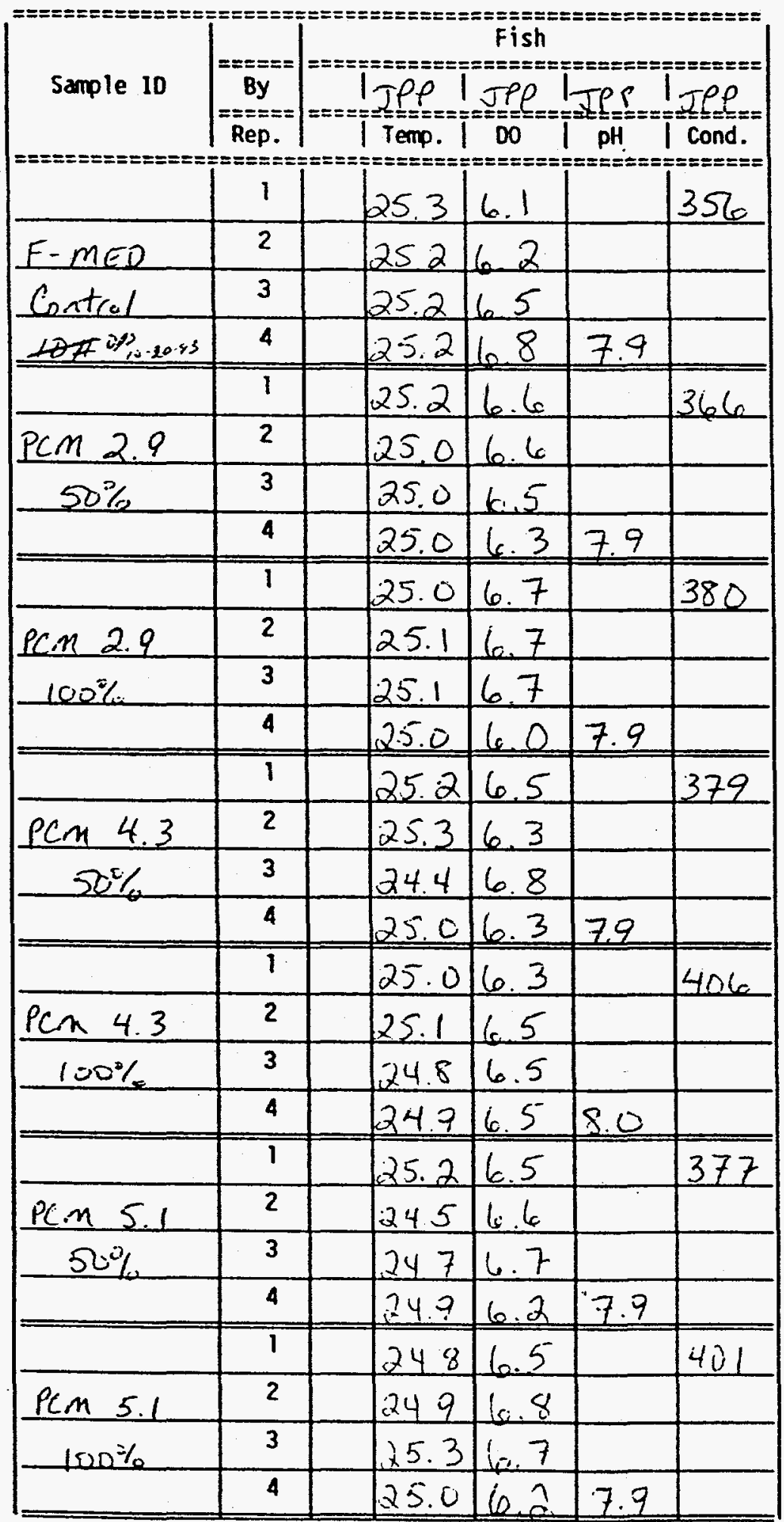

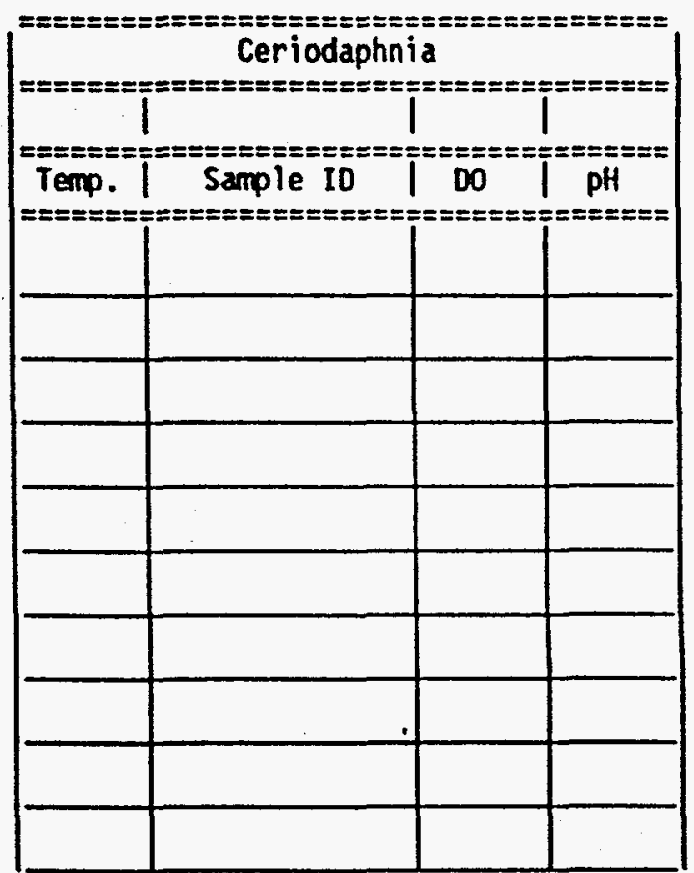

NOTES:

Reviewed By:

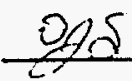


FINAL CHEMISTRY

Study CR-ERP OCt 93

Beginning Date $10-21-93$

Date $10-26$
Page 5 of 7

Personnel Simbeck Poser

Ending Date $10.28-93$

\begin{tabular}{|c|c|c|c|c|c|}
\hline 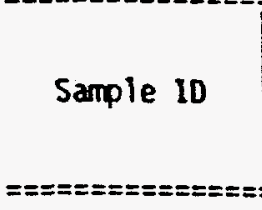 & $\begin{array}{c}====== \\
\text { By } \\
===== \\
\text { Rep. } \\
======\end{array}$ & 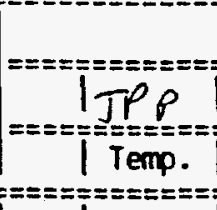 & 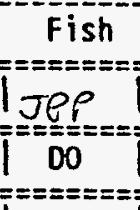 & 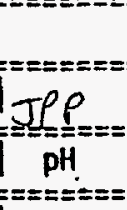 & 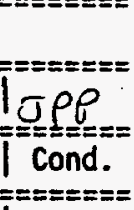 \\
\hline & 1 & 25.5 & 5.5 & 7.7 & \\
\hline$F-M E D$ & 2 & 25.8 & 4.9 & & 344 \\
\hline Contrel & 3 & 25.9 & 5.0 & & \\
\hline 10स $299,20.43$ & 4 & 25.8 & 4.7 & & \\
\hline & 1 & 25.6 & 4.7 & 7.7 & \\
\hline $\operatorname{PCM} 2.9$ & 2 & 26.0 & 5.5 & & 360 \\
\hline $50 \%$ & 3 & 25.8 & 5.87 & 26 & \\
\hline & 4 & 25.8 & 5.4 & & \\
\hline & 1 & 25.5 & 5.2 & 7.9 & \\
\hline PC. 2.9 & 2 & 25.8 & 49 & & 381 \\
\hline $100 \%$ & 3 & 25.7 & 5.0 & & \\
\hline & 4 & 25.9 & 5.0 & & \\
\hline & 1 & 25.6 & 5.6 & 7.8 & \\
\hline Pcm 4.3 & 2 & 25.4 & 5.8 & & 371 \\
\hline $20 \%$ & 3 & 25.5 & 5.8 & & \\
\hline & 4 & 25.8 & 5.4 & & \\
\hline & 1 & 25.9 & 5.8 & 7.9 & \\
\hline $\operatorname{PCn} 4.3$ & 2 & 25.9 & 5.0 & & 409 \\
\hline $100 \%$ & 3 & 25.8 & 5.3 & & \\
\hline & 4 & 25.9 & 5.0 & & \\
\hline & 1 & 25.7 & 5.8 & 7.8 & \\
\hline PC.M 5.1 & 2 & 257 & 5.7 & & 371 \\
\hline $50 \%$ & 3 & 256 & 5.9 & & \\
\hline & 4 & 25.8 & 46 & & \\
\hline & 1 & 25.9 & 55 & 79 & \\
\hline PCM 5.1 & 2 & 25.7 & 5.4 & & 401 \\
\hline $1500 \%$ & 3 & 25.6 & 5.6 & & \\
\hline & 4 & 25.6 & 5.3 & & \\
\hline
\end{tabular}

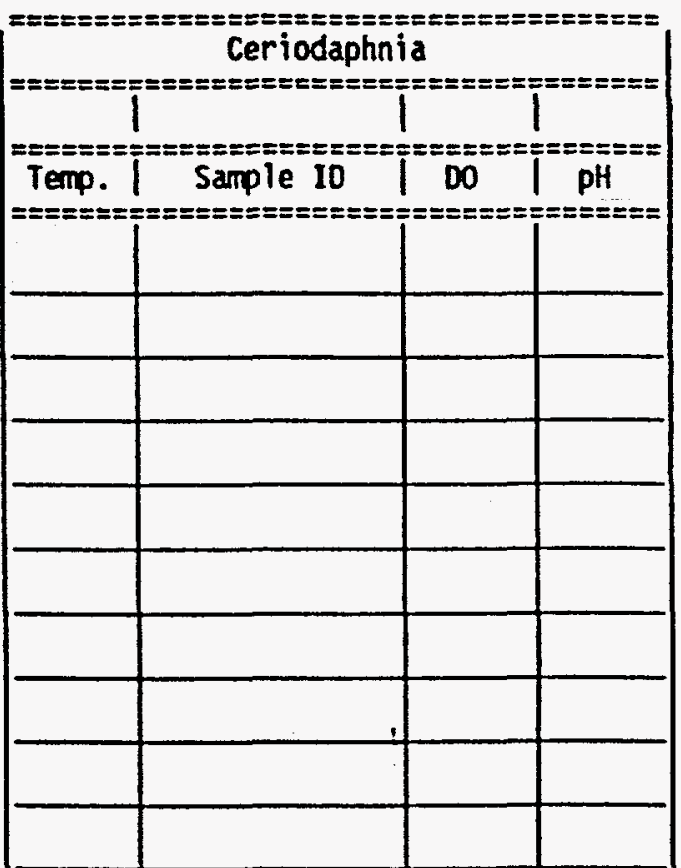

NOTES:

Reviewed By:

92 
FINAL CHEMISTRY

Study $S R-E R P \quad 0<1 \quad 193$

Beginning Date $10-21-23$

Date $10-27$

Ending Date $10-25.43$

\begin{tabular}{|c|c|c|c|c|c|}
\hline \multirow{2}{*}{ 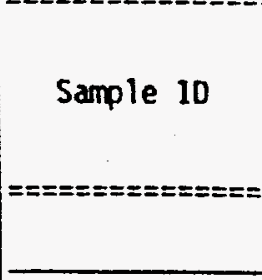 } & $\begin{array}{l}====== \\
\text { By } \\
====== \\
\text { Rep. } \\
z=====\end{array}$ & 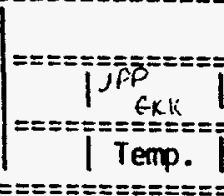 & $\begin{array}{c}\text { Fish } \\
==0.9 K K \\
=0\end{array}$ & $\begin{array}{c}\overline{P P F}==== \\
\mathrm{GKKK} \\
\mathrm{PH}\end{array}$ & 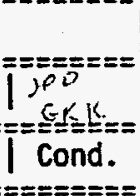 \\
\hline & 1 & 25,0 & 5.4 & & \\
\hline$E-m \in D$ & 2 & 25.1 & 5.0 & 2.6 & \\
\hline Contral & 3 & 25.2 & 46 & & 345 \\
\hline \multirow[t]{2}{*}{$18 \mp 0.00 .20 .45$} & 4 & 25.2 & 5.4 & & \\
\hline & 1 & 25.2 & 4,5 & & \\
\hline PCM 2.9 & 2 & 25.2 & 48 & 7.7 & \\
\hline \multirow[t]{3}{*}{$50 \%$} & 3 & 25,2 & 5,3 & & 365 \\
\hline & 4 & 25.1 & 5.0 & & \\
\hline & 1 & 25.2 & 5.1 & & \\
\hline PC.n 2.9 & 2 & 25.4 & 5.0 & 7.8 & \\
\hline \multirow[t]{3}{*}{$100 \%$} & 3 & 25.0 & 5.0 & & 384 \\
\hline & 4 & 25.1 & 48 & & \\
\hline & 1 & 24.9 & 4.3 & & \\
\hline $\operatorname{Pcm} 4.3$ & 2 & 25.0 & 4.8 & 7.7 & \\
\hline \multirow[t]{3}{*}{$20 \%$} & 3 & 24.7 & 5.4 & & 325 \\
\hline & 4 & 25.4 & 4.9 & & \\
\hline & 1 & 25.2 & 4.8 & & \\
\hline PCn 4.3 & 2 & 25.1 & 4.5 & 7,8 & \\
\hline \multirow[t]{3}{*}{$100 \%$} & 3 & 25.2 & 4.8 & & 409 \\
\hline & 4 & 25.2 & 4.6 & & \\
\hline & 1 & 247 & 5.0 & & \\
\hline$P C=5.1$ & 2 & 25.0 & 5.0 & 2.7 & \\
\hline \multirow[t]{3}{*}{$50 \%$} & 3 & 25.1 & 5.7 & & 380 \\
\hline & 4 & 25.2 & 4.1 & & \\
\hline & 1 & 25.0 & 4.7 & & \\
\hline PCM 5.1 & 2 & 25.4 & 4.8 & 78 & \\
\hline \multirow[t]{2}{*}{$100 \%$} & 3 & 25.2 & 4.9 & & 410 \\
\hline & 4 & 25.2 & 4.8 & & \\
\hline
\end{tabular}

Page 6 of 7

Personnel Simbeck Posey Kincad

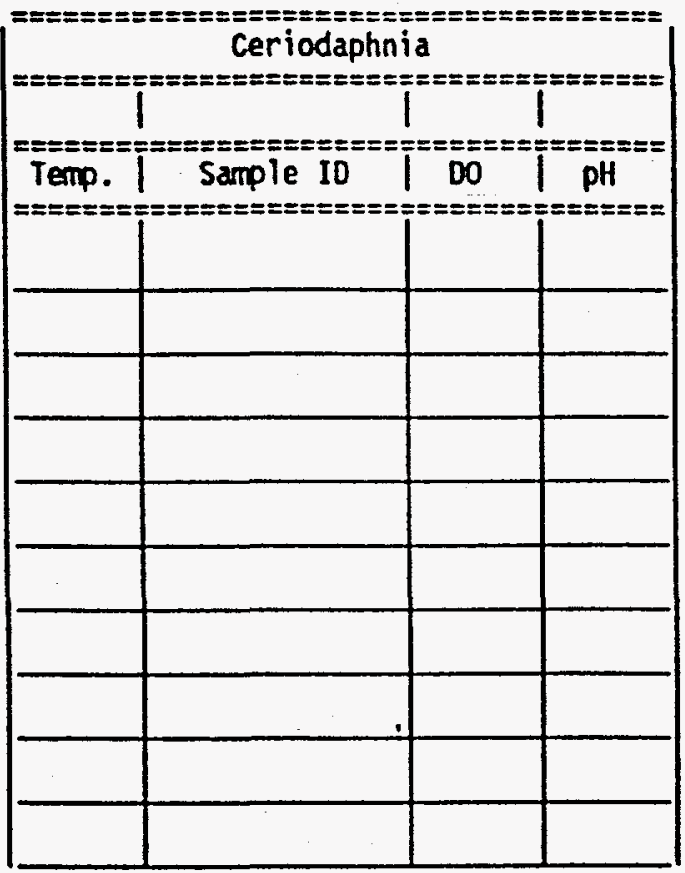

NOTES:

Reviewed 8y: of 
$\because \cdots+$

Study $S R-E R P$ OCt 93

Beginning Date $10-21.93$

FINAL CHEMISTRY

Ending Date $10-28-43$

\begin{tabular}{|c|c|c|c|c|c|}
\hline \multirow{4}{*}{ 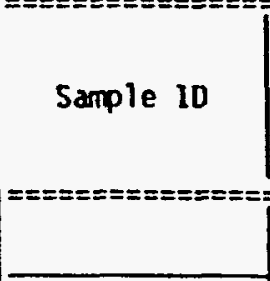 } & \multirow{3}{*}{$\begin{array}{c}====== \\
\text { By } \\
====== \\
\text { Rep. } \\
======0\end{array}$} & \multicolumn{4}{|c|}{ 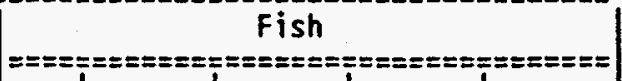 } \\
\hline & & \multirow{2}{*}{\multicolumn{4}{|c|}{ 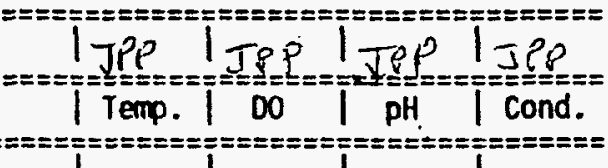 }} \\
\hline & & & & & \\
\hline & 1 & 244 & 5.7 & & \\
\hline$E-M E D$ & 2 & 246 & 5.6 & & \\
\hline Contral & 3 & 24.6 & 43 & 7.6 & \\
\hline \multirow[t]{2}{*}{$10 \pm 093,20.83$} & 4 & 247 & 5.9 & & 345 \\
\hline & 1 & 245 & 59 & & \\
\hline PCM 2.9 & 2 & 246 & 5.7 & & \\
\hline \multirow[t]{3}{*}{$50 \%$} & 3 & 24.2 & 62 & 7,9 & \\
\hline & 4 & 24.8 & 5.1 & & 364 \\
\hline & 1 & 246 & 5.7 & & \\
\hline PCM 2.9 & 2 & 24.8 & 5.5 & & \\
\hline \multirow[t]{3}{*}{$100 \%$} & 3 & 248 & 5.8 & 8.0 & \\
\hline & 4 & 24.6 & 5.5 & & 383 \\
\hline & 1 & 2448 & 5.4 & & \\
\hline $\operatorname{Pcm} 4.3$ & 2 & 243 & 46 & & \\
\hline \multirow[t]{3}{*}{$30 \%$} & 3 & $24 t$ & 48 & 2.7 & \\
\hline & 4 & $20 \%$ & 5.2 & & 371 \\
\hline & 1 & 248 & $4 k$ & & \\
\hline PCn 4.3 & 2 & $2+3$ & 5.2 & & \\
\hline \multirow[t]{3}{*}{$100 \%$} & 3 & 248 & 5.3 & 7.9 & \\
\hline & 4 & 24.7 & 5.0 & & 405 \\
\hline & 1 & 248 & 5.7 & & \\
\hline PC.M 5.1 & 2 & 24.4 & 5.3 & & \\
\hline \multirow[t]{3}{*}{$50 \%$} & 3 & 33.7 & $6 \cdot 2 x$ & 7,9 & \\
\hline & 4 & 24.5 & $4 i$ & & 377 \\
\hline & 1 & 24.4 & 56 & & \\
\hline PCM 5.1 & 2 & 34.4 & 58 & & \\
\hline \multirow[t]{2}{*}{15020} & 3 & 24.9 & 5.4 & 7.8 & \\
\hline & 4 & 24.8 & 5.7 & & 4,7 \\
\hline
\end{tabular}

Date $10-28$
Page 7 of 7

Personnel Simbeck Posey

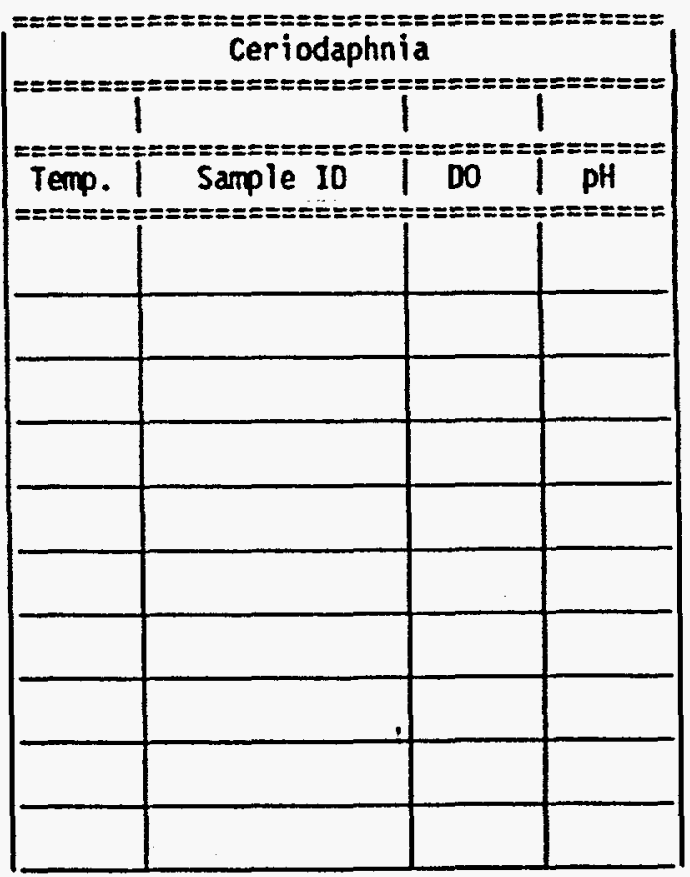

Reviewed By: $6+5$ 
Project/Study: CR-ERP Oct. 93

Personnel:
Posey
Beginning Date:

Ending Date:
HINKLER TITRATION METHOD

Alkal ine-lodide-Azide:

Brand Fisher

Lot $\# 910062-24$

Exp. 2.93

Sulfuric Acid:

Brand Fisher

Lot \#854507

Exp. NA

PH BUFFER SOLUTIONS

pH 4:

Brand Mallinckrodt

Lot \#0097 KLEA

Exp. $\frac{9-94}{9-1}$

$\mathrm{pH}$
Brand
Lot *
Exp.

Manganous Sulfate: Brand Fisher Lot \# $910067-24$

Exp. 4.93

Thyodene:

Brand Fisher

Lot \# 912254

Exp. NA

pH 7:

Brand Mallinackrast Lot 0098 KLKJ

Exp. $\frac{1-95}{1-25}$
Sodium Thiosulfate:

Brand RecCA

Lot \# $m 158$

Exp.
$\mathrm{pH} \cdot 10$ :

Brand Mallinckrodt Lot \# $0099 \quad<<B \beta$

Exp.

CONDUCTIVITY STANDARD SOLUTIONS

200 umhos:

Brand BIOPHARM

Lot \# 292.2

Exp.
720 umhos:

Brand Bro PHARM

Lot $\# C 303 B$

Exp. $4-95$ umhos:

Brand

Lot \#

Exp.

ALKALINITY TITRATION

Sulfuric Acid Solution N/50:

Brand Mall.nckiced

Lot \# H.36le

Exp. NA

MAROAESS TITRATION

Hardness Titrating Solution:

Brand Colain

Lot \# 22037700

Exp. NA

CHLORINE TITRATION

DPO Powder Pillows:

Brand $\frac{\mathrm{Hach}}{03 \mathrm{~cm}}$
Lot
Exp.
Hardness Indicator:

Brand Calgin

Lot \#DYIQB

Exp. $A L A$
Hardness Buffer Solution: Brand $\mathrm{Calgen}$ Lot $\# \frac{2606 B}{263}$

Exp.

FAS:

Brand $R_{K C A}$

Lot " Ho78

Exp. $\frac{11.4-93}{11.93}$ 
Project study CR-ERP Oct 193

Beginning Date $10-21-93$

Ending Date $10-28-93$

Do Meter

pH Keter(s)

Conductivity Meter

Thermometer(s)
Model 1's I Model 57

TVA Tag 531047

Calibration Date $2-18.93$

Model Drion Research Model 5A250

TVA Tag $8147+7413$

Calibration Date 4-30-93 $10 / 20193$

ModelOcion Research Model $399 A$

TVA Tag 543347

Calibration Date $5 \cdot 6-93$

Model YSI Model 32

TVA Tag 543369

Calibration Date $8 \cdot 12-93$

Model $\sum R T C O$

TVA Tag $M-148$

Calibration Date $12 \cdot 8 \cdot 92$

Model ERTCO

TVA Tag N1-253

Calibration Date 1,2.8.2.2 
ATTACHMENT III

CR-ERP AMBIENT WATER TOXICITY STUDY Reference Toxicant Test Information 
Crronle Reference Toxicant Tegt

Pathead MInnows - ARI

Test Date

$02 / 28 / 90$

$02 / 08 / 90$

$02 / 21 / 90$

$03 / 07 / 90$

$03 / 28 / 90$

$04 / 23 / 90$

$05 / 10 / 90$

$06 / 06 / 90$

$07 / 09 / 90$

$08 / 02 / 90$

$09 / 06 / 90$

$20 / 10 / 90$

$10 / 31 / 90$

$11 / 27 / 90$

$01 / 03 / 91$

$02 / 06 / 91$

$02 / 28 / 91$

$03 / 25 / 91$

$05 / 02 / 91$

$05 / 30 / 91$

$07 / 12 / 91$

$08 / 02 / 91$

$09 / 03 / 91$

10/02/91

$10 / 30 / 91$

12/02/91

$01 / 09 / 92$

$01 / 29 / 92$

$02 / 27 / 92$

$04 / 06 / 92$

$04 / 30 / 92$

$05 / 27 / 92$

$07 / 07 / 92$

$07 / 28 / 92$

$08 / 27 / 92$

$09 / 29 / 92 *$

$10 / 27 / 92$.

$12 / 01 / 92$.

$01 / 05 / 93$.

$01 / 26 / 93 *$

$02 / 24 / 93 *$

$03 / 23 / 93 *$

$04 / 26 / 93$.

06/01/93*

$07 / 06 / 93 * 1$

$08 / 04 / 93$.

$09 / 08 / 93$.

$09 / 30 / 93$.

$10 / 28 / 93$. survival

LOEC HOEC

$\begin{array}{ll}0.05 & 0.02 \\ 0.02 & 0.008 \\ 0.125 & 0.05 \\ 0.05 & 0.02 \\ 0.05 & 0.02 \\ 0.05 & 0.02 \\ 0.05 & 0.02 \\ 0.05 & 0.02 \\ 0.05 & 0.02 \\ 0.05 & 0.02 \\ 0.05 & 0.02 \\ 0.05 & 0.02\end{array}$

$0.02 \quad 0.008$

$0.05 \quad 0.02$

$0.05 \quad 0.02$

$0.125 \quad 0.05$

$0.05 \quad 0.02$

$0.05 \quad 0.02$

$0.02 \quad 0.008$

$0.02 \quad 0.008$

$0.05 \quad 0.02$

$0.05 \quad 0.02$

$0.05 \quad 0.02$

$0.02 \quad 0.008$

$<0.05 \quad 0.05$

$0.05 \quad 0.02$

0.050 .02

$0.05 \quad 0.02$

$0.05 \quad 0.02$

$0.02 \quad 0.008$

$0.02 \quad 0.008$

$0.05 \quad 0.02$

$0.02 \quad 0.008$

0.05 0.02

0.020 .008

0.020 .008

$0.02 \quad 0.008$

$0.05 \quad 0.02$

0.020 .008

$0.008 \quad 0.003$

0.020 .008

$0.05 \quad 0.02$

0.020 .008

$0.05 \quad 0.02$

$0.05 \quad 0.02$

$0.02 \quad 0.008$

$0.02 \quad 0.008$

$0.02 \quad 0.008$

$0.05 \quad 0.02$
Growth

LOEC

soec

$\begin{array}{ll}<0.008 & 0.008 \\ <0.008 & 0.008 \\ 0.02 & 0.008 \\ 0.008 & 0.003\end{array}$

$0.008 \quad 0.003$

$0.02 \quad 0.008$

$0.02 \quad 0.008$

$0.02 \quad 0.008$

$0.02 \quad 0.008$

$0.008 \quad 0.003$

$0.02 \quad 0.008$

$0.008 \quad 0.003$

$0.008 \quad 0.003$

$0.02 \quad 0.008$

$0.008 \quad 0.003$

$0.02 \quad 0.008$

$0.008 \quad 0.003$

$0.008 \quad 0.003$

$0.008 \quad 0.003$

$0.008 \quad 0.003$

$0.008 \quad 0.003$

$0.008 \quad 0.003$

$0.008 \quad 0.003$

$0.008 \quad 0.003$

$0.008 \quad 0.003$

$0.008 \quad 0.003$

$\begin{array}{lll}0.02 & 0.008 & 0.0109\end{array}$

$\begin{array}{llll}0.008 & 0.003 & 0.0089\end{array}$

$\begin{array}{lll}0.008 & 0.003 & 0.0067\end{array}$

$\begin{array}{llll}0.008 & 0.003 & 0.0059\end{array}$

$\begin{array}{lll}0.008 & 0.003 & 0.0085\end{array}$

$\begin{array}{lll}0.02 & 0.008 & 0.01\end{array}$

$\begin{array}{lll}0.008 & 0.003 & 0.0059\end{array}$

$\begin{array}{lll}0.008 & 0.003 & 0.0078\end{array}$

$\begin{array}{lll}0.008 & 0.003 & 0.0061\end{array}$

$\begin{array}{lll}0.008 & 0.003 & 0.0061\end{array}$

$\begin{array}{llll}0.008 & 0.003 & 0.006\end{array}$

$\begin{array}{lll}0.008 & 0.003 & 0.0068\end{array}$

$\begin{array}{lll}0.008 & 0.003 & 0.0056\end{array}$

$\begin{array}{llll}0.008 & 0.003 & 0.0039\end{array}$

$\begin{array}{llll}0.008 & 0.003 & 0.0052\end{array}$

$\begin{array}{lll}0.008 & 0.003 & 0.0056\end{array}$

$\begin{array}{llll}0.008 & 0.003 & 0.0055\end{array}$

$\begin{array}{llll}0.008 & 0.003 & 0.0074\end{array}$

$\begin{array}{lll}0.02 & 0.008 & 0.0119\end{array}$

$\begin{array}{llll}0.008 & 0.003 & 0.0054\end{array}$

$\begin{array}{llll}0.008 & 0.003 & 0.005 ?\end{array}$

$0.008 \quad 0.003 \quad 0.006$

$\begin{array}{lll}0.008 & 0.003 & 0.0049\end{array}$
Corrective Action Taken

+0.003 vas significant

- scarted using less than 24hr old fish

- flah were $24 h r$ a s3mina 
study lu Ref tox

Test organism Z Zatheacs

Beginning Date/Time $9 / 30 / 93$ lo5/5

Ending Date/Time $10.7-53 / 0830$

Personnel

Suler Posey

Control/Dilution Water 7 -ked

Test Treatment Identification

1. Contre stork Conc.
2. $\frac{0.001}{0.003}$
3. $\frac{0.008}{0.02}$
5. $\quad 0.05$
7.
8.

Spawn Date

$9 / 25 / 93$

Tile

$12,16,15,14$

$20,23,24,25$

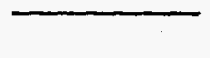

西 
ARL CHRONIC tOXICITY test MASTER GheckLISt study: Ca lef Toy Date Issued: $9-30.93$ To: Sesla

From: Moser

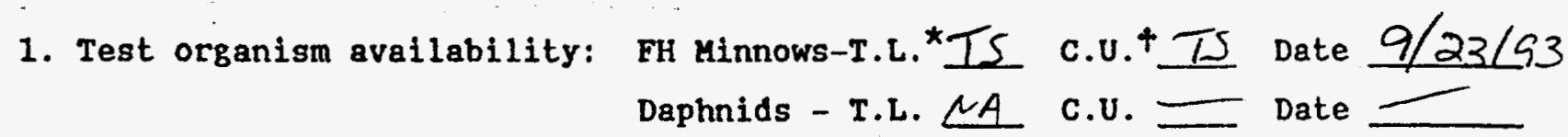

2. Sample Collection Coordination: Date $N A$ By $\longrightarrow$ with

3. Glassware availability: T.L. IS Tech. $\longrightarrow$ Date $9 / 29 / \sqrt{ } 3$

4. Glassware prep. complete: Date $9199 / 93$ By TS

5. Data sheet prep. complete: Date $9 / 29 / 53$ вy TS

6. Test organism availability verification: FH-OK? IS Daphnids-OK?

7. FH Minnow acclimation:

Day 1
By $\quad \angle 24$ m. oed

8. Test Initiation: FH-Date/Time 9/30/93: 0915 Daphnid-Date/Time Initial chem. TS FH TS Daphnid Log Samples

9. Test Renewal: Day \#: 1

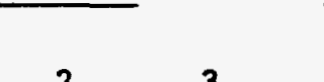

Initial Chem. - T.L. IS IS IS IS TS TS NA

T.K. *

Final Chem.

T.1.
F.H.
F.

T.M. JP

$T \frac{T S}{T P}$

TS

$\bar{r}$ Log Samples

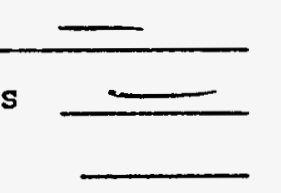

Daphnid

- T.L. IS IS IS IS IS IS IP IP IS

- T.L. wia N/A N/A NiA N/A N/A N/A

T.M. WIA NISt NIA WIA N/A N/A N/A

(8) 
study luReftox

FATHEAD MINNOW LARVAL SURVIVAL AND GROWTH TESI

Beginning Date/time 9/30/93:0915 Ending Date/Time 10-7-93/0830

Personnel

Page 1 of

sester Number Alive

\begin{tabular}{|c|c|c|c|c|c|c|c|c|}
\hline \multirow{2}{*}{ Treatment } & \multirow{2}{*}{ Rep } & \multicolumn{7}{|c|}{$-D a y-$} \\
\hline & & 1 & 2 & 3 & 4 & 5 & 6 & 1 \\
\hline \multirow[t]{5}{*}{ Control } & 1 & 10 & 10 & 10 & 10 & $1 ?$ & 10 & 10 \\
\hline & 2 & 10 & 10 & 10 & 10 & 10 & 10 & 10 \\
\hline & 3 & 10 & 10 & 10 & 10 & $B$ & 10 & 10 \\
\hline & 4 & 10 & 10 & 10 & iv & 10 & 10 & 12 \\
\hline & By: & $\pi$ & TS & IS & TS & is & IS & JP \\
\hline \multirow[t]{5}{*}{0.001} & 1 & 10 & 10 & 10 & 10 & 10 & 10 & 10 \\
\hline & 2 & 10 & 10 & 10 & 10 & 10 & 10 & 6 \\
\hline & 3 & io & 10 & 10 & 10 &, 0 & 10 & 10 \\
\hline & 4 & 10 & 10 & 10 & 10 & 10 & 10 & 10 \\
\hline & By: & $K_{1}^{\prime}$ & TS & $7 s$ & 75 & is & $T 3$ & $J P$ \\
\hline \multirow[t]{5}{*}{0.003} & 1 & 10 & 10 & 10 & 13 & 13 & 10 & 10 \\
\hline & 2 & 10 & 9 & 9 & 9 & 9 & 9 & 9 \\
\hline & 3 & 10 & 10 & 10 & 2 & 10 & 10 & 10 \\
\hline & 4 & 10 & 10 & 10 & 10 & 10 & 10 & 10 \\
\hline & By: & is & $I S$ & IS & IS & $I \leq$ & $T>$ & oms/ve \\
\hline \multirow[t]{5}{*}{0.008} & 1 & 10 & 10 & 10 & 2 & 10 & 10 & 10 \\
\hline & 2 & 10 & 10 & 10 & (i) & 10 & 9 & 9 \\
\hline & 3 & 10 & 10 & 10 & 10 & 10 & 9 & 9 \\
\hline & 4 & 10 & 10 & 10 & 10 & 10 & 10 & 10 \\
\hline & By: & Tskif & JP & $j p$ & JPtis: & $J P / D$ & $\partial f$ & $0 / 2$ \\
\hline \multirow[t]{5}{*}{0.02} & 1 & 710 & 10 & 10 & 10 & 10 & 9 & 8 \\
\hline & 2 & 10 & 10 & $i 0$ & 8 & 6 & 6 & 5 \\
\hline & 3 & 10 & ;0 & 10 & 8 & 7 & 6 & 6 \\
\hline & 4 & 10 & 10 & 10 & 9 & 8 & 8 & 7 \\
\hline & By: & $T P$ & $j p$ & $I P$ & $J P$ & $\overline{v P}$ & $\mathrm{IP}$ & $0<\alpha$ \\
\hline \multirow[t]{5}{*}{0.05} & 1 & 10 & 9 & 9 & $s$ & 6 & 6 & 6 \\
\hline & 2 & 10 & 10 & 10 & 8 & 7 & 6 & 5 \\
\hline & 3 & is & 10 & 8 & 6 & 5 & 3 & 3 \\
\hline & 4 & 10 & 10 & 10 & 8 & $6 s$ & $c$ & 5 \\
\hline & By: & To & $I P$ & IP & IP & $\overline{T P}$ & $J p$ & 418 \\
\hline
\end{tabular}

Notes:

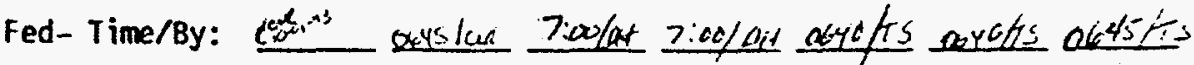

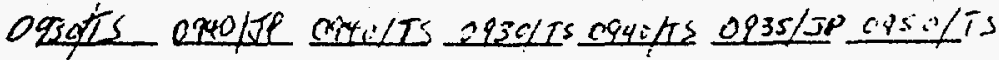

$1502 / T S$

$1500 / 751450 / 201500 / 3 p$

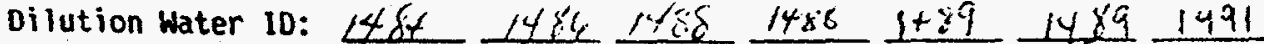

(PLARC501-257) Reviewed By: Is is is is is IP IP 
Analysis of Survival

REFTOX \#48

Starting Date: 09/30/93

\section{Survival (\%) Data}

-................

\begin{tabular}{|c|c|c|c|c|c|c|c|c|c|c|c|}
\hline \multirow[b]{2}{*}{ Treatment } & \multicolumn{11}{|c|}{ Replicate } \\
\hline & 1 & 2 & 3 & 4 & 5 & 6 & 7 & 8 & 9 & 10 & Mean \\
\hline CONTROL & 1.00 & 1.00 & 1.00 & 1.00 & . & . & . & • & . & - & 1.00 \\
\hline 0.001 & 1.00 & 1.00 & 1.00 & 1.00 & . & . & . & . & . & . & 1.00 \\
\hline 0.003 & 1.00 & 0.90 & 1.00 & 1.00 & . & . & . & - & . & . & 0.98 \\
\hline 0.008 & 1.00 & 0.90 & 0.90 & 1.00 & . & . & . & . & . & . & 0.95 \\
\hline 0.02 & 0.80 & 0.50 & 0.60 & 0.70 & . & - & 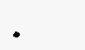 & - & - & . & 0.65 \\
\hline 0.05 & 0.60 & 0.50 & 0.30 & 0.50 & . & . & . & - & . & . & 0.48 \\
\hline
\end{tabular}

Steel's Many-One Rank Test

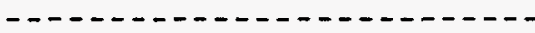

\begin{tabular}{lccc} 
Treatment & $\begin{array}{c}\text { No of } \\
\text { Replicates }\end{array}$ & $\begin{array}{c}\text { Critical } \\
\text { Rank Sum }\end{array}$ & Rank Sum* \\
\hline 0.001 & 4 & 10 & 18 \\
0.003 & 4 & 10 & 16 \\
0.008 & 4 & 10 & 14 \\
0.02 & 4 & 10 & 10
\end{tabular}

* Values less than Critical Rank Sum significantly less than the control (CONTROL). 
Non-paramatric analysis of toxicity data 2

4:04 Monday, October 14, 1991

STUDY=REFTOX \#48 ST_DATE=093093 PARM=MINS TEST=STEEL

CRITICAI SIGNIFICANT

OBS TREATMENT N CONTROL N EFFLUENT RANK SUM RANK SUM DIFFERENCE

$\begin{array}{lllll}0.001 & 4 & 4 & 18 & 10 \\ 0.003 & 4 & 4 & 16 & 10 \\ 0.008 & 4 & 4 & 14 & 10 \\ 0.02 & 4 & 4 & 10 & 10 \\ 0.05 & 4 & 4 & 10 & 10\end{array}$

10

10

10

10

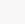


Non-paramatric analysis of toxicity data 1

4:04 Monday, October 14, 1991

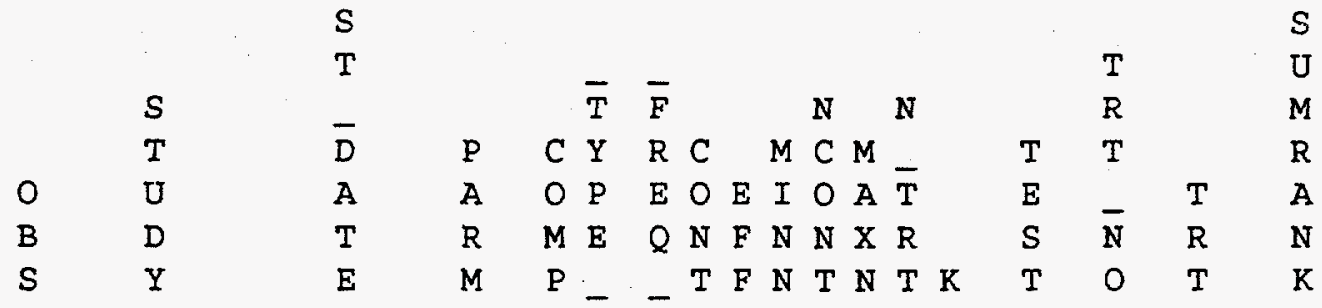

1 REFTOX \#48 093093 MINS 20010444444555 STEEL $10.001 \quad 18$

2 REFTOX \#48 093093 MINS 200104444444455 STEEL 20.00118

3 REFTOX \#48 093093 MINS $3 \quad 0 \quad 10444444455$ STEEL 10.00320

4 REFTOX \#48 093093 MINS $3 \quad 0 \quad 104444444456$ STEEL 30.00316

5 REFTOX \#48 093093 MINS 4 0 10444444445 STEEL $1 \quad 0.00822$

6 REFTOX \#48 093093 MINS 4 0 104444444456 STEEL 40.00814

7 REFTOX \#48 093093 MINS 5 0 $1044 \begin{array}{llllllllllll} & 4 & 4 & 4 & 5 & 5 & \text { STEEL } & 1 & 0.02 & 26\end{array}$

8 REFTOX \#48 093093 MINS 5 0 $104 \begin{array}{llllllllllll} & 4 & 4 & 4 & 4 & 5 & 5 & \text { STEEL } 5 & 0.02 & 10\end{array}$

9 REFTOX \#48 093093 MINS $6 \begin{array}{lllllllllllllll} & 0 & 10 & 4 & 4 & 4 & 4 & 4 & 5 & 5 & \text { STEEL } & 1 & 0.05 & 26\end{array}$

10 REFTOX \#48 093093 MINS $6 \begin{array}{llllllllllllllll} & 0 & 10 & 4 & 4 & 4 & 4 & 4 & 5 & 5 & \text { STEEL } & 6 & 0.05 & 10\end{array}$ 
- Tests of assumptions for survival data (arcsine transformed)

TEST FOR NORMAL DISTRIBUTION

4:01 Monday, October 14, 1991

STUDY $=$ REFTOX \#48 ST_DATE=093093

PARM $=$ MINS

UNIVARIATE PROCEDURE

Variable $=$ CENTER

Moments

$\begin{array}{lrlr}\text { N } & 24 & \text { Sum Wgts } & 24 \\ \text { Mean } & 0 & \text { Sum } & 0 \\ \text { Std Dev } & 0.081703 & \text { Variance } & 0.006675 \\ \text { Skewness } & -0.41677 & \text { Kurtosis } & 0.450456 \\ \text { USS } & 0.153532 & \text { CSS } & 0.153532 \\ \text { CV } & \text { Std Mean } & 0.016677 \\ \text { T:Mean=0 } & 0 & \text { Prob> }|T| & 1.0000 \\ \text { Sgn Rank } & 2 & \text { Prob> }|S| & 0.9299 \\ \text { Num ^ }=0 & 16 & & \\ \text { W: Normal } & 0.942557 & \text { Prob }<W & 0.1913 \\ & & & \\ & & & \\ & \text { Quantiles (Def }=5) & \\ 100 \% \text { Max } & 0.164704 & 99 \% & 0.164704 \\ 75 \% \text { Q3 } & 0.040743 & 95 \% & 0.126949 \\ 50 \% \text { Med } & 0 & 90 \% & 0.081485 \\ 25 \% \text { Q1 } & -0.02818 & 10 \% & -0.12223 \\ 0 \% \text { Min } & -0.17949 & 5 \% & -0.15705 \\ & & 1 \% & -0.17949 \\ \text { Range } & 0.344192 & & \\ Q 3-Q 1 & 0.068927 & & \\ \text { Mode } & 0 & & \end{array}$

Extremes

Lowest Obs Highest Obs

-0.17949 (

$-0.157051$

53) 0.048711(

44)

42) $0.081485(\quad 31)$

$-0.122231$

22) 0.081485(

34)

33) $0.126949($ 51)

$-0.081491$

32) 0.164704(

41)

$\begin{array}{lr}\text { Missing value } & . \dot{ } \\ \text { Count } & 36 \\ \% \text { Count/Nobs } & 60.00\end{array}$


- Tests of assumptions for survival data (arcsine transformed)

TEST FOR NORMAL DISTRIBUTION

4:01 Monday, October 14, 1991

\section{STUDY=REFTOX \#48 ST_DATE=093093 PARM=MINS}

UNIVARIATE PROCEDURE

Variable $=C E N T E R$

\begin{tabular}{|c|c|c|c|}
\hline Stem & Leaf & \# & Boxplot \\
\hline 1 & 6 & 1 & 0 \\
\hline 1 & 3 & 1 & 1 \\
\hline 0 & 588 & 3 & i \\
\hline 0 & 0000000033444 & 13 & +--+--+ \\
\hline-0 & & & +-- \\
\hline-0 & 886 & 3 & 1 \\
\hline-1 & 2 & 1 & 1 \\
\hline-1 & 86 & 2 & 0 \\
\hline
\end{tabular}

Multiply Stem. Leaf by $10 * *-1$

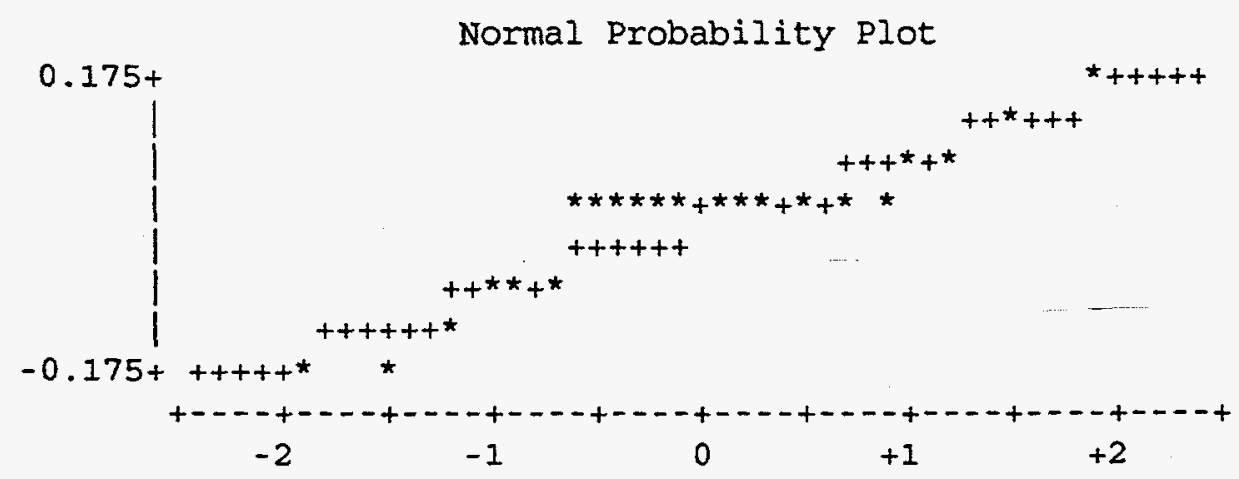


UNIVARIATE PROCEDURE

Schematic Plots

Variable $=$ CENTER

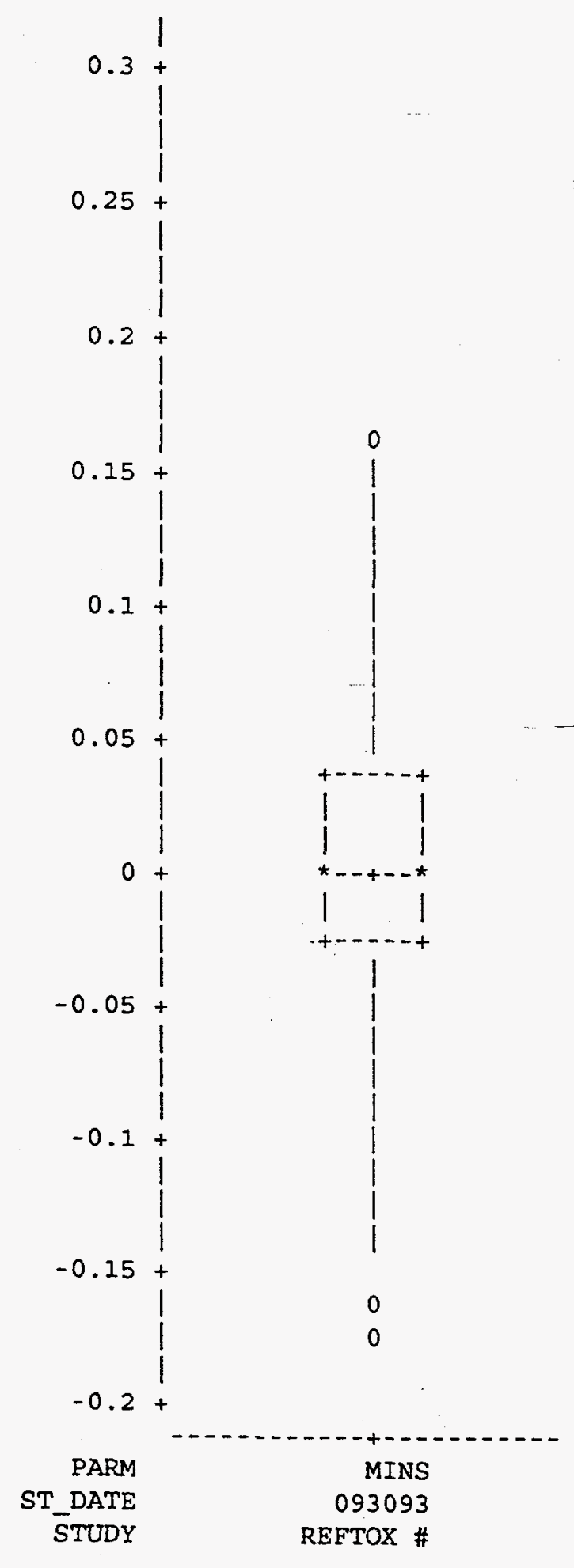


- Tests of assumptions for survival data (arcsine transformed)

TEST FOR NORMAL DISTRIBUTION

4:01 Monday, October 14, 1991

STUDY=REFTOX \#48 ST_DATE=093093 PARM=MINS

Plot of CENTER*RANKIT. Symbol is value of TRT_NO.

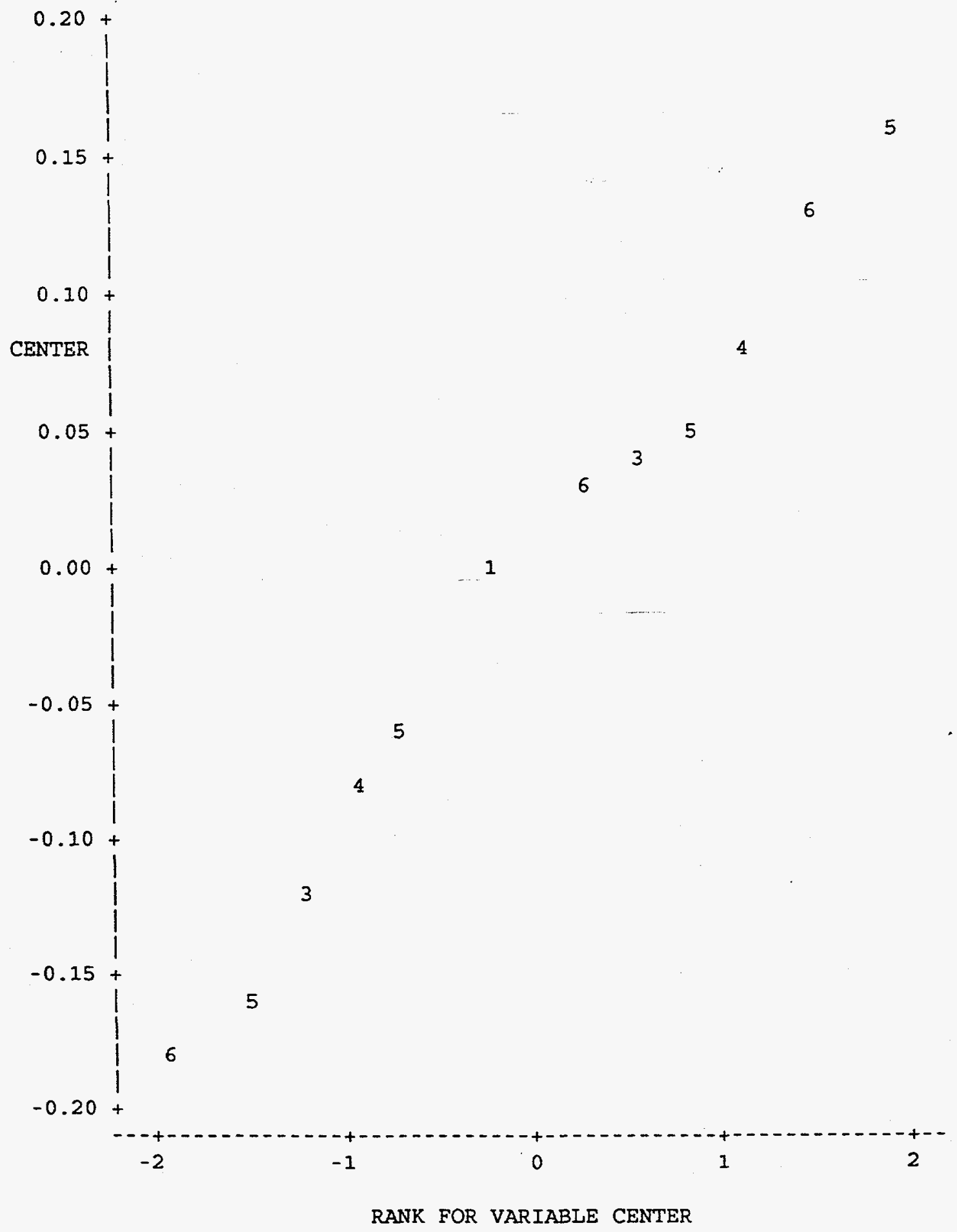

NOTE: 36 obs had missing values. 12 obs hidden. 


\section{FREQUENCY OF CENTER}

\section{FREQUENCY}

$8+$

7

7

6

5

4

$+$

1

$3+$

2

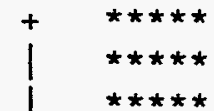

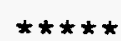

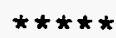

$1+$

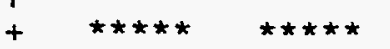

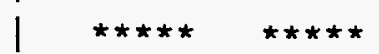

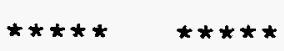

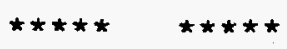

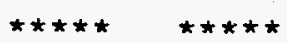

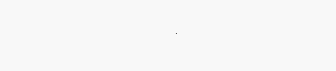

$\star \star \star \star \star ~$

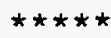

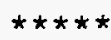

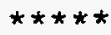

$\star \star \star \star * \star$

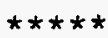

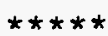

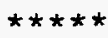

$\star \star \star * * *$

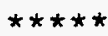

$\star \star \star * * *$

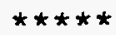

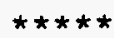

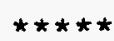

$\star \star \star \star \star ~$

$\star \star \star \star * *$

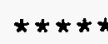

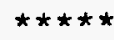

$\star \star \star * \star *$

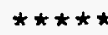

ॠ*t*

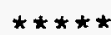

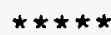

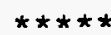

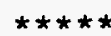

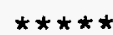

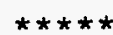

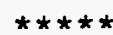

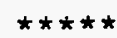

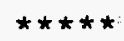

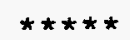

$\star \star \star \star * *$

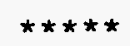

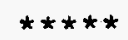

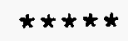

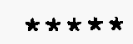

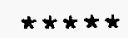

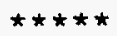

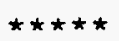

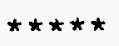

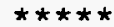

$\star \star * * *$

$\star \star \star \star \star ~ *$

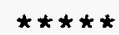

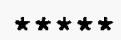

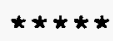

$\star \star \star \star * \star$

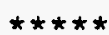

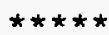

$\star * * * *$

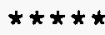

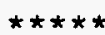

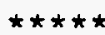

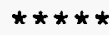

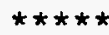

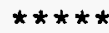

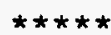

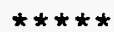

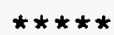

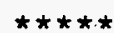

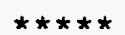

$\star \star \star \star \star$

$\star \star \star \star \star$

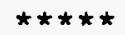

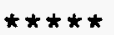

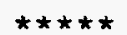

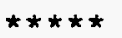

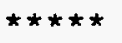

$\star \star \star \star \star$

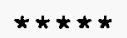

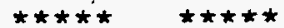

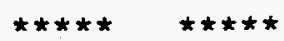

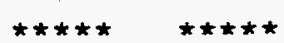

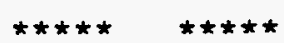

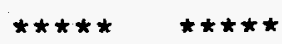

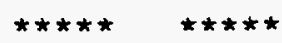

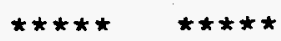

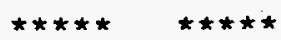

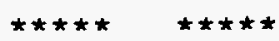

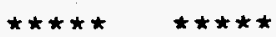
$-0.16$
$-0.12$
$-0.08$
$-0.04$
0.00
0.04
0.08
0.12

CENTER MIDPOINT 
TEST FOR NORMAL DISTRIBUTION

4:01 Monday, October 14, 1991

VARIANCE EQUAI, TO ZERO FOR ONE OR MORE TREATMENTS

BARTLETT'S. TEST CAN NOT BE CALCULATED

LEVENE'S TEST WILL BE USED 
- Tests of assumptions for survival data (arcsine transformed)

LEVENE'S TEST FOR HOMOGENEITY OF VARIANCE

4:01 Monday, October 14, 1991

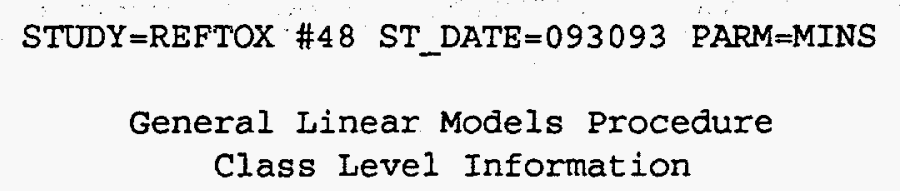

Class Levels Values

$\begin{array}{llllllll}\text { TRT } & 6 & 0.001 & 0.003 & 0.008 & 0.02 & 0.05 & \text { CONTROL }\end{array}$

Number of observations in by group $=60$

NOTE: Due to missing values, only 24 observations can be used in this analysis. 
: Tests of assumptions for survival data (arcsine transformed) LEVENE'S TEST FOR HOMOGENEITY OF VARIANCE

4:01 Monday, October 14, 1991

STUDY=REFTOX \#48 ST_DATE=093093 PARM=MINS

General Linear Models Procedure

Dependent Variable: ABS_VAL

\begin{tabular}{|c|c|c|c|c|c|c|}
\hline Source & DF & $\begin{array}{r}\text { Sum of } \\
\text { Squares }\end{array}$ & $\begin{array}{r}\text { Mean } \\
\text { Square }\end{array}$ & $F$ & Value & $\operatorname{Pr}>F$ \\
\hline Model & 5 & 0.04262409 & 0.00852482 & & 4.48 & 0.0079 \\
\hline Error & 18 & 0.03427130 & 0.00190396 & & & \\
\hline Corrected Total & 23 & 0.07689539 & & & & \\
\hline & R-Square & C.V. & RoOt MSE & \multicolumn{3}{|c|}{ ABS_VAL Mean } \\
\hline & 0.554313 & 77.21746 & 0.043634 & \multicolumn{3}{|c|}{0.05650847} \\
\hline Source & $\mathrm{DF}$ & Type I ss & Mean Square & F & value & $\operatorname{Pr}>F$ \\
\hline TRT & 5 & 0.04262409 & 0.00852482 & & 4.48 & 0.0079 \\
\hline Source & $\mathrm{DF}$ & Type III SS & Mean Square & $\mathbf{F}$ & Value & $\operatorname{Pr}>F$ \\
\hline RT & 5 & 0.04262409 & 0.00852482 & & 4.48 & 0.0079 \\
\hline
\end{tabular}


stady/oate Ces Reftor. Oet.93 Pans:Date/fine of Drylag $10 / 8 / 93 ; 10.55$ Pans:Date/rine of leighlog $10 / 8 / 93 ; 015$
PLTABAD DRT IBIGHT RBCORD SABBt

Page___ of 1

Personnel TCS

Pish:Date/tine of Orying $10 / 12 / 93 ; 1020$

Rish:Date/tine of Teighing $10 / 12 / 93 ; 1300$

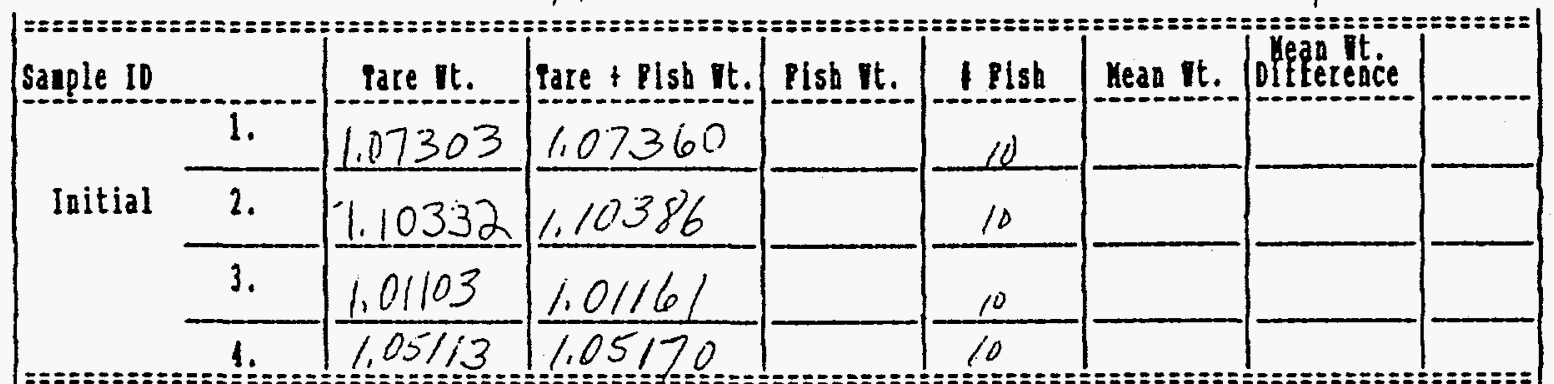

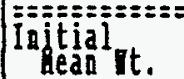

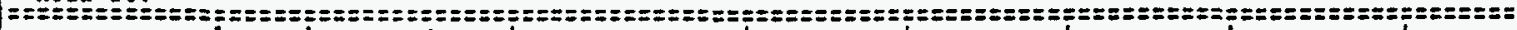

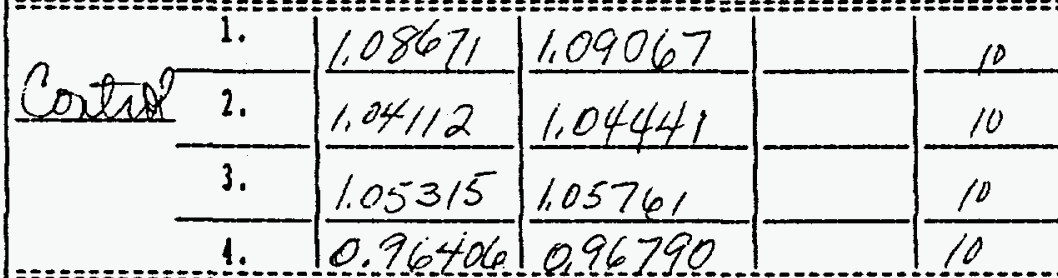

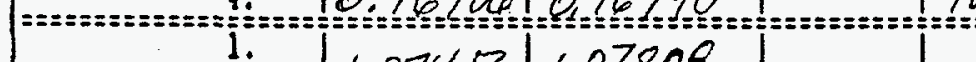
$\left|\frac{0.001 \frac{1 .}{1 .}}{\frac{1.07413}{3 .}}\right| \frac{1.07808}{\frac{1.12868}{1.0575}}\left|\frac{1.13311}{1.07324}\right| \frac{1.07782}{1 .}$ $\left|\frac{1.04645}{2.003}\right| \frac{1.05066}{2 .}\left|\frac{1.03103}{1.02828}\right| \frac{1.03562}{1.06435}\left|\frac{10323}{1.06922}\right|$

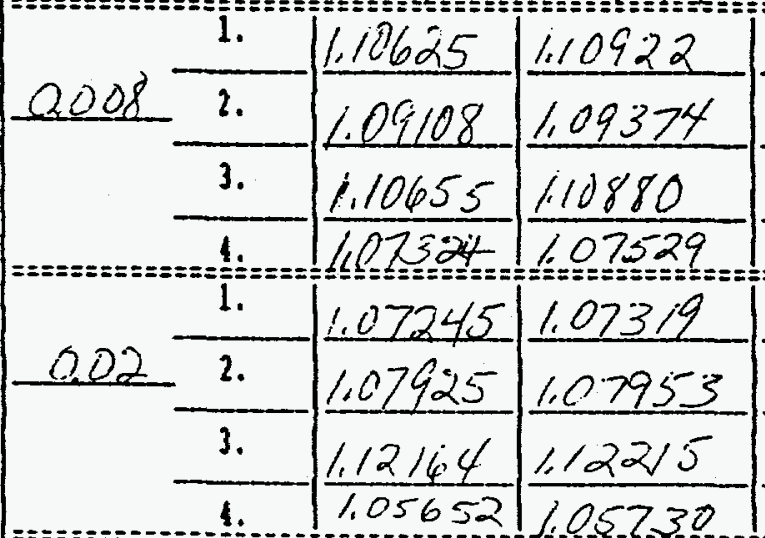
$\left|\frac{10}{10}\right|-\mid$

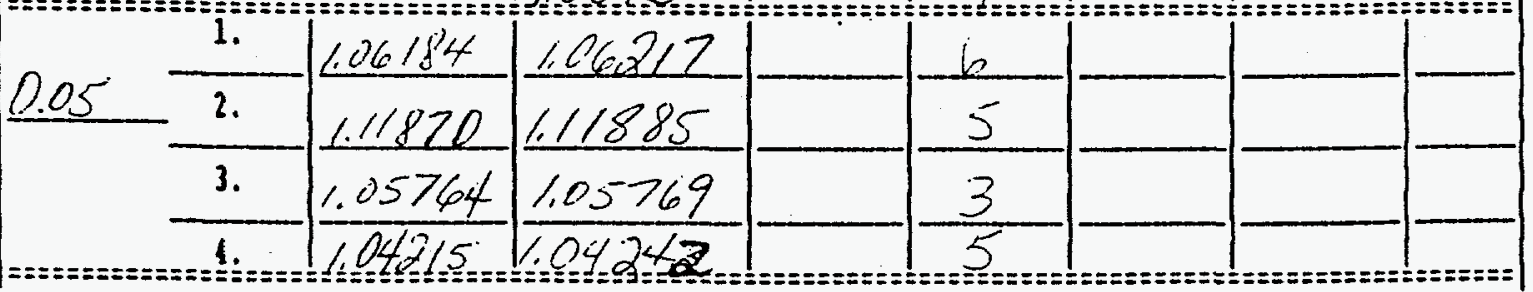


study/Date Gur Rej tox

Bas:Date/4ice of Orying $10 / 13 / 93 ; 0745$ Pish:Date/fine of Drying $10 / 13 / 93 ; 1010$

Pans:Date/fine of Ielghing 10/13/93; 0940

Pish:Date/line of Velghing $10 / 13 / 93 ; 1300$

Personnel I@S

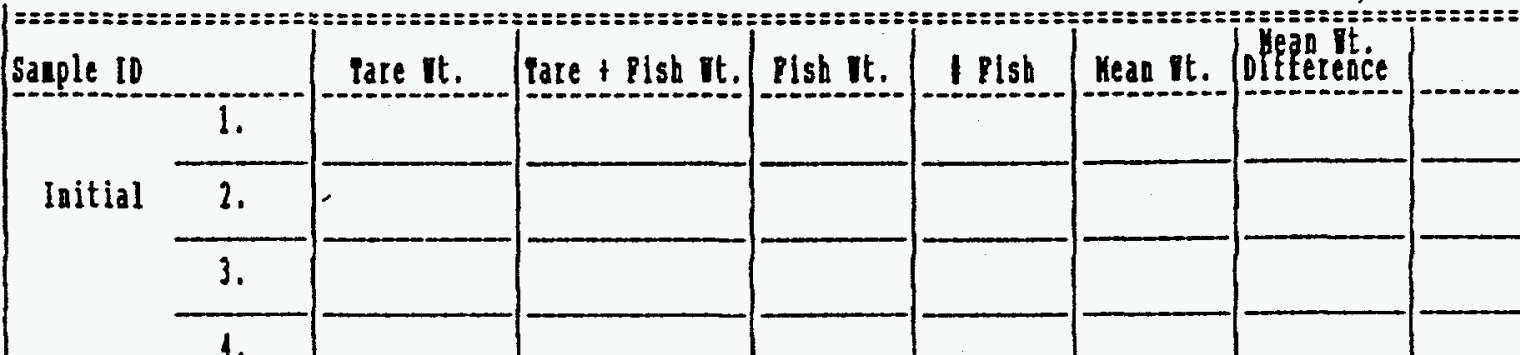

Initial

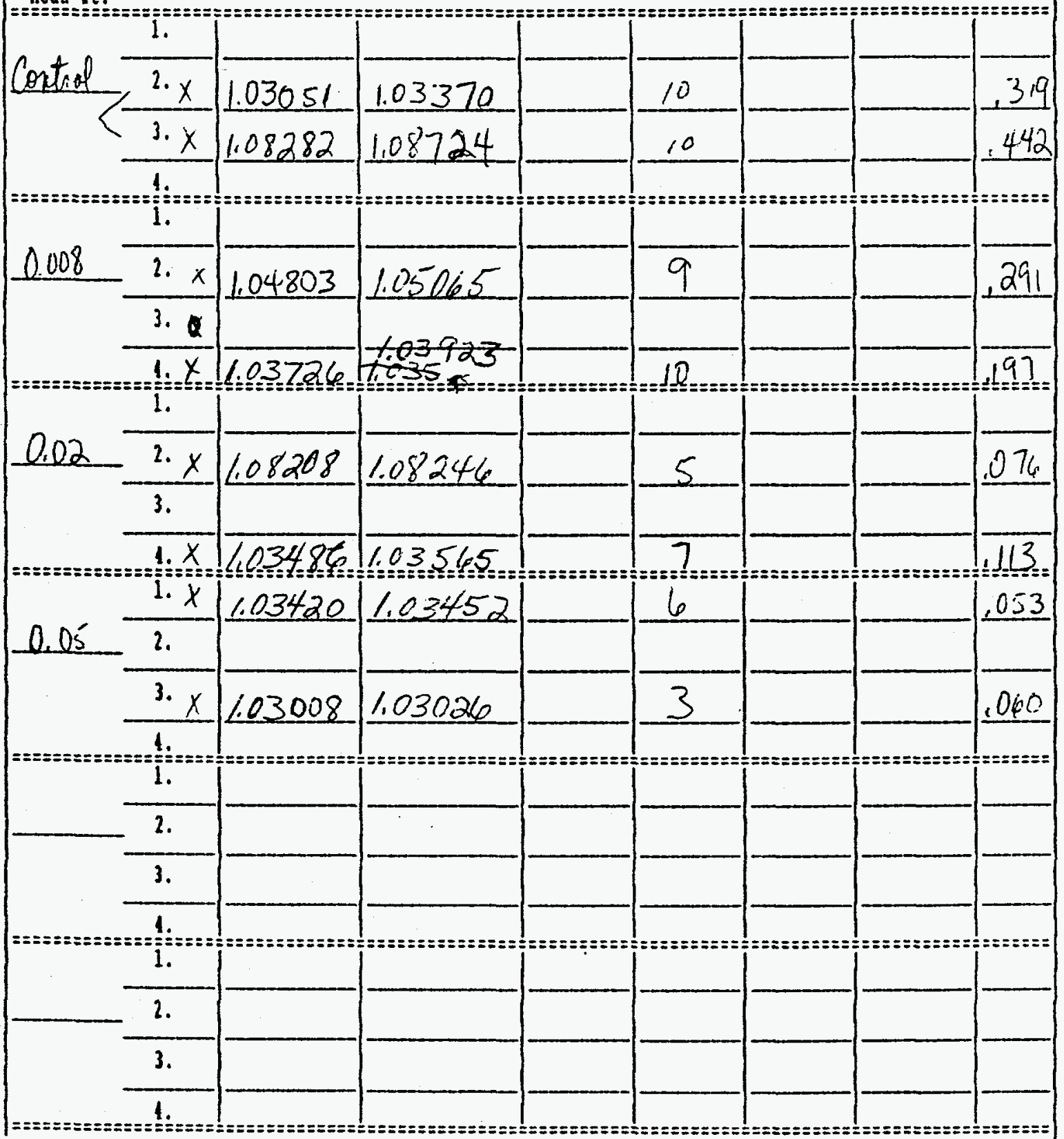

Revileved By: can 
Study/jate: Cu Ret Tox $\$ 48$

Dans:Deteline of Drying: $10 / 8 / 9 ?-1045$

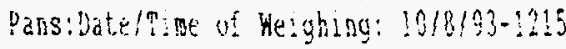

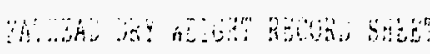

\section{useat in crealgais cer 10-2i-is}

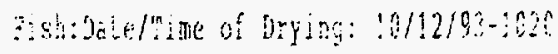

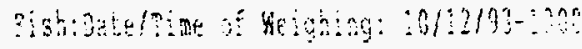

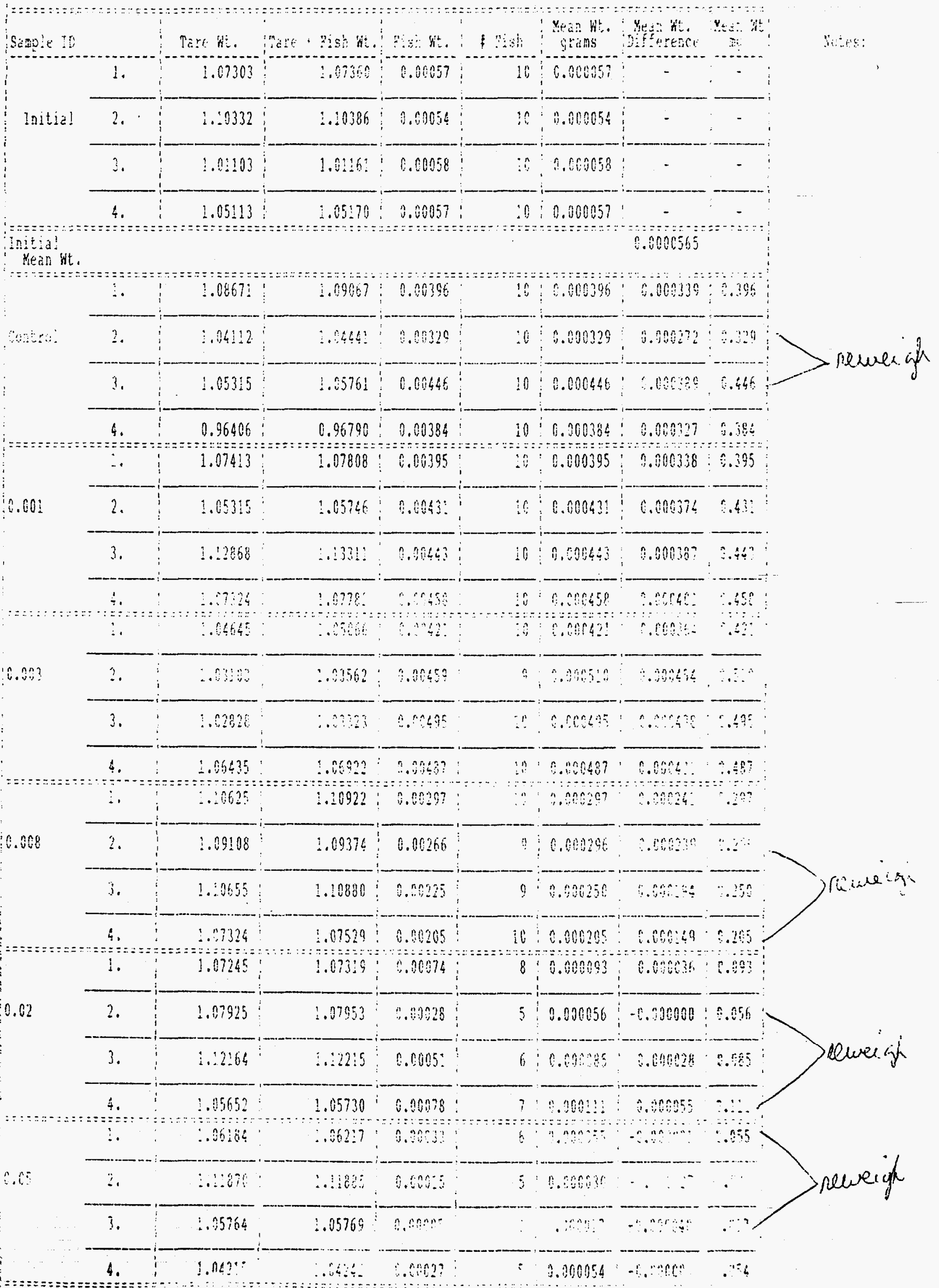




\author{
Analysis of 7-day Larval Growth Test \\ REFTOX \#48 \\ Starting Date: 09/30/93
}

\begin{abstract}
Analysis of Dry Weight (mg) Data Dunnett's Test
No transformation applied before data analysis.

For this set of data, the minimum significant difference is 0.07

This represents a $16.94 \%$ reduction in Dry Weight (mg).

$T=2.29$ ALPHA $=0.05$
\end{abstract}

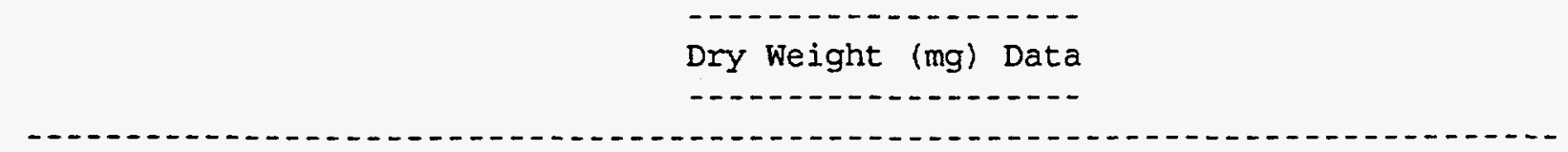

Replicate

\begin{tabular}{|c|c|c|c|c|c|c|c|c|c|c|c|}
\hline \multirow[b]{2}{*}{ TREATMENT } & & \multirow[b]{2}{*}{ MEAN } \\
\hline & 1 & 2 & 3 & 4 & 5 & 6 & 7 & 8 & 9 & 10 & \\
\hline 1 CONTROL & 0.40 & 0.33 & 0.45 & 0.38 & & & & & & & 0.39 \\
\hline 20.001 & 0.40 & 0.43 & 0.44 & 0.46 & • & $\bullet$ & . & • & . & . & 0.43 \\
\hline 30.003 & 0.42 & 0.51 & 0.50 & 0.49 & . & . & • & . & . & . & 0.48 \\
\hline 40.008 & 0.30 & 0.30 & 0.25 & 0.21 & . & . & . & . & . & . & 0.27 \\
\hline
\end{tabular}

Asterisk (*) indicates values significantly less than control (CONTROL).

\title{
Analysis of Variance
}

\begin{tabular}{lccccc} 
Source & DF & Sum OF Sq. & Mean Sq. & Calc $F$ & $F(0.05)$ \\
\hline Among & 3 & 0.102 & 0.034 & 20.440 & 0.0001 \\
Within & 12 & 0.020 & 0.002 & & \\
\hline Total & 15 & 0.000 & & & \\
\hline
\end{tabular}


Analysis of untransformed data

4:17 Monday, October 14, 1991

STUDY=REFTOX \#48

ST_DATE $=093093$

PARM=MING

DUNNETT'S TEST

$T=2.29$ ALPHA $=0.05$

TREATMENT

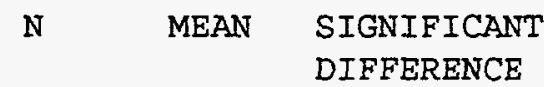

CONTROL

0.001

$4 \quad 0.39$

0.003

0.008

40.43

$4 \quad 0.48$

$4 \quad 0.27$

MINIMUM SIGNIFINANT DIFFERENCE $=0.07$

THIS REPRESENTS A $16.94 \%$ DECREASE IN SURVIVAL FROM THE CONTROL 
Analysis of untransformed data 2

4:17 Monday, October 14, 1991

STUDY=REFTOX \#48

ST_DATE $=093093$

PARM=MING

DUNNETT'S TEST

$T=2.29$ ALPHA $=0.05$

TREATMENT

REPLICATE

$\begin{array}{llllllllllll}1 & 2 & 3 & 4 & 5 & 6 & 7 & 8 & 9 & 10 & \text { MEAN } & S\end{array}$

1 CONTROL

$\begin{array}{llll}0.40 & 0.33 & 0.45 & 0.38\end{array}$

. . . $\quad .0 .39$

20.001

30.003

40.008

$\begin{array}{llll}0.40 & 0.43 & 0.44 & 0.46\end{array}$

$\begin{array}{lllll}0.42 & 0.51 & 0.50 & 0.49\end{array}$

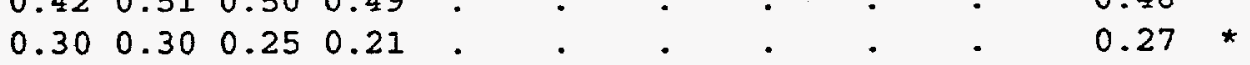

MINIMUM SIGNIFINANT DIFFERENCE $=0.07$

THIS REPRESENTS A $16.94 \%$ DECREASE IN SURVIVAI FROM THE CONTROL 
Analysis of untransformed data 3

$4: 17$ Monday, October 14, 1991

\section{General Linear Models Procedure}

Class Level Information

Class Levels Values

$\begin{array}{llllll}\text { TRT } & 4 & 0.001 & 0.003 & 0.008 \text { CONTROL }\end{array}$

Number of observations in data set $=40$

NOTE: Due to missing values, only 16 observations can be used in this analysis. 
Analysis of untransformed data
\[ 4: 17 \text { Monday, October 14, } 1991 \]

General Linear Models Procedure

Dependent Variable: RESP

\begin{tabular}{|c|c|c|c|c|c|c|}
\hline Source & $\mathrm{DF}$ & $\begin{array}{l}\text { Sum of } \\
\text { Squares }\end{array}$ & $\begin{array}{r}\text { Mean } \\
\text { Square }\end{array}$ & $F$ & Value & $\operatorname{Pr}>\mathrm{F}$ \\
\hline Model & 3 & 0.10206875 & 0.03402292 & & 20.44 & 0.0001 \\
\hline Error & 12 & 0.01997500 & 0.00166458 & & & \\
\hline \multirow[t]{3}{*}{ Corrected Total } & 15 & 0.12204375 & S & & & \\
\hline & R-Square & C.V. & Root MSE & & & RESP Mean \\
\hline & 0.836329 & 10.41131 & 0.0407993 & & & 0.39187500 \\
\hline Source & $\mathrm{DF}$ & Type I SS & Mean Square & $F$ & value & $\operatorname{Pr}>F$ \\
\hline TRT & 3 & 0.10206875 & 0.03402292 & & 20.44 & 0.0001 \\
\hline Source & $\mathrm{DF}$ & Type III SS & Mean Square & $\mathrm{F}$ & Value & $\mathrm{Pr}>\mathrm{F}$ \\
\hline TRT & 3 & 0.10206875 & 0.03402292 & & 20.44 & 0.0001 \\
\hline
\end{tabular}


Test for Normal Distribution

4:14 Monday, October 14, 1991

STUDY=REFTOX \#48 ST_DATE $=093093$ PARM=MING

UNIVARIATE PROCEDURE

Variable $=$ CENTER

Moments

\begin{tabular}{|c|c|c|c|}
\hline $\mathbf{N}$ & 16 & Sum wgts & 16 \\
\hline Mean & 0 & Sum & \\
\hline Sta Dev & 0.036492 & Variance & 0.001332 \\
\hline Skewness & -0.43449 & Kurtosis & -0.6875 \\
\hline USS & 0.019975 & CSS & 0.01997 \\
\hline CV & . & Std Mean & 0.009123 \\
\hline $\mathrm{T}:$ Mean $=0$ & 0 & Prob $>|\mathrm{T}|$ & 1.0000 \\
\hline Sgn Rank & 4 & Prob $>|s|$ & 0.84 \\
\hline $\operatorname{Num}^{\wedge}=0$ & 16 & & \\
\hline W:Normal & 0.936459 & Prob $<W$ & 0.30 \\
\hline \multicolumn{4}{|c|}{ Quantiles (Def $=5$ ) } \\
\hline $100 \% \operatorname{Max}$ & 0.06 & $99 \%$ & 0.06 \\
\hline $75 \%$ Q3 & 0.02875 & $95 \%$ & 0.06 \\
\hline $50 \% \mathrm{Med}$ & 0.00875 & $90 \%$ & 0.035 \\
\hline $25 \%$ Q1 & -0.02375 & $10 \%$ & -0.06 \\
\hline \multirow[t]{2}{*}{$0 \% \mathrm{Min}$} & -0.06 & $5 \%$ & -0.06 \\
\hline & & $1 \%$ & -0.06 \\
\hline Range & 0.12 & & \\
\hline$Q 3-Q 1$ & 0.0525 & & \\
\hline Mode & -0.06 & & \\
\hline
\end{tabular}

Extremes

$\begin{array}{rrrr}\text { Lowest } & \text { Obs } & \text { Highest } & \text { Obs } \\ -0.06( & 21) & 0.0275( & 14) \\ -0.06( & 2) & 0.03( & 22) \\ -0.055( & 34) & 0.035( & 31) \\ -0.0325( & 11) & 0.035( & 32) \\ -0.015( & 33) & 0.06( & 3)\end{array}$

Missing Value

Count

$\because$ Count/Nobs

60.00 
Tests of assumptions for untransformed data

Test for Normal Distribution

4:14 Monday, October 14, 1991

STUDY=REFTOX \#48 ST_DATE=093093 PARM=MING

UNIVARIATE PROCEDURE

Variable $=$ CENTER

$\begin{array}{rl}\text { Stem } & \text { Leaf } \\ 6 & 0 \\ 4 & \\ 2 & 08055 \\ 0 & 800 \\ -0 & 502 \\ -2 & 2 \\ -4 & 5 \\ -6 & 00\end{array}$

\#

1

5

3

3

1

1

2

Multiply stem. Leaf by $10 * \star-2$

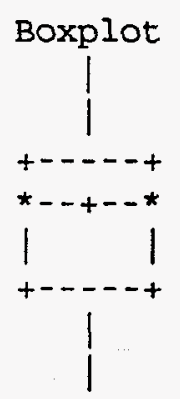




\section{UNIVARIATE PROCEDURE}

Schematic Plots

Variable $=$ CENTER

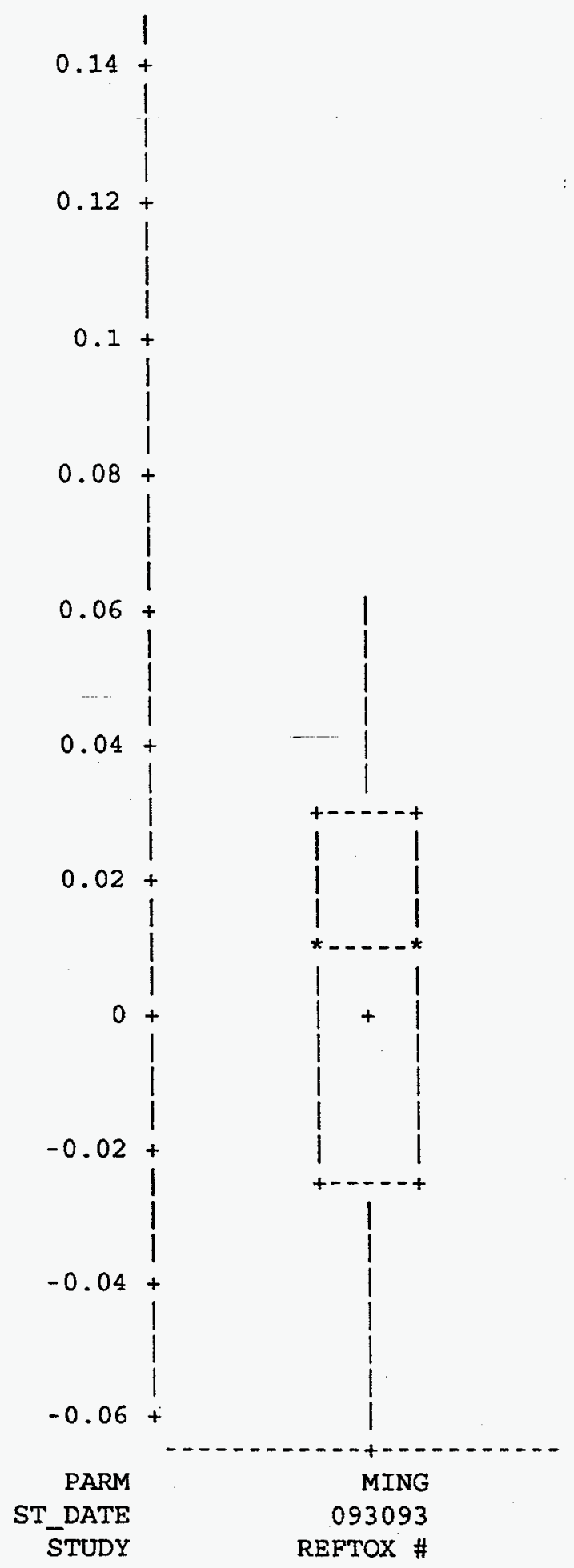


Plot of CENTER*RANKIT. Symbol is value of TRT_NO.

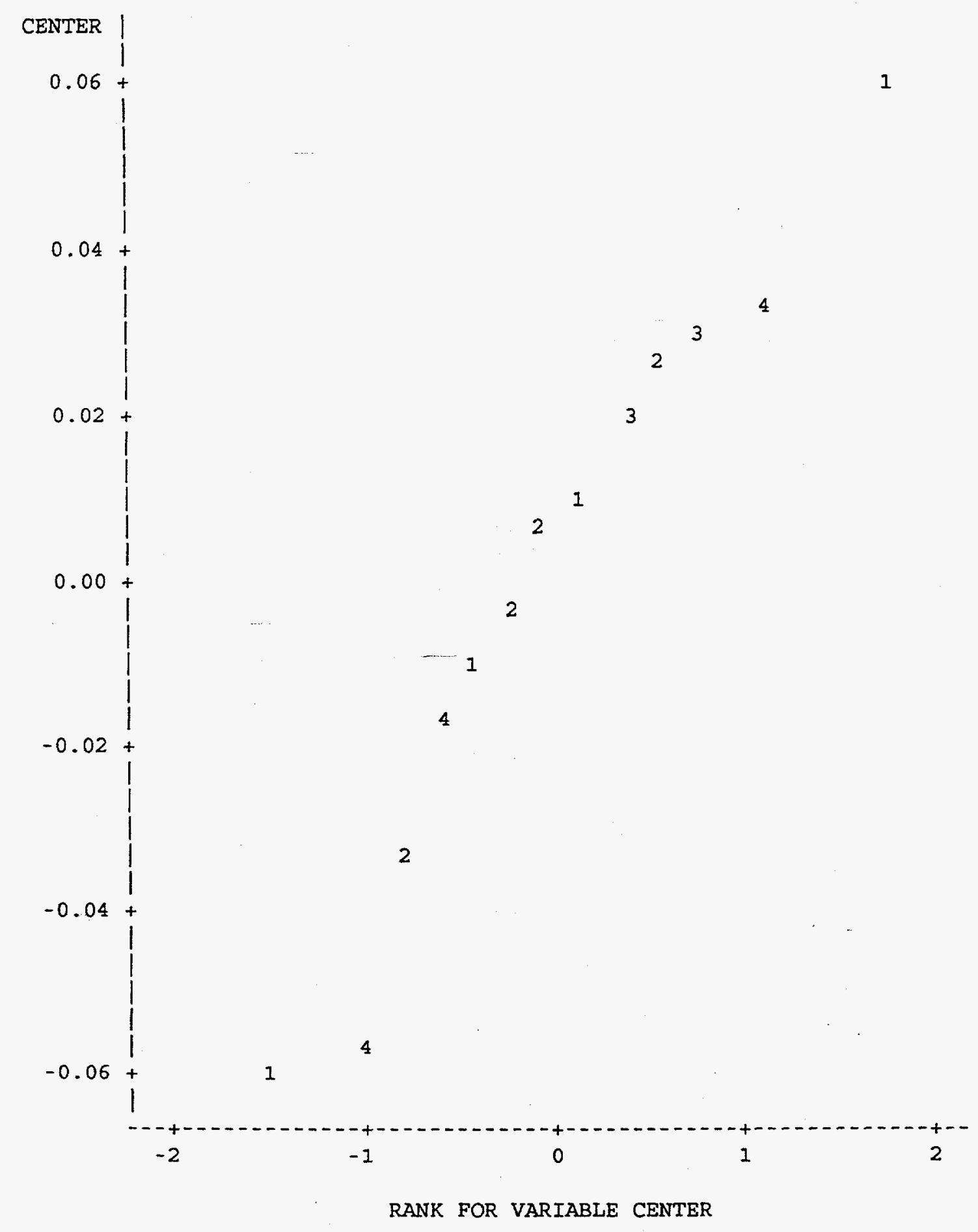

NOTE: 24 obs had missing values. 3 obs hidden. 
Tests of assumptions for untransformed data

Bartlett's Test for Homogeneous Variance

4:14 Monday, October 14, 1991

STUDY $=$ REFTOX \#48

ST_DATE $=093093$

PARM=MING

TREATMENT

$\mathbf{N}$

MEAN

VARIANCE

CONTROL

4

0.39

0.002

0.001

4

0.43

0.001

0.003

4

0.48

0.002

0.008

4

0.27

0.002

BARTLETT'S TEST STATISTIC $=1.193$

$\mathrm{PROB}>\mathrm{B}=0.755$ 
*** LISTING OF GROUP CONCENTRATIONS (\% EFF.) AND RESPONSE MEANS ***

$\begin{array}{ccc}\text { CONC. }(\% E F F) & \text { RESPONSE MEAN } & \text { MEAN AFTER POOLING } \\ .000 & .389 & .429 \\ .001 & .432 & .429 \\ .003 & .465 & .429 \\ .008 & .248 & .248 \\ .020 & .058 & .058 \\ .050 & .020 & .020\end{array}$

THE IINEAR INTERPOLATION ESTIMATE OF THE TOTAL IMPACT CONCENTRATION FROM THE INPUT SAMPLE IS .0060 .

.050

.020

.020

THE LINEAR INTERPOLATION ESTIMATE OF THE TOTAL IMPACT CONCENTRATION FROM THE INPUT SAMPLE IS .0060 .

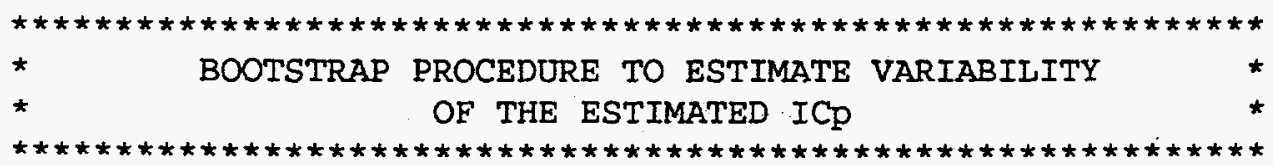

THE MEAN OF THE BOOTSTRAP ESTIMATES IS .0060.

THE STANDARD DEVIATION OF THE BOOTSTRAP ESTIMATES IS .0003 .

AN EMPIRICAL 95.0\% CONFIDENCE INTERVAL FOR THE BOOTSTRAP ESTIMATE IS $(.0055, .0067)$.

$C: \backslash B O O T S T R P\rangle$ 


$\begin{array}{lll}10 & .396 \\ 10 & .329 \\ 10 & .446 \\ 10 & .384 \\ 2.001 & .395 \\ 2.001 & .431 \\ 2 . .001 & .443 \\ 2.001 & .458 \\ 3.003 & .421 \\ 3.003 & .459 \\ 3.003 & .495 \\ 3.003 & .487 \\ 4.008 & .297 \\ 4.008 & .266 \\ 4.008 & .225 \\ 4.008 & .205 \\ 5.02 & .074 \\ 5.02 & .028 \\ 5 & .02 & .051 \\ 5 & .02 & .078 \\ 6.05 & .033 \\ 6.05 & .015 \\ 6.05 & .005 \\ 6.05 & .027\end{array}$

